\title{
Less is Different: Emergence and Reduction Reconciled
}

\author{
J. Butterfield \\ Trinity College, Cambridge University, Cambridge CB2 1TQ; email: jb56@cam.ac.uk
}

\begin{abstract}
This is a companion to another paper. Together they rebut two widespread philosophical doctrines about emergence. The first, and main, doctrine is that emergence is incompatible with reduction. The second is that emergence is supervenience; or more exactly, supervenience without reduction.

In the other paper, I develop these rebuttals in general terms, emphasising the second rebuttal. Here I discuss the situation in physics, emphasising the first rebuttal. I focus on limiting relations between theories and illustrate my claims with four examples, each of them a model or a framework for modelling, from well-established mathematics or physics.

I take emergence as behaviour that is novel and robust relative to some comparison class. I take reduction as, essentially, deduction. The main idea of my first rebuttal will be to perform the deduction after taking a limit of some parameter. Thus my first main claim will be that in my four examples (and many others), we can deduce a novel and robust behaviour, by taking the limit $N \rightarrow \infty$ of a parameter $N$.

But on the other hand, this does not show that that the $N=\infty$ limit is "physically real", as some authors have alleged. For my second main claim is that in these same examples, there is a weaker, yet still vivid, novel and robust behaviour that occurs before we get to the limit, i.e. for finite $N$. And it is this weaker behaviour which is physically real.

My examples are: the method of arbitrary functions (in probability theory); fractals (in geometry); superselection for infinite systems (in quantum theory); and phase transitions for infinite systems (in statistical mechanics).
\end{abstract}




\section{Contents}

1 Introduction

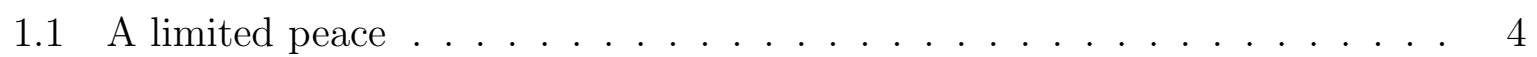

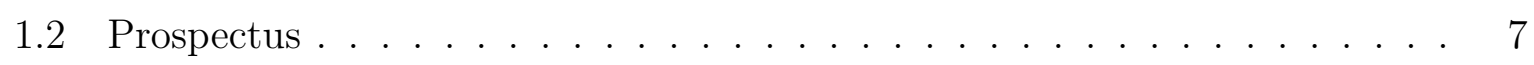

2 Becoming unrealistic on the way to the limit 9

3 Systems, states, quantities, values - and their limits 11

3.1 Emergence with and without infinite systems - and with ordinary limits . . 12

3.2 The limit of a sequence vs. what is true at that limit . . . . . . . . 13

3.3 Justifying $N=\infty \ldots \ldots \ldots \ldots$

3.3.1 Distinguishing straightforward from mysterious cases . . . . . . . 14

3.3.2 Dissolving the mystery . . . . . . . . . . . . . . . . . 16

3.3.3 Developing the Straightforward Justification . . . . . . . . . . . . . 19

4 The method of arbitrary functions $\quad 20$

4.1 Poincaré's legacy . . . . . . . . . . . . . . . . . . . . . . 21

4.1 .1 Poincaré's roulette wheel . . . . . . . . . . . . . 21 21

4.1.2 Generalizations: statistical stability . . . . . . . . . . 22

4.2 The claims illustrated by emergent equiprobability . . . . . . . . . 26

4.2.1 Emergence in the limit: with reduction - and without . . . . . . 26

4.2.2 Emergence before the limit . . . . . . . . . . . . . 27

4.2 .3 Supervenience is a red herring . . . . . . . . . . . . . 28

5 Fractals

5.1 Self-similarity and dimension as an exponent . . . . . . . . . . . . 29

5.1.1 Examples: scaling dimension . . . . . . . . . . . . . . . . 30

5.1.2 Generalizations: three other concepts of dimension . . . . . . . 32

5.2 The claims illustrated by emergent dimensions . . . . . . . . . . . 35

5.2.1 Emergence in the limit: with reduction - and without . . . . . 36

5.2 .2 Emergence before the limit . . . . . . . . . . . . . 37

5.2.3 Supervenience is a red herring . . . . . . . . . . . 38

5.3 The fractal geometry of nature? . . . . . . . . . . . . . . 39

5.4 The story so far: summing up fractals . . . . . . . . . . . . . . . 41 
6 Superselection 42

6.1 Introduction . . . . . . . . . . . . . . . . . . . . 42

6.1 .1 Out of the quantum soup $\ldots \ldots \ldots \ldots$

6.1.2 The idea of superselection in the limit . . . . . . . . 44

6.2 Superselection in the $N \rightarrow \infty$ limit of quantum mechanics . . . . . . 44

6.2 .1 Spin chains . . . . . . . . . . . . . . . . . 45

6.2.2 Continuous fields of algebras and deformation quantization . . . . 4 47

6.2.3 The classical infinite: macroscopic quantities from symmetric sequences . . . . . . . . . . . . . . . . . . . 49

6.2.4 The quantum infinite: quasi-local sequences . . . . . . . . . 52

6.2.5 Comparing the classical and quantum limits: classical states and the de Finetti theorem . . . . . . . . . . . . . . . . 55

6.3 The claims illustrated by emergent superselection . . . . . . . . . 55

6.3.1 Superselection from permutation-invariant states, in spin chains . . 55

6.3.2 Emergence in the limit: with reduction-and without . . . . . . 56

6.3 .3 Emergence before the limit . . . . . . . . . . . . 58

6.3 .4 Supervenience is a red herring . . . . . . . . . . . . 5 59

6.4 Summing up superselection . . . . . . . . . . . . . . 60 60

7 Phase transitions $\quad 60$

7.1 Phase transitions and thermodynamics $\ldots \ldots \ldots \ldots 6$. . . . . . . . . .

7.1.1 Separating issues and limiting scope . . . . . . . . . 6 61

7.1 .2 The thermodynamic limit . . . . . . . . . . . . . 6 62

7.2 The claims illustrated by emergent phase transitions . . . . . . . . 666

7.2.1 Emergence in the limit, and before it: Mainwood's proposal . . . . 67

7.2.2 Cross-over: gaining and losing emergence at finite $N \ldots \ldots 8$

7.3 Envoi . . . . . . . . . . . . . . . . . . . . . 70

8 References $\quad 70$ 


\section{Introduction}

\subsection{A limited peace}

'More is different!', proclaimed Philip Anderson in a famous paper (1972) advocating the autonomy of what are often called 'special' or 'higher-level' sciences or theories. A catchy slogan, indeed. But his reductionist opponents, such as Weinberg (1987), could have matched it, by invoking Mies van der Rohe's pithy defence of functionalist architecture: 'Less is more'. Hence my title. For my main point will be that although emergence is usually opposed to reduction, many examples exhibit both. So my title, 'Less is different', is meant as an irenic combination of the two parties' slogans. I will spell out this reconciliation in two claims, illustrated by four examples. The two claims, mnemonically labelled (1:Deduce) and (2:Before), are defined in Section 1.2 and each example is a model or a framework for modelling, from well-established mathematics or physics.

My irenic title is also ironic. For it deliberately echoes the sceptical refrain that there is nothing new under the Sun. Though I will not name names, most would agree that there is a good deal of heat, and rather less light, in the debate about emergence vs. reduction. Here's hoping that you will not recite that same refrain after reading this paper! Of course the heat and dark is in part due to different authors giving 'emergence' and 'reduction' different meanings. Thus I do not claim to be the only author to celebrate these words' compatibility. Among other celebrants, albeit using different meanings, are Simon (1996, pp. 249-251) and Wimsatt (1997, pp. 99-100 and references therein) 1

However, this is a companion to another paper (Butterfield 2010). So although the papers can be read independently, I should begin by describing their common aims and how they share out the work between them. In brief, both papers construe the contested terms, 'emergence' and 'reduction', as follows; (the other paper gives more details, and a defence of these construals; cf. its Sections 1.1, 2.1 and 3.1.1.).

I take emergence as behaviour that is novel and robust relative to some comparison class. In particular, my examples will be typical of many, by using two widespread conceptions of what the comparison class is, as follows. (1): Composites: The system is a composite; and its properties and behaviour are novel and robust compared to those of its component systems, especially its microscopic or even atomic components. (2): Limits: The system is a limit of a sequence of systems, typically as some parameter (in the theory of the systems) goes to infinity (or some other crucial value, often zero); and its properties and behaviour are novel and robust compared to those of systems described with a finite (respectively: non-zero) parameter. (Section 3 will explain how these ideas, (1) and (2), are better put in terms of quantities and their values, rather than systems.)

I take reduction as, essentially, deduction; though usually aided by appropriate definitions or bridge-principles linking the two theories' vocabularies. This will be close

\footnotetext{
${ }^{1}$ Other playful variations on Anderson's slogan occur in Kadanoff's splendid historico-philosophical introductions to phase transitions (2009, 2010, 2010a): which I will advert to in Section 7 . Cat (1998) is a scholarly review of the Anderson-Weinberg debate; Bouatta and Butterfield (2011) also contains a discussion.
} 
to endorsing the traditional account of Nagel (1961), despite various objections levelled against it. The picture is that the claims of some worse or less detailed (often earlier) theory can be deduced within a better or more detailed (often later) theory, once we adjoin to the latter appropriate definitions of the proprietary terms of the former. I also adopt a mnemonic notation, writing $T_{b}$ for the better, bottom or basic theory, and $T_{t}$ for the tainted, top or tangible theory; (where 'tangible' connotes restriction to the observable, i.e. less detail). So the picture is, with $D$ standing for the definitions: $T_{b} \& D \Rightarrow T_{t}$. In logicians' jargon: $T_{t}$ is a definitional extension of $T_{b}$.

In both papers, especially the other one, I consider a notion much discussed in the philosophy (but not physics) literature: supervenience (also known as 'determination' or 'implicit definability'). This is a less contested term. It is taken by all to be a relation between families of properties: the extensions of all the properties in one family relative to a given domain of objects determine the extension of each property in the other family. Besides, under wide conditions, this is a weakening of the usual notion of the second family being definable from the first, which is called 'explicit definability'. Roughly speaking, this weakening allows a definition of a property $P$ in the second family, in terms of the first family, to be infinitely long, rather than finite.

Since the definitions used in a Nagelian reduction are finite, supervenience is widely taken to be a weakening of Nagelian reduction. Besides, various philosophers have considered the infinity of "ways to be $P$ " given by an infinitely long definition to be a good way of making precise the heterogeneity or multiplicity of realization that philosophers have often associated with emergence. Thus arose the doctrine that emergence is "mere supervenience", i.e. supervenience without all the definitions being finite, as in a Nagelian reduction.

With these construals of the terms, the papers aim to rebut two widespread doctrines about emergence: the doctrine just mentioned, that emergence is mere supervenience, found in the philosophy literature; and the more widespread doctrine, found also in the physics literature (including the Anderson-Weinberg debate), that emergence is incompatible with reduction.

In the other paper, I develop these rebuttals in general terms; including a discussion of some other possible construals of the contested terms. I also emphasise supervenience, and thereby the first rebuttal. Thus I give (i) examples of mere supervenience which are not emergence and (ii) examples of emergence which are not mere supervenience nor reduction.

But in this paper, I will discuss the situation in physics and down-play supervenience, thus emphasising the second rebuttal. That is, I will argue that emergence is compatible with reduction, since physics gives examples combining both. The main idea will be to perform the reduction, i.e. deduction, after taking a limit of some parameter. Thus my first main claim, (1:Deduce), will be that in my four examples (and many others), we can deduce a novel and robust behaviour, by taking the limit $N \rightarrow \infty$ of a parameter $N$.

But on the other hand, this does not show that that the $N=\infty$ limit is "physically real", as some authors have alleged. For my second main claim, (2:Before), is that in 
these same examples, there is a weaker, yet still vivid, novel and robust behaviour that occurs before we get to the limit, i.e. for finite $N$. And it is this weaker behaviour which is physically real.

This contrast between strong and weak senses of emergence, and respectively its absence or presence at finite $N$, will be the main common theme across my four examples. It will also illuminate another current topic within philosophy of physics, about the significance of 'singular' limits in a physical theory. In fact, some authors propose to characterize emergence in terms of 'singular' limits.2 I deny this proposal. Although my two claims, and my four examples combining emergence and reduction, involve taking a limit, the limit is singular in only two of the four examples (the second and fourth, viz. fractals and phase transitions). So emergence is not always a matter of a singular limit-just as it is not always a matter of mere supervenience.

This negative verdict leaves open many questions, in particular: is emergence always a matter of a limit, whether singular or not? And even though a singular limit is not necessary for emergence, is it sufficient? In fact I think the answers to these questions are again 'No'. But I will not attempt to give a detailed characterization of emergence, whereby to prove these last two 'No's 3 The literature contains several such characterizations, with various merits. But as I explain in the other paper (especially Sections 1.1, 2.1), I doubt that there is - and that there needs to be - a single best meaning of 'emergence'; and similarly for 'reduction'. Anyway, I can develop my claims and examples while adopting my construals - of 'emergence' as novel and robust behaviour, and of reduction as deduction a la Nagel.

Before I give a prospectus (Section 1.2), I should make two final comments about these construals, and about my choice of examples. First: I submit that my construals of 'emergence' and 'reduction' are strong enough to make it worth exhibiting examples that combine them. Also, they seem to be in tension with each other: since logic teaches us that valid deduction gives no new "content", how can one ever deduce novel behaviour? This tension is also shown by the fact that many authors who take emergence to involve novel behaviour thereby take it to also involve irreducibility. The answer to the 'how?' question, i.e. my reconciliation, will lie in using limits: one performs the deduction after taking a limit of some parameter. So one main moral will be that in such a limit there can be novelty, compared with what obtains away from the limit.

Second: there is the issue of how I choose my examples. Here you may suspect what might be called the 'case-study gambit': trying to support a general conclusion by describing examples that have the required features, though in fact the examples are not

\footnotetext{
${ }^{2}$ I have used scare-quotes since writers often use the term loosely-too loosely, as I explain, and complain, in Section 3. But for easier reading, I will henceforth drop the scare-quotes.

${ }^{3}$ Incidentally, for the last 'No', that a singular limit is not sufficient for emergence: I agree with Wayne's argument for this (against Rueger (2000, p. 308; 2006, pp. 344-345)). Wayne uses Rueger's own example, of the van der Pol oscillator (Wayne 2009, Sections 3-5). My second example, fractals, will give another counterexample (Section 5.2.1): the topological dimension of a sequence of sets $C_{N}$ is discontinuous in the limit, i.e. $\lim _{N \rightarrow 0} \operatorname{dim} C_{N} \neq \operatorname{dim} \lim _{N \rightarrow 0} C_{N}$, but there is no emergence. For persuasive, more general, critiques of associating emergence or irreducibility with "singular asymptotics", cf. Belot (2005, especially Section 5) and Hooker (2004, pp. 446-458).
} 
typical, so that the attempt fails, i.e. the general conclusion, that all or most examples have the features, does not follow. But to this charge also I plead innocent, for the simple reason that I will not urge so general a conclusion, in the way that a reductionist opponent of Anderson might. (For example, I think Weinberg's objective reductionism (1987, p. 349-353) implies that (with my meanings of the terms) all known examples of emergence are also examples of reduction.) On the other hand, I do aspire to some generality! It will be clear that my claims, in particular my two main ones, (1:Deduce) and (2:Before), are illustrated by many examples beyond the four I have chosen. So I submit that the claims reflect the amazing power of Nagelian reduction.

\section{$1.2 \quad$ Prospectus}

Thus my main aim is to reconcile emergence with reduction, by arguing for two main claims, illustrated by four examples. Each example is a model, or a framework for modelling, from well-established mathematics or physics; and each involves an integer parameter $N=1,2, \ldots$ and its limit $N \rightarrow \infty$. In three of the examples, $N$ is, roughly speaking, the number of physical degrees of freedom of the system; in the second example, it is the number of iterations of a definitional process.

In all the examples, $N$ is, physically speaking, finite. But we can consider the limit: both what happens on the way to the limit, and what happens at it. (In Section 3, I will be more precise about the meaning of 'what happens', in terms of quantities being well-defined and what their values are.) Doing so yields my two main claims. The first is:

(1:Deduce): Emergence is compatible with reduction. And this is so, with a strong understanding both of 'emergence' (i.e. 'novel and robust behaviour') and of 'reduction' (viz. logicians' notion of definitional extension). In short: in the examples, considering $N \rightarrow \infty$ enables us to deduce novel and robust behaviour, in strong senses of 'novel' and 'robust'.

Besides, one needs to consider the limit in that: for each example, choosing a weaker theory using finite $N$ blocks the deduction of this strong sense. And (as discussed in Section 31), this weaker theory is appropriate and salient, i.e. liable to come to mind. Since the theories $T_{t}$ and $T_{b}$ are often defined only vaguely (by labels like 'thermodynamics' and 'statistical mechanics'), this swings-androundabouts situation explains away some of the controversy over whether $T_{t}$ is reducible to $T_{b}$.

The second claim is:

(2:Before): But on the other hand: emergence, in a weaker yet still vivid sense, occurs before we get to the limit. That is: in each example, one can understand 'novel and robust behaviour' weakly enough that it does occur for finite $N$.

Of my four examples, I have chosen the first three to be comparatively small, simple and agreed-upon, so that the philosophical issues stand out more clearly. They are from 
probability theory, geometry and quantum theory, respectively. The fourth example is an enormous topic in physics, with much less agreement. The examples are, in order:

1: The method of arbitrary functions, in probability theory; (Section 4);

2: Fractals, in geometry; (Section 5);

3: Superselection for infinite systems, in quantum theory; (Section 6);

4: Phase transitions for infinite systems, in classical statistical mechanics (Section 7).

Apart from the contrast between strong and weak senses of emergence shown by (1:Deduce) and (2:Before), there will be two other philosophical themes in common across the four examples.

The first is that supervenience is a "red herring", i.e. irrelevant. (So this supports the other paper's rebuttal of the doctrine that emergence is mere supervenience.) For clarity, it will again be best to give this a mnemonic label, as follows:

(3:Herring): Although various supervenience theses are true in the examples (and many others), the theses yield little or no insight - either into emergence, or more generally, into "what is going on" in the example.

We can already state the basic reason for this irrelevance. Supervenience allows that for each property $P$ in the "higher" i.e. supervening family of properties, there is, in the taxonomy given by the lower family, a disjunction of "ways to be $P$ ". But supervenience gives no "control" on this disjunction: not just in the sense that the disjunction might be infinite, but also that supervenience allows it to be utterly heterogeneous. In particular, no kind of limit is taken; and more generally, no connection is made between the variety, or infinity, of the disjunction and the limit processes, especially $N \rightarrow \infty$, which are crucial to the example. Thus supervenience is, at least in these examples (and, I submit, many others), too weak a concept to be enlightening.

The other theme in common across the four examples is that each example becomes, for finite but very large $N$, unrealistic in a vivid - one might even say: catastrophic - way. But this occurs for reasons external to current debates about emergence, reduction and the significance of limits of physical theories. It also will not undermine my (2:Before). This is because each of my examples illustrates (2:Before) for values of its parameter $N$ much smaller than those at which the example becomes unrealistic in the catastrophic way. So I will not emphasize this theme. On the other hand, the theme seems to have been completely neglected in these debates' literature; so it is worth spelling out. I will do this in Section 2, again giving it a mnemonic label, (4:Unreal).

After I discuss (4:Unreal), I give in Section 3 a general discussion of physical systems and their states and quantities, emphasizing the topic of limits: i.e. limits of systems, states and quantities, as some parameter $N$ (typically the number of degrees of freedom) goes to infinity. There are two related philosophical questions to be addressed. The first, mentioned in Section 1.1, is whether emergence can be characterized in terms of limits, especially singular limits. Contra some authors, I deny this; (along with others such as Wayne, Belot and Hooker).

The second question is whether in some examples, the singular limit is - not just indispensable for deducing emergence in some strong sense, or for epistemic concerns such 
as explanation and understanding-but also 'physically real'. These two questions are related in various ways: most obviously, by a Yes to the second implying that emergence according to the first would be physically real.

I shall also deny this: again, contra some authors. In more detail: my first claim, (1:Deduce), will illustrate how limits can be indispensable - viz. to deducing some novel and robust behaviour, where the behaviour in question is taken in a strong sense. And on the other hand, my second claim, (2:Before), will bring out how the $N=\infty$ limit is not physically real. That is: only the weaker sense of emergence that occurs at finite $N$ is physically real.

So let me sum up my claims. Emergence is not in all cases failure of reduction, even in the strong sense of reduction given by deduction (cf. (1:Deduce)). (Here, the deduction's need to invoke auxiliary definitions of the reduced theory's terms is made precise by logicians' notion of definitional extension; for details, cf. Section 3.1 of the companion paper). Nor does emergence in all cases occur only in the limit of the relevant parameter (cf. (2:Before)). Nor is emergence in all cases a matter of this limit being "singular" in some sense: my first and third examples will have non-singular limits (cf. also Section 3 ). Nor is emergence in all cases supervenience; nor is it in all cases failure of supervenience; (cf. Section 5 of the companion paper, and for the latter denial, (1:Deduce)). In short: we have before us a varied landscape - emergence is independent of these other notions.

\section{Becoming unrealistic on the way to the limit}

As we will see, my examples (and many other models, such as continuous models of fluids and solids) are examples of: formulating a formalism by taking an admittedly unrealistic limit of a parameter's value. But they are also examples of: formulating a formalism by taking a limit of a description which is admitted to be unrealistic on the way to the limit. This is my fourth labelled claim:

(4:Unreal): Each of the four examples becomes unrealistic before one gets to the $N=\infty$ limit - regardless of any technical issues about that limit, and regardless of any philosophical controversies about emergence.

One reason I need to discuss this claim is to show how it is consistent with (2:Before): the main point will be (as I mentioned) that (2:Before) applies to much smaller values of $N$. But phase transitions will also yield a remarkable illustration of "oscillations" between (2:Before) and (4:Unreal). In Section 7.2.2, we will see how a system can be manipulated so as to first illustrate (2:Before), i.e. an emergent behaviour at finite $N$, then lose this behaviour, i.e. illustrate (4:Unreal), and then enter a regime illustrating some other emergent behaviour (or revert to the first behaviour): a phenomenon called 'cross-over'.

There are also two other reasons why it is worth stating this claim, i.e. reasons unrelated to my own position in debates about emergence. First, almost all discussions

of emergence, or more generally of limiting relations between theories, in the physics and 
philosophy literatures, fail to notice this point. Agreed, some maestros notice it - though I do not mean to argue from authority! Thus Feynman: 'When you follow any of our physics too far, you find that you always get into some kind of trouble' (1964, Lecture 28.1) 4

Second, there is a common kind of reason for the un-realisticness ("break-down") of the examples. Besides, this kind of reason inevitably besets many other examples of taking limits of models as a parameter $N$, encoding physical degrees of freedom or some analogous concept, goes to $\infty$. So this commonality is worth registering, especially in discussions of emergence, or more generally of limits of models as a parameter $N \rightarrow \infty$.

In short, the commonality is: as $N$ becomes very large, the example runs up against either the micro-structure of space and its contents (for short: atomism), or the macrostructure of space and its contents (for short: cosmology). Thus my first two examples will run up against atomism: that is, very large $N$ will correspond to atomic or sub-atomic lengths, making what the example says utterly unrealistic. And my third and fourth examples will run up against cosmology: very large $N$ will correspond to cosmic lengths (and so gravity, and indeed spacetime curvature), again making what the example says utterly unrealistic. I stress that these break-downs are not internal to the model, but in relation to the actual world. To take my third and fourth examples: if there were no gravity nor spacetime curvature, and if space had the structure of $\mathbb{R}^{3}$, these examples, which postulate a chain of $N$ spins or a gas of $N$ molecules, in $\mathbb{R}^{3}$ without gravity, would indeed remain realistic as $N$ grows without bound.

I say 'in short', because in some examples Feynman's 'some kind of trouble' is not just either atomism or cosmology. The situation can be more varied. I will not enter into details, let alone try to classify the kinds of trouble. But to illustrate: my first example, the method of arbitrary functions, will include a model of a roulette wheel whose angular velocity tends to infinity; so the trouble will be, not atomism, but the fact that the model is Newtonian not relativistic! And more importantly: in my fourth example, phase transitions, some models run up against both atomism and cosmology. For in some models, the thermodynamic limit is not just the idea that keeping the density constant, the number $N$ of molecules (and so the volume) tends to infinity: there are also conditions on the limiting behaviour of short-range forces.

However, (4:Unreal) plays a different role in my discussion from my other three labelled claims: so I will not emphasize it as much as the others. There are two differences. First:

\footnotetext{
${ }^{4}$ I should mention another meaning of 'intermediate between small and infinite values of a parameter' that is noticed by the physics literature, under the label 'intermediate asymptotics': namely, a system's behaviour 'for times, and distances from boundaries, large enough for the influence of the fine details of the initial and/or boundary conditions to disappear, but small enough that the system is far from the ultimate equilibrium state' (Barenblatt 1996, p. xiii; cf. also p. 19). This meaning is obviously very different from this Section's 'intermediate $N$ ' regime. But it is worth mentioning, not just because of its intrinsic importance, but also because: (i) it is related to renormalization, which I will touch on in Section 7.2.2 (cf. also Goldenfeld et al. (1989)); and (ii) some philosophers (to their credit) have discussed it - though surely Batterman goes much too far when he writes 'I think, as should be obvious by now, that any investigation that remotely addresses a question related to understanding universal behavior [i.e. in philosophers' terms: multiple realizability] will involve intermediate asymptotics as understood by Barenblatt' (2002, p. 46).
} 
with one exception, discussions of these examples and many others - including discussions about emergence, and the examples' $N=\infty$ limit - do not, so far as I know, mention this un-realisticness for very large $N$. (The exception is my second example, viz. fractals.) Second: in each of my four examples, this un-realisticness for very large $N$ is not relevant to the ways that:

(1): a strong sense of emergent (i.e. novel and robust) behaviour can be deduced at the limit (cf. (1:Deduce)); and

(2): a weaker, yet still vivid, sense of emergent behaviour occurs on the way to the limit (cf. (2:Before)); and

(3): supervenience is a red herring, giving little or no insight into the example (cf. (3:Herring)).

So my discussion of emergence, in particular my main positive aim - the reconciliation got by combining my first two claims (1:Deduce) and (2:Before) - can proceed without discussing (4:Unreal). I stress again that each of the four examples illustrates (2:Before) for values of its parameter $N$ much smaller than those at which the example becomes unrealistic. (And this point applies in many other examples of taking limits of models as a degrees-of-freedom parameter goes to $\infty$.) So to keep the discussion of my examples as simple as possible, I will not explicitly refer there to (4:Unreal) - except at (i) the end of the second example, fractals, for which, as I said, the literature has noticed the point; and at (ii) the end of the fourth example, where the phenomenon of cross-over subtly combines (4:Unreal) with (2:Before).

\section{Systems, states, quantities, values - and their lim- its}

In Section 1.2, I promised that my two claims, (1:Deduce) and (2:Before), would clarify-I dare not say resolve! - the question whether in some examples of 'infinite' and-or 'singular' limits, the limit is not just epistemically indispensable but also 'physically real'. More specifically, I said I would agree about the indispensability, thanks to (1:Deduce), but deny the reality, thanks to (2:Before). But even before I show those claims in my examples, I can defend my general position; and in particular, justify my denying the physical reality of the limits. That is the job of this Section.

This is a job worth doing for two reasons. First, some discussions of emergence, and more generally, of limiting relations between theories are sloppy in their use of mathematical jargon about limits being 'singular' vs. 'regular/well-behaved/continuous' etc. And as I mentioned in Section 1.1, some authors even identify emergence with what happens at a 'singular' limit (Batterman (2002, pp. 6, 120, 127, 135), (2006, pp. 902-903), (2009, pp. 23-24); Rueger (2000, p. 308), (2006, pp. 344-345)). At least for my sense of emergence as novel and robust behaviour, this is wrong. In two of my four examples, there is nothing 'singular' about the limit. And recall that footnote 3 cited other arguments (and other authors) to the effect that a singular limit is not sufficient for emergence or irreducibility.

Second, some of the literature's physical examples and philosophical discussions are 
dauntingly complex. To take just one current philosopher: Batterman's examples include: (a) ray optics as a limit of wave optics; (b): classical mechanics as a limit of quantum mechanics; (c): hydrodynamics as a limit of molecular models; (d): phase transitions as described in the thermodynamic limit of statistical mechanics. Each of these is a large and complex area of physics, in which recent decades have seen a lot of deep and beautiful work - some of whose creators have themselves given masterly philosophical discussions (e.g. Berry 1994, Goldenfeld et al. 1989, Kadanoff 2009, 2010, 2010a). So there is a great deal for philosophers to address; (and all credit to Batterman and others for doing so). But we run the risk of being blinded by science, i.e. being misled by arcane technicalia. So I propose to discuss just one area, and even that only briefly: phase transitions, which will be my fourth example. (As I mentioned in Section 1.2, I chose my first three examples partly for their merit of being comparatively small and simple, so that the philosophical issues are clearer.) There is also a mountain of previous philosophical discussion, far too large to be addressed here. For apart from current authors like Batterman and Rueger, limiting relations between physical theories (singular or not) have long been a topic for authors such as Post, Schaffner, Scheibe, Rohrlich and Redhead. So I propose here just to spell out the general situation, as I see it. That will be enough to indicate how (at least in my examples!) there is no reason to believe the limit is physically real - a verdict which my examples will then confirm.

I divide the task in three subsections, 3.1 to 3.3. Sections 3.1 and 3.2 lay out some distinctions. Then Section 3.3 addresses the philosophical issue of what justifies our using a description with $N=\infty$. There I argue that even when the relevant limits are singular, a straightforward and broadly instrumentalist justification, viz. mathematical convenience and empirical correctness, applies: so that we need not believe the limit is physically real.

\subsection{Emergence with and without infinite systems - and with or- dinary limits}

We begin by envisaging physical systems, $\sigma$ say, each labelled by its parameter $N$, and thus a sequence of ever larger systems $\sigma(N)$. In all that follows (including my examples) $N \in \mathbf{N}:=$ the set of natural numbers. But nothing in this Section or the sequel depends on this: we could have $N \in \mathbb{R}$. We need to distinguish three questions, about systems, quantities and values respectively.

(1): One can ask whether this sequence has as a limit, in the sense of there being (as a mathematical entity) a natural well-defined infinite system $\sigma(\infty)$.

(2): One can ask whether a sequence of quantities on successive systems, say $f(N):=$ $f(\sigma(N))$, has a limit, which we might denote by $f(\infty)$. (Of course, the physical idea of each member of such a sequence will be in common, e.g. energy or momentum: but we distinguish the members by their being quantities on different (sizes of) system.)

(3): Finally, one can ask whether a sequence of real number values of quantities on successive systems, say $v(f(N)):=v(f(\sigma(N)))$, has a limit.

Of course, question (3) is the most familiar. The notion of limit is the elementary notion from calculus, $\lim _{N \rightarrow \infty} v(f(N))$. Here a sequence of states, $s_{N}$ say, on the $\sigma(N)$ is 
to be implicitly understood, so as to define values for the quantities $f(N)$; but to simplify notation, I will for the most part not mention $s_{N}$, and indeed take states as understood. Recall also from the calculus that if a real sequence $v_{N} \in \mathbb{R}$ grows without bound, i.e. for any number $M$ the $v_{N}$ eventually remain greater than $M$, we write: $\lim _{N \rightarrow \infty} v_{N}=\infty$. This is of course different from the idea (in Section 3.2 below) of taking $\infty$ as a possible value of the parameter or label on $v$, i.e. the idea of a sequence element $v_{\infty} \in \mathbb{R}$, which is after the denumerable sequence $v_{N}, N \in \mathbf{N} 5$

But we can also make sense of the first two questions. As to (1): in both classical and quantum physics we can often define the limit of a sequence $\sigma(N)$. Some approaches individuate a system by its state-space, and then use infinite cartesian or tensor products (for the classical and quantum cases respectively). Other approaches individuate a system by its set (in fact: algebra) of quantities, and then define limit algebras. This leads to how we make sense of (2). The algebra of quantities usually has a mathematical structure (in particular a topology) that enables one to define the limit of a sequence of quantities (i.e. not just, as in (1), a limit of a sequence of their values).

Note that the existence of an infinite system $\sigma(\infty)$ should not in general be identified with the existence of a limit quantity $f(\infty)$, or even several such; nor with the sequence of values $v(f(N))$ having a limit in the ordinary calculus sense. Indeed, my first example (the method of arbitrary functions) will illustrate this. There will be no infinite system $\sigma(\infty)$, but the sequences of values $v(f(N))$ will each have a limit in the ordinary sense - in fact a finite one, viz. $\frac{1}{2}$. These limits are in no way 'singular'. Yet there will be emergence, i.e. novel and robust behaviour.

There are also cases where there is (as a mathematical entity) an infinite system, and quantities defined on it whose values are the ordinary (in no way 'singular') limits of values on the finite systems; and where there is emergence. My third example (superselection in quantum theory) will illustrate this.

And finally there are cases that suit the enthusiastic talk about singular limits! That is: cases where there is (as a mathematical entity) an infinite system, and quantities defined on it that take "new" values, i.e. values different from the limits of values on the finite systems. My second and fourth examples (fractals and phase transitions) will illustrate this, the emergence being shown by these new values 6 Section 3.2 gives a few more details.

\subsection{The limit of a sequence vs. what is true at that limit}

The mathematical idea of this distinction is elementary. Recall that if we adjoin the number $\infty$ to the natural numbers $\mathbf{N}$, then we can consider sequences of real numbers $v_{n} \in \mathbb{R}$, with $n \in \mathbf{N} \cup\{\infty\}$, i.e. sequences of order-type $\omega+1$. For such sequences we can define the ordinary notion of limit, i.e. $\lim _{n \in \mathbf{N}} v_{n}$; and then of course we recognize that

\footnotetext{
${ }^{5} \mathrm{My}$ examples will of course need rather more calculus: e.g. we will need to distinguish between different kinds of convergence.

${ }^{6}$ But as argued in footnote 3. such discontinuous limits are not sufficient for emergence.
} 
there are cases in which $\lim v_{n}:=\lim _{n \in \mathbf{N}} v_{n}$ exists and is not equal to $v_{\infty}$. For $v_{\infty}$ means the $(\omega+1)$-th member of the sequence - a quite different idea from the ordinary limit!

Section 3.1 s idea of an infinite system $\sigma(\infty)$ allows us to apply this mathematical idea. We simply interpret adjoining the number $\infty$ to the set of finite values of $N$ as considering the infinite system $\sigma(\infty)$, as well as the finite systems $\sigma(N)$. I shall spell this out: first (a) for values of quantities, and then (b) for quantities themselves.

(a): Values of quantities: Suppose: (i) a sequence $v(f(N))$ of values of a quantity has a limit $\lim _{N \rightarrow \infty} v(f(N))$ as $N$ tends to infinity (as mentioned in Section 3.1, a sequence of states $s_{N}$ is here understood, so that one might write $\left.v\left(f(N), s_{N}\right)\right)$. And suppose also:

(ii) there is also a well-defined infinite system $\sigma(\infty)$ on which:

the common physical idea of the various $f(N)$ makes sense and gives a natural well-defined limit quantity, which we might write as $f(\sigma(\infty))($ on $\sigma(\infty))$; and on which there is a natural well-defined limit state, $s$ say.

Then we need to distinguish:

(i) the given limit of the values, $\lim _{N \rightarrow \infty} v(f(N)) \equiv \lim _{N \rightarrow \infty} v\left(f\left(N, s_{N}\right)\right)$, from

(ii) the value $v(f(\sigma(\infty), s)$ of the natural limit quantity $f(\sigma(\infty))$ in the natural limit state, $s$.

(b): Quantities: For quantities themselves, rather than values, the point is in essence the same. The statement is a close parallel of that in (a): indeed, shorter since we refer only to quantities, not to values of quantities - albeit thereby more abstract. Thus suppose: (i) a sequence of quantities $f(N)$ has a limit, dubbed $f(\infty)$ in Section 3.1. And suppose also: (ii) there is also a well-defined infinite system $\sigma(\infty)$ on which the common physical idea of the various $f(N)$ makes sense and gives a natural well-defined limit quantity, which we might write as $f(\sigma(\infty))$ (on $\sigma(\infty))$.

Then we need to distinguish:

(i) the given limit, $f(\infty):=\lim _{N \rightarrow \infty} f(N)$, from

(ii) the natural definition of the quantity $f(\sigma(\infty))$ on $\sigma(\infty)$.

\subsection{Justifying $N=\infty$}

\subsubsection{Distinguishing straightforward from mysterious cases}

'Justifying $N=\infty$ ' is of course a shorthand! For - to sum up Sections 3.1 and 3.2 -we have just learnt to distinguish two numbers: although in some models they are both welldefined and equal, they need not be! Namely:

(i): the $\operatorname{limit}_{N \rightarrow \infty} v(f(N))$ of a sequence of values (which limit might equal $\pm \infty$ );

(ii): the value $v(f(\sigma(\infty))$ of the natural limit quantity $f(\sigma(\infty))$ on the infinite system $\sigma(\infty)$.

So if we ask the question what justifies an " $N=\infty$ " model or description of a system, for which $N$ is actually finite, we must allow that the answers may be different for different models. (Here and in the rest of this Subsection, I consider, for simplicity, just values of quantities as in (a) of Section 3.2. not quantities themselves, as in (b) of Section 3.2.) 
We of course expect a straightforward justification for the two cases of 'non-singular' limits, i.e. the cases:

(a): (i) is well-defined (though perhaps $= \pm \infty$ ), but there is no infinite system so that (ii) is ill-defined; (cf. my first example, the method of arbitrary functions);

(b): there is an infinite system, and (i) and (ii) are both well-defined and are equal; (cf. my third example, superselection in quantum theory).

Namely, we expect a justification in terms of convenience and correctness, along the lines:

(Straightforward Justification): The use of the infinite limit-i.e. the use of (i) for case (a), and the use of (i) = (ii) for case (b) -is justified, despite $N$ being actually finite, by its being mathematically convenient and empirically correct (up to the required accuracy).

I shall develop and endorse this Justification in Section 3.3.3.

On the other hand, for 'singular limits', i.e. cases where (i) and (ii) are both welldefined but are not equal, and (ii) rather than (i) is empirically correct, matters are surely not straightforward. Such cases seem mysterious. Faced with such a case, should we give up the assumption that $N$ is actually finite? But in some examples, e.g. where $N$ is the number of molecules in a sample of gas (as in my fourth example, phase transitions), this apparently amounts to giving up the atomic constitution of matter 7

Nevertheless, some advocates of the philosophical importance of singular limits give up, or at least come very close to giving up, $N$ 's finiteness. I take as examples, three quotes from Batterman (his italics):

'a physically singular problem ... the "blow-ups" or divergences ... are the result of the singular nature of the physics' (2002, p. 56); 'real systems exhibit physical discontinuities ... genuine physical discontinuities - real singularities in the physical system' (2005, pp. 235-236); 'no de-idealizing story is possible even in principle' (2010, p. 17).

Agreed, in other passages, he holds back (thank goodness!):

'in (2005), I do speak rather sloppily of genuine physical singularities. It is best to think instead in terms of some kind of genuine qualitative change in the system at a given scale' (2010, p. 22); 'fluids are composed of a finite number of molecules' (2006, p. 903); 'water in real tea kettles consists of a finite number of molecules' (2010, p. 7; this quotation also occurs, together with its surrounding passage, at 2009, p. 9).

Note that this mysteriousness does not depend on (i) being well defined. If the $v(f(N))$ have no limit, not even $\pm \infty$, nevertheless the actual value is presumably $v\left(f\left(N_{0}\right)\right)$ for some actual but unknown $N_{0}$. So (ii) being empirically correct means that $v\left(f\left(N_{0}\right)\right) \approx$ $v(f(\sigma(\infty)))$ up to the required accuracy. But how can that be?

\footnotetext{
${ }^{7}$ Thanks to John Norton for stressing this point — as a reductio, of course.
} 


\subsubsection{Dissolving the mystery}

I think the mystery can be dissolved, in two stages. (1): First, I will concede that to deny that $N$ is finite might be a reasonable move. But in all the examples I know, in particular in all of my examples, this move is wrong. (Here, the important point is that in my second and fourth examples, fractals and phase transitions, this move is wrong: for as noted in Section 3.3.1, my first and third examples of emergence have no mysterious 'singular limit'.) So the more important stage will be the second one, (2) below: viz., that we need to consider quantities other than $f$. I turn to details.

(1): Denying that $N$ is finite; other degrees of freedom:- I admit it can be reasonable to deny that $N$ is finite. But this means something less radical than denying atomism! Rather we conclude that the finite- $N$ model has not picked the right, or not all the right, degrees of freedom for understanding the system; and that the (model of the) infinite system has somehow 'clued in to' the missing relevant degrees of freedom, as shown by its empirical correctness.

My fourth example, phase transitions in statistical mechanics, provides a putative example. Assuming that the correct description of a boiling kettle requires infinitely many degrees of freedom, it is reasonable to say that, since the kettle contains finitely many atoms, and so finitely many mechanical degrees of freedom, other degrees of freedome.g. of the electromagnetic field - must somehow be involved. Reasonable: but very programmatic! In fact, there is good evidence that the electromagnetic field is not involved in phase transitions - suggesting that the answer to the mystery lies elsewhere ...

(2): Other quantities:- The mystery is an artefact of focussing on just one quantity ( $f$ in my notation). Once we consider appropriate other quantities (and maybe related mathematical notions), the mystery dissolves. Thus in my second and fourth examples (fractals and phase transitions), there are other quantities, for which (despite $f$ 's singular limit) the finite- $N$ model, for large $N$, is close to the values given by the infinite model: and is thereby also empirically correct. In fact, these other quantities are 'cousins' of the quantity $f$ which we first considered. Thus the mystery will be dissolved by my second claim, (2:Before): namely, we see a weak yet vivid version of the emergent behaviour before we get to the limit. Besides, I would claim - though I cannot defend it in this paperthat this is so in all of physics' similar cases (in particular, in Batterman's examples from optics, semiclassical mechanics and hydrodynamics).

Agreed, for me to say 'there are other quantities or notions for which the finite- $N$ model is close to the infinite model' or 'we see a weak version of emergence before the limit', is unsatisfyingly abstract. Indeed, it is dismayingly close to the mysterious explanandum, viz. that the infinite model is empirically correct! But I submit that at this very general level, these formulations are the best one can do. To see vividly how the mystery dissolves, one has to look at examples; cf. my second and fourth examples. But here is a simple mathematical example illustrating the issues - and that there really is no mystery! As we shall see, it is not just a mathematical toy: it models physical situations, especially phase transitions. 
Consider the sequence of real functions $g_{N}: \mathbb{R} \rightarrow \mathbb{R}, N \in \mathbf{N}$, defined by

$$
\begin{array}{r}
g_{N}(x):=-1 \text { iff } x \leq \frac{-1}{N} ; \\
g_{N}(x):=N x \text { iff } \frac{-1}{N} \leq x \leq \frac{1}{N} ; \\
g_{N}(x):=+1 \text { iff } \frac{1}{N} \leq x .
\end{array}
$$

Thus $g_{N}(x)$ is constant and equal to -1 (respectively +1 ) for $x$ less than $\frac{-1}{N}$ (respectively: greater than $\left.\frac{1}{N}\right)$; and it increases linearly, with gradient $N$, over the interval $\left[\frac{-1}{N}, \frac{1}{N}\right]$, so that for all $N, g_{N}(0)=0$. Each $g_{N}$ is continuous; but the sequence has as its limit the function $g_{\infty}$ given by

$$
g_{\infty}(x)=-1 \text { iff } x<0 ; g_{\infty}(0)=0 ; g_{\infty}(x)=1 \text { iff } 0<x ;
$$

which is discontinuous at 08 So this limit is 'singular' in the sense that continuity is lost.

We can make this more formal by introducing a two-valued quantity $f_{N}, N \in \mathbf{N} \cup\{\infty\}$ that encodes whether or not $g_{N}$ is continuous: $f_{N}:=0$ if $g_{N}$ is continuous and $f_{N}:=1$ if $g_{N}$ is discontinuous. Then we have: $f_{N}=0$ for all finite $N \in \mathbf{N}$, but $f_{\infty}=1$. So in our (i)/(ii) notation (from Sections 3.2 and 3.3.1), we have a case where (i) and (ii) are both well-defined but are unequal.

But there is no mystery here! There only seems to be a mystery if we look solely at the two-valued quantity $f_{N}$, whose values report that the limit is 'singular', but which say nothing about how "close", for large $N$, the $g_{N}$ are to $g_{\infty}$.

Besides, there remains no mystery if we add some physical interpretation to the discussion. Thus imagine that the values of $g_{N}$ in a neighbourhood of 0 , or the slope of $g_{N}$ thereabouts, are part of a model of a system with $N$ degrees of freedom. $N$ varies, and is in general large; so that one considers the sequence of functions $g_{N}$. Now imagine that for large $N$, it is hard to know the actual value $N_{0}$ of $N$ and-or hard to calculate the value of $g_{N}$, even if you know $x$. (Agreed: my example is so simple that only a dimwit could find the calculation hard! Such is the price of a simple example ...) In this situation, it obviously could be both (a) mathematically convenient and (b) empirically accurate - i.e. close enough to the predictions made by $g_{N_{0}}(x)$ for the actual $x$ - to work with $g_{\infty}$.

For as to (a): $g_{\infty}$ 's being discontinuous need not make it inconvenient. Better the discontinuous $g_{\infty}$ that you can get a grip on, than the hard-to-know and-or hard-tocalculate $g_{N_{0}}$ ! And as to (b): as $N$ grows, the range of $x$ for which $g_{N}(x) \neq g_{\infty}(x)$ becomes arbitrarily small. Besides, for $x=0$ - which might be a physically significant argument $-g_{\infty}$ is completely accurate: i.e. for all $N, g_{N}(0)=g_{\infty}(0)$. In short: again, no mystery. There only seems to be a mystery if we look solely at $f_{N}$, and ignore the details about $g_{N}$ and $g_{\infty}$.

Finally, I stress that this mathematical example has two other features that make it a good prototype for my "singular limit" examples - i.e. my second and fourth, fractals and

\footnotetext{
${ }^{8}$ The convergence is pointwise not uniform: uniform limits of continuous functions are continuous.
} 
phase transitions; (hence my choice of it!). First: each example will have a two-valued quantity $f_{N}, N \in \mathbf{N} \cup\{\infty\}$, with $f_{N}=0$ for all $N \in \mathbf{N}$ and $f_{\infty}=1$. In fact, this quantity simply records the presence or absence of the emergent novel property; with presence encoded by $f=1$. So the jump in the value of $f$ corresponds to my claim (1:Deduce).

Second: in phase transitions (my fourth example, Section 7) there are physical quantities for finite models whose gradients grow without bound as $N \rightarrow \infty$, just like this example's gradients of $g_{N}$ in a neighbourhood of 0 . So the remarks here, about the unmysterious mathematical convenience and empirical accuracy of $g_{\infty}$, will apply-word for word!

Let me look ahead a little to Section 7, especially Section 7.1.2,B (if only to placate afficionados!). Consider the phase transition of a ferromagnet at sub-critical temperatures, as described by the Ising model with $N$ sites (in two or more spatial dimensions). The magnetization behaves, as a function of the applied magnetic field, very like this example's $g_{N}$. Thus suppose our variable $x$ represents the value of the applied field (in a given spatial direction). Then to a good approximation, $g_{N}$ represents the average magnetization (in appropriate units). So as the applied field passes from negative to positive values, the ferromagnet's magnetization flips from -1 (i.e. alignment with the field in the negative direction) to +1 (alignment in the positive direction). But for larger $N$, the ferromagnet "lingers longer": the larger number of sites gives it more "inertia" before the rising value of $x$ succeeds in flipping the magnetization from -1 to +1 . (Here, my qualifying phrase 'to a good approximation' refers to the Ising model's magnetization being a smooth function of the applied field (in fact given, in mean field theory, by the hyperbolic tangent function tanh), and so without sharp corners at $\pm 1 / N$ like my $g_{N}$.) Thus the magnetic susceptibility, defined as the derivative of magnetization with respect to magnetic field, is, in the neighbourhood of 0 , larger for larger $N$, and tends to infinity as $N \rightarrow \infty$ : compare the gradients of $g_{N}$ in my example. Very similar remarks apply to liquid-gas phase transition, i.e. boiling. Here the quantity which becomes infinite in the $N \rightarrow \infty$ limit, i.e. the analogue of the magnetic susceptibility, is the compressibility, defined as the derivative of the density with respect to the pressure 9

To sum up: I have dissolved the mystery about cases in which (i), i.e. the limit of the finite model, is not equal to (ii), the infinite model, and in which (ii) is empirically correct, by arguing that there are other quantities ( $g$ rather than $f$, in my notation) for which (i) is close to (ii) (and so, also, empirically correct). I can therefore turn to elaborating and endorsing the Straightforward Justification which I announced in Section 3.3.1; in short, mathematical convenience and empirical correctness. For I now maintain that it applies to all my four examples.

\footnotetext{
${ }^{9}$ Cf. also Kadanoff (2010, p. 20, Figure 5); Menon and Callender (2011) is a discussion of phase transitions concordant with mine, here and in Section 7 . You may well ask: Is my mathematical example also a good prototype for dissolving the corresponding alleged mystery in physics' other 'singular' limits, e.g. from optics, semiclassical mechanics and hydrodynamics? My view is: Yes. For a masterly philosopher's survey of the first two cases, cf. Belot (2005, Sections 3, 4 and Appendix).
} 


\subsubsection{Developing the Straightforward Justification}

This Justification consists of two obvious, very general, broadly instrumentalist, reasons for using a model that adopts the limit $N=\infty$ : mathematical convenience, and empirical adequacy (upto a required accuracy). So it also applies to many other models that are almost never cited in philosophical discussions of emergence and reduction. In particular, it applies to the many classical continuum models of fluids and solids, that are obtained by taking a limit of a classical atomistic model as the number of atoms $N$ tends to infinity (in an appropriate way, e.g. keeping mass density constant).

'Mathematical convenience and empirical correctness': merits that are so easy to state! But as all physicists know, and as echoed in the companion paper's discussion of good variables and approximation schemes: both can be very hard to attain -indeed, most of a physicist's work with a model is devoted to attaining them! But if they are attained by adopting the limit $N=\infty$, they surely justify using the limit. (At least, they do so, once we have disposed of any suspicious threat of mystery, such as refuting the atomic constitution of matter!)

Though the details vary widely among the countless models adopting some $N=\infty$ limit, this justification involves two themes that are common to so many such models that I should articulate them. The first theme is abstraction from finitary effects. That is: the mathematical convenience and empirical adequacy of many such models arises, at least in part, by abstracting from such effects. Consider (a) how transient effects die out as time tends to infinity; and (b) how edge/boundary effects are absent in an infinitely large system 10

The second theme is that the mathematics of infinity is often much more convenient than the mathematics of the large finite. The paradigm example is of course the convenience of the calculus: it is usually much easier to manipulate a differentiable real function than some function on a large discrete subset of $\mathbb{R}$ that approximates it 11 I shall just spell out two advantages which are endemic. We can begin with the simple case where we consider just the limit of the values, i.e. (i) of Section 3.2, so we set aside for the moment the infinite model, (ii) of Section 3.2.

Thus consider a model in which the actual value of the relevant quantity for realistic, i.e. large but finite, $N$, say $N=10^{23}$ - the value $v\left(f\left(10^{23}\right)\right)$ in Section 3.2] notation, taking the state as understood - is negligibly close to the $\operatorname{limit}_{\lim } \rightarrow \infty v(f(N))$. And let us assume that the value will remain close as $N$ grows: so the values obey $v\left(f\left(10^{23}\right)\right) \approx$

\footnotetext{
${ }^{10}$ As to (a), it is worth recalling the witty definition, attributed to Feynman, of that (invaluable but much-contested!) concept, 'equilibrium': 'the state the system gets into after the fast stuff [e.g. relaxation, transients] is finished and the slow stuff [e.g. Poincaré recurrence] has not yet started'. For apart from being witty, the mention of 'the slow stuff' echoes Section 2]s warning (4:Unreal). That is: we should beware that for very large times (not just for very large $N$ ) physical theories and models often become unrealistic. And as to both (a) and (b), recall also footnote 4s idea of intermediate asymptotics. Thus Feynman's witty definition should be revised along the lines 'the state the system gets into after both the really fast stuff, and the intermediate stuff, is finished and ...'.

${ }^{11}$ But smoothness is not everything! In some cases, as we saw with Section 3.3.2 $\mathrm{s} g_{\infty}$, a discontinuous function is more convenient than a continuous one.
} 
$v\left(f\left(10^{46}\right)\right) \approx v\left(f\left(10^{69}\right)\right)$ etc. Working with the limit rather than the actual value promises two advantages. (Here of course we set aside Section[2] warning (4:Unreal), that for many models, the values for vastly larger $N$ will eventually be unrealistic.)

The first is that it may be much easier to know, or at least estimate, the limit's value than the actual value - not least because of the first theme, the abstraction from finitary effects. And ex hypothesi, working with it involves a negligible inaccuracy about the actual value.

The second advantage is more theoretical, and will lead back to Section 3.2 s (ii), i.e. the value of a limit quantity on an infinite system. The idea here is that for most models and quantities $f$, there is, for a fixed $N$, not a single value $v(f(N))$, but a range of values, to be considered. That is: $v(f(N))$ is a function of some other variable which has so far been suppressed in my notation. And to make this function easily manipulated, e.g. continuous or differentiable so that it can be treated with the calculus, we often need to have each value of the function be defined as a limit (namely, of values of another function).

Continuum models of solids and fluids provide paradigm examples of this. For example, consider the mass density varying along a rod, or within a fluid. For an atomistic model of the rod or fluid, that postulates $N$ atoms per unit volume, the average massdensity might be written as a function of both position $\mathbf{x}$ within the rod or fluid, and the side-length $L$ of the volume $L^{3}$ centred on $\mathbf{x}$, over which the mass-density is computed: $f(N, \mathbf{x}, L)$. Now the point is that for fixed $N$, this function is liable to be intractably sensitive to $\mathbf{x}$ and $L$. In particular, if atoms are or contain point-particles the function will jump when $L$ is varied so as to include or exclude one such particle. That is: it will not be continuous in $\mathbf{x}$ and $L$. But by taking a continuum limit $N \rightarrow \infty$, with $L \rightarrow 0$ (and atomic masses going to zero appropriately, so that quantities like density do not "blow up"), we can define a continuous, maybe even differentiable, mass-density function $\rho(x)$ as a function of position - and then enjoy all the convenience of the calculus.

So much by way of showing in general terms how the use of an infinite limit $N=\infty$ can be justified-but not mysterious! At this point, the general philosophical argument of this paper is complete! The subsequent Sections present my examples. It will be clear that each example represents a large field of study. So to save space, I will have to be brutally brief, both about the examples' details and about references.

\section{The method of arbitrary functions}

My first example is the method of arbitrary functions in probability theory. It is a venerable tradition, initiated by Poincaré in his Calcul de Probabilities (1896), and developed by many authors including Borel, Fréchet and Hopf. Recent presentations include Engel (1992) and Kritzer (2003); and von Plato (1983, 1994, pp. 168-178) summarizes the history. But until recently it seems to have been largely neglected in the philosophy of probability, despite its offering an attractive way to reconcile non-trivial probabilities (i.e. probabilities that are neither 0 nor 1) with determinism at an 'underlying' level - and 
despite being the topic of Reichenbach's dissertation.12

The main idea of the method is best introduced by an example, and I will follow Poincaré (and most discussions) in choosing a roulette wheel, with alternating arcs of red and black (Section 4.1). Thus we will be concerned with the probability that the wheel stops with a red (respectively, black) arc opposite a pointer. For this example, the main idea will be that under certain assumptions, this probability tends to 0.5 , as the number $N$ of arcs goes to infinity - whatever the details of the spinning and slowing of the wheel. Section 4.1 will also discuss how this result can be generalized. Then in Section 4.2, I describe how this equiprobability in the limit $N \rightarrow \infty$ counts as emergent behaviour in my sense, and how it illustrates my claims, (1:Deduce) etc.

\subsection{Poincaré's legacy}

\subsubsection{Poincaré's roulette wheel}

Suppose that a roulette wheel with arcs of red and black is spun many times, eventually coming to a stop with a red or a black arc opposite a pointer. We suppose that it is spun using various unknown initial conditions, i.e. initial positions relative to the pointer and initial angular velocities; and that it is slowed and eventually stopped by some unknown regime of friction. If this is all we know, we can conclude essentially nothing about the long-run frequency (or probability, in any sense) of it stopping at Red (i.e. with a red arc opposite the pointer). For the variety of initial conditions and the regime of friction, taken together, amount to an unknown profile of biassing. This profile might be expressed as a function giving, for each arc, the probability of the wheel stopping there. And for all we have so far assumed, this function might make Red very probable (frequent) - or very improbable (infrequent).

But suppose we also assume that:

(i): there are very many alternating arcs of red and black;

(ii): whatever the unknown profile of biassing might be, it favours and disfavours large segments, i.e. segments each of which contains many red and many black arcs;

(iii): within one of these large segments, the bias is not too "wiggly" in the sense that two adjacent arcs get nearly equal biasses.

Then we can be confident that the long-run frequency of Red (and of Black) is about $50 \%$. For assumptions (i) to (iii) mean that if the profile is expressed as a probability function, each of its peaks (corresponding to a favoured segment) contains many red and many black arcs - and so do each of its troughs (corresponding to a disfavoured segment). Thus the contribution of any peak to the overall probability (or frequency) of stopping at Red will be about equal to the peak's contribution to the probability of stopping at Black; and similarly for any trough. So summing over all the peaks and troughs, the honours

\footnotetext{
${ }^{12}$ I say 'until recently' for two reasons. First: Strevens (2003) has revived the main idea; though he is wary of the philosophical value of theorems about limiting behaviour, which figure prominently in the tradition and which I will emphasize. For assessments of Strevens, cf. Colyvan (2005) and Werndl (2010). Second: some recent papers revive the main idea: Sober (2010), Frigg and Hoefer (2010), Myrvold (2011).
} 
will be about even between Red and Black: there will be approximate equiprobability. To sum up: (i) to (iii) imply that the idiosyncrasies of the biassing profile get washed out.

This is a beautiful and compelling idea; (originally due, apparently, to an 1886 book by von Kries; cf. von Plato 1983, p. 38; 1994, p. 169). Expressing it in general and probabilistic terms, we expect the following. Let a sample space $(X, \mu)$ be partitioned into two subsets, say $R$ and $B$, in a very "intricate" or "filamentous" way. Then for any probability density function $f$ that is not too "wiggly" (say: whose derivative is bounded: $\left.\left|f^{\prime}\right|<M\right)$ the probabilities of $R$ and $B$ are about equal:

$$
\int_{R} f d \mu \approx \int_{B} f d \mu \approx \frac{1}{2}
$$

And we expect: that, for any bound $M$ on the derivative of the density $f$, as the partition becomes more intricate or filamentous, the difference from exact equiprobability (and so to both probabilities equalling $\frac{1}{2}$ ) will tend to 0 .

Indeed, Poincaré (1912, p. 148ff.) turned this idea into a theorem, for a simple model of the roulette wheel. So we take $X$ to be the circle $[0,2 \pi]$, and the intricate partitioning of $X$ to be the division into $N$ equal intervals, labelled alternatingly 'red' and 'black'. We assume the distribution of the point $x \in X$ at which the wheel stops (i.e. which is eventually opposite the pointer) is given by a probability density function $f:[0,2 \pi] \rightarrow \mathbb{R}$. We assume that $f$ is differentiable, and its derivative is bounded by $M$, i.e. $\left|f^{\prime}\right|<M \in \mathbb{R}$. This of course makes precise assumptions (ii) and (iii) above 13 Then Poincaré showed:

For any $M \in \mathbb{R}$, for all density functions $f$ with derivative bounded by $M$, $\left|f^{\prime}\right|<M$ : as $N=$ the number of arcs goes to infinity:

$\int_{R} f d \mu \equiv \operatorname{prob}($ Red $) \rightarrow \frac{1}{2} ;$ and $\int_{B} f d \mu \equiv \operatorname{prob}($ Black $) \rightarrow \frac{1}{2}$.

To sum up: any biassing profile, no matter how wiggly, i.e. sensitive to the wheel's angular position (no matter how large $M$ ), can be washed out, so as to give equiprobability up to an arbitrary accuracy, by a sufficiently intricate partition, i.e. by a sufficiently large $N$.

\subsubsection{Generalizations: statistical stability}

Subsequently, Poincaré's theorem was generalized in two main ways. The first way was historically earlier and is less connected to later developments, especially of probabilistic methods in the study of dynamical systems. But it is easier to report since its conception of the parameter $N$ is very close to Poincaré's original: it measures the fineness of the partition of the sample space. In the second way, on the other hand, one takes a different limit, usually depending on the details of the dynamical system concerned.

I will now sketch both ways. But as regards illustrating my claims about emergence, I should stress the following points.

\footnotetext{
${ }^{13}$ We might also assume that the support of $f$ intersects all $N$ cells of the partition. This is one way (among several) to represent the natural requirement that the wheel is spun fast enough, at least sometimes, to prevent it stopping after just a few arcs have passed the pointer.
} 
(a) The illustrations do not need any of these generalizations; so the reader uniniterested in probability theory can now skip to Section 4.2.

(b) The first way leads to illustrations of my claims that are exactly parallel to the original illustration given by Poincaré's theorem: a happy circumstance, since it supports my view that my claims have a wide validity.

(c) The second way also illustrates my claims. But because a different, and even system-dependent, limit is taken, these illustrations are rather different from the Poincaré original. So to save space, I will not pursue the details.

(d) Poincaré's theorem and its generalizations (in both ways) are very suggestive for the philosophy of probability. As we will see, they hint that even with an underlying determinism, taking an appropriate limit can define non-trivial probabilities that are "objectively correct". But again, to save space, I must make a self-denying ordinance about this.

The first way generalized the assumptions of the model of the wheel, and adapted them to other chance set-ups. At first the conditions on the initial density function $f$ were weakened, by authors such as Borel and Fréchet. In short, Borel assumed merely that $f$ was continuous; and Fréchet merely that it was Riemann-integrable.

As to other chance set-ups, one paradigm example, which had the merit of extending the method of arbitrary functions to densities of more than one variable, was Buffon's needle. In this problem a person throws a needle of length $l$ on to a table on which a pattern of parallel lines at a distance $d(d>l)$ has been ruled. One asks: what is the probability that the needle lands so as to intersect one of the lines? The elementary treatment assumes that the point where the centre of the needle lands has a uniform probability density (in the interval $[0, d]$ for simplicity); and similarly that the angle between the needle and the lines is uniformly distributed. It then follows by an elementary argument that the probability of intersection is $2 l / d \pi$.

But it is more realistic to assume that there is some unknown ("arbitrary") density function, perhaps peaked near the centre of the table, for the point where the centre of the needle lands 14 Can we again apply von Kries' and Poincaré's idea that a more and more intricate partition of the sample space (here, the table) will wash out the influence of the peaks (and troughs) of the unknown density function? Yes! Borel indicated, and Hostinský showed in detail, that one can recover the familiar answer, $2 l / d \pi$, by taking the limit as the number $N$ of lines on the table goes to infinity. For this theorem, Hostinský assumed that the partial derivatives of the density function exist, are continuous and are bounded. And he takes the limit, $N \rightarrow \infty$, while (i) the table size is constant, so that the lines' separation $d$ goes to zero, and (ii) the ratio $l / d$ is constant.

At this point, we must concede that the theorems reported so far have an obvious limitation: the limit, $N \rightarrow \infty$, is unrealistic. The number of arcs on a roulette wheel, and the number of parallel lines on any table, is in fact fixed. (So this sense of being unrealistic is more straightforward, and in practice arises for much smaller $N$, than the idea of running up against the atomic constitution of matter, involved in my (4:Unreal) of

\footnotetext{
${ }^{14}$ Similarly, one might say, for the angle at which the needle lands. But I will not pursue how to relax this assumption.
} 
Section 2.) Can we respect this fact, and yet still apply our initial idea that an intricate partition of the sample space washes out the influence of the peaks and troughs of an unknown density function?

As I see matters, there are two broad strategies one can adopt. Both are important; and fortunately, they are compatible. The first strategy is piecemeal, and takes no limits. One models each chance set-up as realistically as one wishes or is able to; and then calculates, perhaps numerically, how wiggly (in some sense) the density function could be, while yielding approximately the probabilities we observe and-or desire - e.g. for the roulette wheel, equiprobability of Red and Black. This strategy is obviously sensible; and in Section 4.2.2 we will see how it illustrates my claim (2:Before). But for now, I turn to the other strategy.

This is what I called the 'second way' of generalizing Poincaré's theorem. In short: to derive the observed or desired probabilities, a different limit is taken. This strategy can also be piecemeal: the details of the chance set-up suggest what limit to take. I shall briefly report two impressively neat examples of this: Hopf's analysis of the roulette wheel, and Keller's analysis of coin-tossing. Then I shall report how this second way leads to the important idea of statistical stability.

Hopf's idea is that for a roulette wheel with a fixed number $N$ of arcs, the equiprobability of Red and Black will follow from allowing higher and higher initial angular velocities. Thus the basic insight is that even with $N$ fixed, a higher initial angular velocity implies that the width of an interval of velocities that lead to a specific arc stopping opposite the pointer is smaller. Or to make the same point at the opposite extreme: with just a few arcs (say, two!), and initial angular velocities so small that at most one rotation occurs, even a ham-fisted croupier can fix the wheel, i.e. guarantee stopping at Red, or at Black. In more detail: Hopf considers the total angle $\theta \in[0, \infty]$ through which some fiducial point on the wheel's circumference turns before the wheel stops. Higher initial angular velocities will make $\theta$ larger; and Red or Black is determined by $\theta \bmod 2 \pi$. The regime of spinning and friction is summarized in an unknown density function $f$ on the initial angular velocity $\omega$, with bounded support. But higher velocities are considered by translating $f$ by a constant $C$, i.e. by defining $f^{*}(\omega):=f(\omega-C)$; and by letting $C \rightarrow \infty$. Hopf also allows the frictional force (the braking) to depend, not only on the present angular velocity, but also on the angle so far turned through; that is, he allows for an unbalanced wheel. Hopf then proves that as $C \rightarrow \infty$, the distribution of $\theta$ mod $2 \pi$ tends to being uniform on $[0,2 \pi]$.

Keller gives a broadly similar analysis of coin-tossing (1986; developed by Diaconis et al. 2007). He takes the coin to be a circular lamina which is initially horizontal: it is tossed in a vertical line with an initial angular velocity $\omega$ and initial vertical velocity $u$, and falls under gravity onto a horizontal table where it settles with either Heads or Tails facing upward. Like Poincarés or Hopf's wheel, the sample space of initial conditions is intricately partitioned into subsets that lead eventually, and deterministically, to Heads or to Tails. But like Buffon's needle, the sample space is two-dimensional. It is the positive quadrant of the $(\omega, u)$-plane. So the probabilities of Heads and Tails are given by integrating over the Heads and Tails subsets, respectively, an unknown density function 
$f(\omega, u)$, which Keller takes to be continuous.

Keller shows that the pattern of Heads and Tails subsets is like a "hyperbolic zebra". Each subset is a thin strip lying along one of a series of hyperbolas, i.e. curves like $\omega=n K / u$ with $n$ a natural number. Besides, Heads strips alternate with Tails strips; and for higher values of $\omega$ and $u$ (i.e. as we move North-East in the positive quadrant), the strips become thinner. This means that, in the now-familiar way, the integral of $f$, for Heads or for Tails, over these higher values becomes less sensitive to wiggles in $f$. That is: as the support of $f$ (or even just $f$ 's "preponderant weight") tends "North-East", Heads and Tails tend towards being equiprobable - whatever the density function.

Agreed, you might object that these analyses of Hopf and Keller, though neat, are again unrealistic. No roulette wheel is spun, and no coin is tossed, arbitrarily fast! But the reply is clear. It has two parts. Analyses like Hopf's and Keller's can give information about the speed of convergence towards their limit; and this can reassure us that realistic initial conditions lead to the desired probabilities (here: equiprobability), up to a good accuracy, for a wide class of density functions. Here of course we return to two previous themes:

(i) in general terms, the two merits of Section 3.3.3 s Straightforward Justification of taking a limit: mathematical convenience and empirical success; and

(ii) specifically, the value of modelling without taking a limit, i.e. the first strategy above, and my claim (2:Before). Recall my remark above that the two strategies are compatible.

Finally, Hopf's and Keller's analyses prompt the idea of statistical stability, which has been very important for the probabilistic study of dynamical systems. I will not go in to the measure-theoretic technicalities (about absolute continuity and types of convergence) that are needed for an exact definition, but just convey the main idea. (This occurs, under the label 'statistical regularity' in Hopf's own analysis of the roulette wheel.) The general scenario is that we are given: (i) two probability spaces $(X, \mu)$ and $(Y, \nu)$, i.e. $\mu, \nu$ are probability measures on appropriate fields of subsets of $X, Y$ respectively; (ii) a family of maps $F_{\lambda}: X \rightarrow Y$, labelled by a parameter $\lambda \in \mathbb{R}$ or perhaps $\in \mathbf{N}$. Thus in our examples above, $X$ was the space of initial conditions and $Y$ was the two element space $\{$ Red, Black $\}$ or $\{$ Heads, Tails $\}$; and each $F_{\lambda}$ is a deterministic map sending an initial condition $x \in X$ to an outcome $y \in Y$.

Returning to the general scenario: $\mu_{\lambda}:=\mu \circ F_{\lambda}^{-1}$ is a probability measure on $Y$, and we can ask whether there is a measure on $Y$ to which $\mu_{\lambda}$ converges as $\lambda \rightarrow \infty$ : or even a measure on $Y$ to which $\mu_{\lambda}$ converges, for all $\mu$ on $X$ in some suitable class. If so, we say the family $F_{\lambda}$ is statistically stable. In studying complicated, even "chaotic", deterministic systems, this idea has an important special case: namely, $X=Y, \mu=\nu, \lambda \in \mathbf{N}$ and the family $F_{\lambda}$ arises just by iterating a map $T: X \rightarrow X$, i.e. $F_{\lambda}:=T^{\lambda}$ represents a discrete-time evolution. In this case, the limit measure, $\mu^{*}$ say, characterizes the longtime statistical behaviour of the system. In particular, it is readily shown to be invariant under the time-evolution. That is, $T$ induces an evolution $P_{T}$ on measures (and their densities) in the natural way, and we have: $P_{T}\left(\mu^{*}\right)=\mu^{*}$. 


\subsection{The claims illustrated by emergent equiprobability}

I turn to describing how the limiting probabilities of Section 4.1 count as emergent behaviour in my sense, and how they illustrate my claims (1:Deduce), (2:Before) and (3:Herring) (listed in Section 1.2). As I announced, I will for simplicity emphasize the original Poincaré theorem, stated at the end of Section 4.1.1. But it will be clear how the claims are also illustrated by the generalizations given in Section 4.1.2, including the closing idea of an invariant limit measure $\mu^{*}$.

The illustrations unfold immediately, once we stipulate that the limiting probabilities are to be the emergent behaviour. For me, this means behaviour that is novel or surprising, and robust, relative to a comparison class. As discussed in the companion paper, this class is liable to be fixed contextually, and even to be vague or subjective - but nevermind, since there does not need to be an exact meaning of 'emergence'. Here I concede that the limiting probabilities, especially the equiprobability of Red and Black, or Heads and Tails, are not novel or surprising - though I submit that it is surprising that one can deduce them from an arbitrary density function. In any case, they are robust in a vivid sense: the whole point of the method of arbitrary functions is that they are invariant under a choice of a density function from a wide class.

\subsubsection{Emergence in the limit: with reduction - and without}

As to (1:Deduce): we have 'reduction as deduction' in as strong a sense as you could demand-provided we take the limit. Thus for Poincaré's theorem, we take $T_{t}$ to be just the statement of equiprobability in the limit of infinite $N$, and $T_{b}$ to be a model of the wheel, including enough measure theory and calculus to cover both: (i) the postulation of various possible density functions $f$ on $[0,2 \pi]$; and (ii) consideration of the infinite limit $N \rightarrow \infty$. And similarly for Section 4.1 s other examples.

(1:Deduce) also concerns "the other side of the coin": how the emergent behaviour, here equiprobability, is not deducible, if we do not take the limit but instead confine $T_{b}$ to finite $N$. This also is illustrated by Section 4.1. Thus in particular, for Poincaré's roulette wheel: For any finite $N$, no matter how large, equiprobability will fail, as badly as you may care to require, for a sufficiently "wiggly" density function, i.e. a sufficiently position-sensitive biassing regime. That is, we have:

For all $\varepsilon>0$, for all positive integers $N$, there is $M \in \mathbb{R}$ and a density function $f$ with $\left|f^{\prime}\right|<M$ such that: $\int_{R} f d \mu \equiv \operatorname{prob}($ Red $)>1-\varepsilon$.

So here is emergence without reduction to a weaker finitary $T_{b}$. Since this weaker $T_{b}$ is a salient theory, one can be tempted to speak of irreducibility. Similarly for Section 4.1 $\mathrm{s}$ other examples.

It is worth displaying the two sides of (1:Deduce)'s "coin" - equiprobability's deducibil-

ity in the limit, and its non-deducibility before - in terms of a shift of quantifiers. Thus the "form" of Poincaré's theorem is: 


$$
\begin{aligned}
& \forall \varepsilon>0, \forall M \in \mathbb{R}, \forall f \text { with }\left|f^{\prime}\right|<M, \exists N \text { s.t. } \forall N^{*}>N:\left|\int_{R ; N^{*} \operatorname{arcs}} f d \mu-\frac{1}{2}\right| \\
& <\varepsilon ;
\end{aligned}
$$

while "the other side of the coin" is:

$$
\forall \varepsilon>0, \forall N, \exists M \in \mathbb{R} \text {, and } f \text { with }\left|f^{\prime}\right|<M, \text { s.t.: }\left|\int_{R ; N \operatorname{arcs}} f d \mu-\frac{1}{2}\right|>\varepsilon \text {. }
$$

One can easily check that in Section 4.1]s other examples, including Buffon's needle, Hopf's roulette wheel and Keller's tossed coin, the two sides of (1:Deduce)'s "coin" involve a similar quantifier-shift.

Finally, I stress the point announced in Sections 1.1 and 3.1 that the limits we are concerned with are in no way singular - so a singular limit is not necessary for emergence. Nor is there any infinite system corresponding to $N=\infty$ (i.e. $\sigma(\infty)$ in Section 3.1 s notation). For the roulette wheel, that would mean a division of $[0,2 \pi]$ in to a denumerable number of equal-length segments! And similarly for the other limits: e.g. Hopf's roulette wheel spun, or Keller's coin tossed, with an infinite initial angular velocity 15

\subsubsection{Emergence before the limit}

(2:Before) claims that before the limit, there is emergence in a weaker but still vivid sense. Here the weaker sense is approximate rather than exact equiprobability, for some realistic model of the roulette wheel (or other chance set-up). So we already saw in Section 4.1.2 how the method of arbitrary functions illustrates this claim: namely, in the discussion of the finite parameter case, both (i) as a first strategy for defending Poincaré's roulette wheel and (ii) as a reply to the parallel objection to Hopf or Keller, that no wheel is spun, no coin is tossed, arbitrarily fast. For both (i) and (ii), we calculate, perhaps numerically, how wiggly (in some sense) the density function could be, while yielding approximately the probabilities we observe and-or desire - e.g. for the roulette wheel, equiprobability of Red and Black.

Speaking of desire raises issues of engineering: indeed, of the profitability of casinos. We know that casinos manage to get profitably close to equiprobability, with some small number, $N \approx 50$, of arcs. And we surmise that even if they had a worryingly wiggly $f$, they could get profitably close to equiprobability by putting $N$ up to say about 200; or-following Hopf's idea - by spinning the wheel, on average, some two to three times faster. Here we meet the multi-faceted, even interest-relative, even subjective, question: how close is close enough? 'Close enough for all practical purposes': but what exactly are the practical purposes? How wiggly an $f$ need the casino guard against?

But I submit that this is a question for casino-owners - who can no doubt pay staff well enough to answer it accurately for them. At our (typically philosophical!) level of

\footnotetext{
${ }^{15}$ I said there can be no division of $[0,2 \pi]$ in to a denumerable number of equal-length segments. No sooner said than doubted - as so often in philosophy. I am grateful to Alan Hajek for pointing me to Edward Nelson's adaptation of the ideas of non-standard analysis to probability theory; cf. Nelson (1987, especially Chapters 4 to 7 ).
} 
generality, we do not need to try and answer it. For us, it is enough that given a resolution of this and similar questions, including vaguenesses, we get a notion of approximate equiprobability, which can indeed be deduced from a $T_{b}$ with parameters that are not only finite, but also realistic. In particular, $T_{b}$ can imply profitable - for the gamblers: indiscernible - closeness to equiprobability, using some $N \approx 50$ arcs on the roulette wheel, and an initial velocity of some $10 \pi$ to $30 \pi$ radians per second ( 5 to 15 revolutions per second).

\subsubsection{Supervenience is a red herring}

I turn to my third, ancillary, claim (3:Herring). Namely: although various supervenience theses are true, they yield little or no insight into emergence, or more generally, into "what is going on" in the example.

This is well illustrated by Poincaré's roulette wheel, and Section 4.1 s other examples. For any sequence of spins of the wheel, with any number $N$ of $\operatorname{arcs}$, and any regime governing its initial velocities, the frequency of Red is of course determined by, supervenient upon, all the microscopic details of the wheel and its many spinnings 16 This supervenience thesis holds for a finite sequence of spins; or an infinite one, with frequency defined as limiting relative frequency. And there are analogous supervenience theses for probability, rather than frequency: the probability of Red is determined by the details of the wheel, especially the choice of probability density function. Similarly of course, for coin-tosses, and the frequency or probability of Heads.

I submit that these supervenience theses, whether for frequency or probability, shed no light on the matters at hand. For they make no connection with the basic idea of the method of arbitrary functions: that intricate partitions of a sample space can wash out the peaks and troughs of an unknown density function, and secure robust probabilities. This is a good illustration of my general reasons (in Section 1.2) for supervenience theses' irrelevance: that they make no connection between their idea of a variety, perhaps even infinity, of ways to have the higher-level property $P$, and the limit processes on which the example turns. Thus here, $P$ is the property that a frequency or probability of Red (or of Heads) is $\frac{1}{2}$, or is the property of two events being equiprobable; and the example's limit processes are the number of arcs, or the initial velocities, going to infinity, so as to implement the basic idea of washing out peaks and troughs. Or we can eschew the limit and use only finite parameters, as in (2:Before). But again, these supervenience theses shed no helpful light 17

\footnotetext{
${ }^{16}$ In Section 4.1, we assumed, for the most part implicitly, that these details were based on classical mechanics. But the same supervenience thesis would hold if we assumed instead that they were based on quantum theory. At least, this is so if we set aside the quantum measurement problem, which threatens to deny us any definite macroscopic events. The companion paper discusses some dangers in the idea of supervenience on the microscopic details, "whatever they might be".

${ }^{17}$ A caveat. I agree that these supervenience theses are relevant to the philosophy of probability, especially for an empiricist. For example: if we maintain that the empiricist should accept the model's microscopic details, say because they are "occurrent", then the supervenience theses for frequencies support the idea that they should also accept frequencies - as a metaphysical free lunch, as people say. But in (d) at the start of Section 4.1.2 I foreswore the philosophy of probability: for some discussion in
} 


\section{$5 \quad$ Fractals}

My second example is fractals, or rather, one small aspect of this large field: namely, the idea that a set of spatial points, i.e a subset of $\mathbb{R}^{n}(n=1,2, \ldots)$, can have a dimension that is not an integer. As we shall see, one can define various notions of dimension; and much of the discussion and results carry over to spaces more general than Euclidean space $\mathbb{R}^{n}$. However, I will emphasise one notion of dimension, scaling dimension (also known as: similarity dimension), and confine myself to $\mathbb{R}^{n}$. Even a very short introduction to this topic (Section 5.1) will be enough to illustrate my claims. For my first three claims, details are in Section 5.2. The discussion is similar to that in Section 4.2 18

But as I mentioned in Section 2, I propose for fractals to also discuss my fourth claim (4:Unreal): that for large but finite $N$, the example becomes unrealistic - for reasons that are usually ignored in discussions of emergence. I do this in Section 5.3. This will mean that in Sections 5.1 and 5.2, the pure mathematics of dimension in Euclidean geometry will be prominent: the empirical world will come to the foreground only in Section 5.3. For in this fractals example, large $N$ corresponds to very small length-scales; so that here, (4:Unreal) amounts to a 'No' answer to the question 'Is fractal geometry the geometry of nature?' In other words: (4:Unreal) denies that fractal descriptions of physical objects are literally true: a denial which my first three claims can largely ignore. Section 5.4 will sum up.

\subsection{Self-similarity and dimension as an exponent}

The key innovation of fractals is to extend, from familiar geometric objects such as squares and cubes to much more "irregular" sets, two related ideas: (i) self-similarity and (ii) dimension as an exponent.

Recall that a square with edge $l$ is the union of $l^{2}$ unit squares; e.g. a square whose edge is $l=3$ units long is the union of $3^{2}=9$ unit squares. And a cube with edge $l$ is the union of $l^{3}$ unit cubes; e.g. a cube whose edge is $l=3$ units long is the union of $3^{3}=27$ unit cubes. These examples exhibit both the ideas (i) and (ii), as follows.

(i): The square or cube is a union of smaller copies of itself; and the decomposition involved can be iterated indefinitely -imagine repeatedly shrinking the unit of length $l$ by some factor.

(ii): In the formula for the measure (area, volume) of the object (i.e. the number of unit building blocks in it), the dimension occurs as an exponent, and takes the same value, however fine the decomposition i.e. however small we choose the unit of length.

$$
\text { number of unit blocks in object with edge } l=l^{\text {dimension of object }} \text {. }
$$

the context of the method of arbitrary functions, cf. the papers by Frigg and Hoefer, and Myrvold cited in footnote 12 .

${ }^{18} \mathrm{I}$ should mention a reason for restricting attention to Euclidean space $\mathbb{R}^{n}$. Namely: Euclidean geometry admits similarity (of triangles and other figures), while non-Euclidean geometries in general do not; and on our approach, the definition of fractals needs the idea of similar figures. Section 5.3 will return to this point. 
So the main idea of fractals is that similarly:-

$\left(i^{\prime}\right)$ : Some "irregular" sets of points are unions of smaller copies of themselves; where, again, the decomposition involved can be iterated indefinitely. Among these sets will be some famous examples, which were treated as "pathological" when first explored some hundred years ago: in particular, the Cantor 'middle thirds' set $C$ which is a subset of the unit interval $[0,1] \subset \mathbb{R}(1872)$, and the Koch snowflake $K$ which is a subset of the unit square (1906).

(ii'): Applying the idea of eq. 5.1 to such sets, we find that they have non-integral dimensions. For example, the Cantor set has dimension about 0.63, and the Koch snowflake has dimension about 1.26 .

These ideas are connected to my themes of emergence and reduction, owing to the fact that these sets are defined by taking a limit $N \rightarrow \infty$ of an iterated process of definition. Thus in Section 5.2 I will take non-integral dimension to be the emergent (i.e. novel and robust behaviour), which is deduced (and so reduced!) in the limit.

I shall now develop ideas (i) and (ii), especially eq. 5.1, more formally. But how fractals illustrate my claims about emergence and reduction does not depend on these details, and the reader uninterested in geometry can now skip to Section 5.2. But I should also stress, on the other hand, that what follows is the merest glimpse of the modern theory of dimension. I shall rein in the exposition, and say only enough: (a) to define the scaling dimension, and see how it can be non-integral (Section 5.1.1), and (b) to sketch how scaling dimension relates to other concepts of dimension (Section 5.1.2).

\subsubsection{Examples: scaling dimension}

I begin by defining the Cantor set and Koch snowflake. This will show that they are self-similar, i.e. unions of smaller copies of themselves; and this will imply that using eq. 5.1 s idea of dimension as exponent, both these sets have a non-integer dimension. Then I give a general definition of scaling dimension.

5.1.1.A: The Cantor set $C$ :- This is defined as the intersection of infinitely many other subsets, which we will call 'stages', labelled $0,1,2, \ldots$ The unit interval $[0,1]$ is stage 0 . After stage 0 , each later stage is obtained by deleting the open middle third of each closed interval of its predecessor. So stage 1 is $[0,1]$, minus its open middle third. That is: stage 1 is $\left[0, \frac{1}{3}\right] \cup\left[\frac{2}{3}, 1\right]$. Then stage 2 is defined by deleting the open middle third of each of $\left[0, \frac{1}{3}\right]$ and $\left[\frac{2}{3}, 1\right]$. So stage 2 consists of four disjoint closed intervals: it is the set $\left[0, \frac{1}{9}\right] \cup\left[\frac{2}{9}, \frac{1}{3}\right] \cup\left[\frac{2}{3}, \frac{7}{9}\right] \cup\left[\frac{8}{9}, 1\right]$. And so on. Thus stage $N$ is the union of $2^{N}$ intervals, each interval being of length $\left(\frac{1}{3}\right)^{N}$. So the total length of stage $N$ is $2^{N} \times\left(\frac{1}{3}\right)^{N} \equiv\left(\frac{2}{3}\right)^{N}$. So as $N$ goes to infinity, the length of stage $N$ goes to 0 .

$C$ is defined to be the intersection of all the stages. Thus $C$ contains those real numbers between 0 and and 1 whose ternary expansion (i.e. using digits $0,1,2$ ) has no digit 1 : so $C$ is uncountable. Agreed, $C$ is hard to visualize! Its topological properties include: it is closed, it is nowhere-dense (i.e. its closure has an empty interior) and its complement is a dense subset of $[0,1]$. 
Now we apply to $C$ the idea of eq. 5.1. Think of $C$ as the unit block of "Cantor type". And observe that $C$ is the union of two shrunken copies of itself, each smaller by a factor of 3. That is: one shrunken copy is built by applying the infinite 'delete and take intersection' process to $\left[0, \frac{1}{3}\right]$, and the other shrunken copy by applying the process to $\left[\frac{2}{3}, 1\right] 19$

This observation can be reproduced at the next scale up. That is: we can define the "Cantor type" object of scale 3 , call it $C^{\prime}$, as the set that results from applying the infinite 'delete and take intersection' process to $[0,3]$, rather than to $[0,1]$. Then just as our original $C$ is the union of two shrunken copies of itself, each smaller by a factor of 3 , so also is $C^{\prime}$. That is: $C^{\prime}$ is the union of two unit-size Cantor sets. Now we apply the idea of eq. 5.1, getting

number of unit Cantor sets in Cantor object of scale $3 \equiv 2=3^{\text {dimension of } C}$.

Now we recall that for any $\operatorname{logarithm}$ base $a, b=c^{\left(\log _{a} b / \log _{a} c\right)}$, so that in the case of interest: $2=3^{(\log 2 / \log 3)}$. Here we drop the suffix stating the base, since the ratio of logarithms is independent of the base. That is: the dimension of $C$ is $\log 2 / \log 3$ : which is about 0.63 .

5.1.1.B: The Koch snowflake $K$ :- This also has an iterative construction. Roughly speaking: we erect smaller and smaller equilateral triangles in the middles of the sides of a polygon, and define $K$ as the limit. Thus stage 0 is an equilateral triangle. Stage $N+1$ is constructed from stage $N$ by replacing each line segment of stage $N$ by 4 line segments, each one-third the length of the original. It follows that the perimeter of the polygon grows without bound: if $P$ is the perimeter of the initial triangle, then stage $N$ consists of $3 \times 4^{N}$ segments each of length $P / 3^{N+1}$, so that its perimeter is $(4 / 3)^{N} P$.

So this is different from the Cantor set in that $K$ is not itself the union of similar smaller snowflakes. But each "side" of $K$ is the union of four smaller similar curves, each smaller by a factor 3. So applying again the idea of eq. 5.1, we get:

$$
4=3^{\text {dimension of } K} \quad \text { so that: } \quad \text { dimension of } K=\frac{\log 4}{\log 3} \approx 1.26 .
$$

5.1.1.C: Scaling dimension defined:- With these examples as motivation, I proceed to a general definition. The main effort is in defining the preliminary notion of self-similarity. For in general we need to allow that the smaller copies (of which the object, i.e. set, we are concerned with is a union) overlap, i.e. have non-empty intersection. But we require them to overlap "minimally" in the sense that their intersection is of lower dimensionin the usual integer-valued sense! - than the copies themselves. Examples include: two continuous curves that have a finite set of points in common; two rectangles that have parts of the boundaries in common.

\footnotetext{
${ }^{19}$ This observation can be iterated "downward". $C$ is also the union of $2^{2}$ shrunken copies of itself, each smaller by a factor of $3^{2}$. And $C$ is the union of $2^{3}$ shrunken copies of itself, each smaller by a factor of $3^{3}$; and so on... for each $N, C$ is the union of $2^{N}$ shrunken copies of itself, each smaller by a factor of $3^{N}$.
} 
For the moment, I will take the usual integer-valued notion of dimension for granted; (Section 5.1 .2 will rehearse a standard definition of it). Then we say that a set $X \subset \mathbb{R}^{n}$ is a almost-disjoint union of two sets $Y, Z$ iff $X=Y \cup Z$ and $Y \cap Z$ has lower dimension than the dimensions of $Y$ and $Z$. One similarly defines almost-disjoint unions of more than two sets. And one defines $X$ to be self-similar if it is an almost-disjoint union of shrunken copies of itself. Here 'shrunken copies' can be made precise by using the vector space structure of $\mathbb{R}^{n}$ : (i) to scalar-multiply the vectors in the set $X$ by a common contraction factor, and (ii) to translate the resulting shrunken copies out of coincidence with one another, so as to give an almost-disjoint union. Thus we say: $X$ is self-similar if it is the almost-disjoint union of $m$ copies of $X$, each contracted by a common factor $k$, and then translated by a (non-common) vector $v$. Thus in an obvious notation

$$
X=\cup_{i=1}^{m}\left[\frac{1}{k} X+v_{i}\right]
$$

Then we define the scaling dimension of $X$ to be: $\log m / \log k$.

\subsubsection{Generalizations: three other concepts of dimension}

Our definition of scaling dimension, eq. 5.4, is limited to exactly self-similar objects. But the idea that a dimension occurring as an exponent in a power law can be non-integral can be developed for much more general kinds of object. These include: (i) allowing the contraction factor for the building-block set $X$ to be anisotropic (called 'self-affinity', instead of 'self-similarity'); and (ii) introducing probabilities governing the contractions and-or translations of $X$, so that one considers an ensemble of random fractals, almost all of which are not exactly self-similar.

These developments have both empirical and theoretical aspects: which have of course influenced one another over the years. In this Subsection, I round off our glimpse of the modern theory of dimension by sketching some of these developments: first the empirical, then the theoretical. There will be a common key idea: to substitute for Section 5.1.1]s contractions of a figure, the complementary idea of contracting a grid of lines (or planes or hyperplanes), or something analogous to such a grid, like a family of boxes or discs that appropriately cover the figure.

5.1.2.A: Empirical aspects:- Countless empirical studies have found power law behaviour with a dimension as a non-integral exponent. One famous example is Richardson's (1961) discussion of measuring the length of a coastline by traversing it from point to point, as if with a pair of dividers. Richardson envisages indefinitely improving the resolution, i.e. reducing the divider-distance. For a continuous curve, we would have the familiar limit: as the resolution length (divider-distance) $\delta \rightarrow 0$, the number of steps $n(\delta)$ needed to traverse the coastline grows unboundedly in such a way that the estimate of the length, $n(\delta) \delta$, tends to $l$ : where $l$ is the usual length of the curve, given by calculus as $l=\int d s$. We can express this as a power law with the curve's dimension $D=1$ as an exponent. Namely, we would have:

$$
n(\delta) \approx l / \delta \equiv l / \delta^{1} \equiv l / \delta^{D} \text { with } D=1 .
$$


But applying the dividers method to ever-larger scale maps suggests instead that as $\delta \rightarrow 0$, the estimated length $n(\delta) \delta$ increases without bound, i.e. $n(\delta) \approx$ constant $\times \delta^{D}$ with $D$ strictly greater than 1 . This is of course like Section 5.1.1 s discussion of the length, and the dimension, of (the side of) the Koch snowflake: except that intuitively a coastline has bays as well as promontories - concave portions as well as convex ones. But this can be modelled using random fractals, as mentioned in (ii) above.

There are many other examples of such empirical power laws: often, as in this example, with the quantity of interest, $f$ say, proportional to a power of a resolution $\delta$ : $f=$ constant $\times \delta^{D}$. In many cases, of course, the exponent represents, not length, area or volume, but some other physical quantity. But there are also plenty of cases where the exponent is a dimension (in our sense, not the more general sense of 'physical dimension'!). Thus Brady and Ball (1984) studied the dendritic growth of copper electrodeposited on to an initially pointlike cathode. They found that the volume (or mass) of copper was proportional to $R^{D}$, where $R$ is the radius and $D$ was about 2.43 - in good agreement with computer-simulations.

5.1.2.B: Theoretical aspects:- For my purposes, the main point here is that the modern theory of dimension recognizes several different concepts, and of course includes many theorems relating the agreements and differences in the dimensions assigned to various sets. I shall sketch three such concepts. As I mentioned at the start of this Subsection, they share a common general idea: viz. successively finer covers of the set in question, or something analogous like successively finer grids of lines or (hyper)planes.

I start, for the sake of completeness, with the traditional, i.e. topological, integervalued notion. My other two notions are the Hausdorff dimension and the box dimension. They are like scaling dimension, not just in taking non-integral values; but also in the general underlying reason for this, viz. some quantity showing power law behaviour. Besides, the dimension they assign to an exactly self-similar set, as in eq. 5.4, is equal to the scaling dimension: viz., in the notation of eq. 5.4, $\log m / \log k$ (Falconer 2003, pp. xxiv, 129; Hastings and Sugihara 1993, p. 31, 34, 40). So they are generalizations of scaling dimension, in the clear sense that if a set $X$ has a scaling dimension $D$, then it also has both Hausdorff and box dimension equal to $D$. But as I mentioned, each of these notions also applies to a much wider class of sets. Besides, they have in common that the power law behaviour occurs as a cover of a set, or something analogous like a grid of lines or planes, becomes finer. But they are inequivalent notions: for some sets, their values disagree.

Topological dimension: This can be defined for a general topological space; but I restrict myself to compact subsets of $\mathbb{R}^{n}$. There are various ways to motivate the definition. Among the clearest is to consider the task of covering the unit square with closed rectangles in such a way that as few rectangles as possible have points in common. Suppose we cover the square with a lattice of rectangles; (so the square is their almost-disjoint union). Then a point at a corner of the lattice is in four rectangles. If instead we stagger the rectangles, giving a brick-wall pattern, then each point at a corner is in only three rectangles. On the other hand, it seems this arrangement cannot be improved - except of course by making the rectangles so that we only need two, or even one(!), to cover the 
square. Similarly in three dimensions. A few minutes' reflection suggests that: (a) for covering the unit cube with arbitrarily small closed rectangular solids, one can arrange that no point in the cube is contained in more than four solids; but (b) for sufficiently small solids, at least four solids have a common point. Similarly, one naturally conjectures, for unit hypercubes $[0,1]^{n} \subset \mathbb{R}^{n}:$ (a) $[0,1]^{n}$ can be decomposed as an almost-disjoint union of arbitrarily small $n$-rectangles, in such a way that no more than $n+1$ of them have a common point; but (b) $n+1$ is the least such number, i.e. in any such decomposition of $[0,1]^{n}$, there must be a point common to at least $n+1$ of the $n$-rectangles.

This prompts the following definitions. Let $X \subset \mathbb{R}^{n}$. Let $\mathcal{U}$ be a cover of $X$ by finitely many sets, and let $\delta>0$. $\mathcal{U}$ is called an $\delta$-cover if each element of $\mathcal{U}$ has diameter less than $\delta$. (The diameter diam $U$ of a set $U$ is $\sup _{x, y \in U}|x-y|$.) The order, ord $\mathcal{U}$, of $\mathcal{U}$ is the natural number $m \in \mathbf{N}$ for which there is a point of $X$ belonging to $m$ elements of $\mathcal{U}$, but no point belonging to $m+1$ elements. Then we say that $X$ has topological dimension $m$ iff $m$ is the least integer for which, for any $\delta>0$, there is a finite closed $\delta$-cover of $X$ of order $m+1$.

This definition is the beginning of a rich theory. In particular, one shows that it gives the intuitive verdicts about familiar sets of points: finite sets of points, lines, planes and solids get dimensions $0,1,2$ and 3 respectively; and so on for $\mathbb{R}^{n}$. One shows that dimension thus defined is a topological invariant. It is also easy to check that the Cantor set has topological dimension 0 .

Hausdorff dimension: The definition proceeds in two main steps. (1): We first sum the diameters, raised to a power $s$, of the elements of a cover of the set $X$, and consider the limit as the supremum of these diameters goes to 0 . As the set $X$ varies, we get for fixed $s$ a function $H^{s}$, which is (an outer measure, and thereby) a measure on an appropriate field of sets - which includes the Borel sets. (2): These measures, parameterized by $s$, have the curious property that for any given set $X$, the value $H^{s}(X)$ is zero or infinity for most $s$. It is this property that yields the definition of the dimension. The details are as follows.

(1): Let $X \subset \mathbb{R}^{n}$ and $s>0$ and $\delta>0$. We define

$$
H_{\delta}^{s}(X)=\inf \Sigma_{i=1}^{\infty}\left(\operatorname{diam} U_{i}\right)^{s}
$$

where the infimum is over all countable $\delta$-covers $\left\{U_{i}\right\}$ of $X$. (One can check that $H_{\delta}^{s}$ is an outer measure on $\mathbb{R}^{n}$.) Now we let $\delta \rightarrow 0$ :

$$
H^{s}(X):=\lim _{\delta \rightarrow 0} H_{\delta}^{s}(X)=\sup _{\delta>0} H_{\delta}^{s}(X) .
$$

This limit exists; but may be infinite because $H_{\delta}^{s}$ increases as $\delta$ decreases. $H^{s}$ is an outer measure, and so restricts to a measure on the $\sigma$-field of $H^{s}$-measurable sets. This includes the Borel sets, and the measure is called Hausdorff s-dimensional measure.

(2): For any $X, H^{s}(X)$ is clearly non-increasing as $s$ increases from 0 to $\infty$. And if $s<t$, then $H_{\delta}^{s}(X)>\delta^{s-t} H_{\delta}^{t}(X)$. This implies that if $H^{t}(X)$ is positive, then $H^{s}(X)$ is infinite. So there is a unique value, $\operatorname{dim}_{H}(X)$, the Hausdorff dimension of $X$, such that

$$
H^{s}(X)=\infty \text { if } 0 \leq s \leq \operatorname{dim}_{H}(X) \text {; and } H^{s}(X)=0 \text { if } \operatorname{dim}_{H}(X) \leq s \leq \infty
$$


A rich theory ensues: for its beginning, cf. Falconer (2003, Chapter 2).

Box dimension: The idea is to count the minimum number $N(\delta)$ of closed $n$-cubes of a given edge-length $\delta$ that cover the set in question, and to consider the limit as $\delta \rightarrow 0$. In the now-familiar way suggested by the scaling dimension, $\log m / \log k$, of eq. 5.4, the dimension is defined as

$$
\lim _{\delta \rightarrow 0} \frac{\log N(\delta)}{-\log \delta}
$$

The minus sign is needed to make the dimension positive, since $\log \delta \rightarrow-\infty$ as $\delta \rightarrow 0$. In fact, we can work, equivalently and conveniently, with the smallest number of closed balls of radius $\delta$.

As to the conditions for the limit to exist, I here just recall that any sequence $\left\{a_{n}\right\}$ of real numbers has a lim inf and a lim sup (which may equal $\pm \infty$ ), defined as follows: lim inf $a_{n}$ is the number $a$ such that: (i) for all $\varepsilon>0, a_{n}$ is eventually forever greater than $a-\varepsilon$, i.e. $\forall \varepsilon>0, \exists N, \forall M>N, a_{M}>a-\varepsilon$; and (ii) for all $\varepsilon>0$, the sequence forever returns to being less than $a+\varepsilon$, i.e. $\forall \varepsilon>0, \forall N, \exists M>N, a_{M}<a+\varepsilon$. The requirements (i) and (ii) imply that such a number $a$ is unique; and if there is no such real number, we set lim inf $a_{n}=-\infty$. Similarly for lim sup. (One could summarize in topological jargon by saying that $\lim \inf a_{n}$ is the (possibly infinite) smallest of the sequence's accumulation points; and $\lim \sup a_{n}$ is the (possibly infinite) largest of its accumulation points.) So for any bounded set $X \subset \mathbb{R}^{n}$, we can define the lower and upper box dimension by

$$
\operatorname{dim}_{B}^{L}(X):=\lim _{\inf _{\delta \rightarrow 0}} \frac{\log N(\delta, X)}{-\log \delta} ; \operatorname{dim}_{B}^{U}(X):=\lim _{\sup _{\delta \rightarrow 0}} \frac{\log N(\delta, X)}{-\log \delta} ;
$$

and then we say that if these values are equal, that value is $X$ 's box dimension $=: \operatorname{dim}_{B}(X)$.

Again, a rich theory ensues (Falconer 2003, Chapter 3; Barnsley 1988, Chapter 5). For example: (i) familiar "regular" sets like points, lines and planes have box dimension equal to their topological dimension; (ii) for any set $X$, $\operatorname{dim}_{H}(X) \leq \operatorname{dim}_{B}^{L}(X) \leq \operatorname{dim}_{B}^{U}(X)$; and (iii) for a wide class of sets, the box and Hausdorff dimension are equal-but the box dimension has the advantage that it is often easier to calculate.

\subsection{The claims illustrated by emergent dimensions}

I turn to describing how the non-integral dimensions of Section 5.1 count as emergent behaviour in my sense, and how they illustrate my claims (1:Deduce), (2:Before) and (3:Herring) (listed in Section 1.2). As I announced, the illustrations do not need all the details, especially of Section 5.1.2. To keep things simple and brief, I specialize to sets like the Cantor set and Koch snowflake (Section 5.1.1) that are defined by taking a limit of an iterated process of definition. Then the illustrations unfold immediately, once we stipulate that having a non-integral dimension is to be the emergent property or behaviour: i.e. novel (or surprising) and robust, relative to a comparison class.

Certainly, non-integer dimensions are novel (more so than Section $4 \mathrm{~s}$ s limiting prob-

abilities). And they are 'robust' in at least two senses. First, the scaling dimension of 
Section 5.1.1 obviously takes the same value for congruent sets of points, and for enlarged and reduced versions of a given set: this invariance is a kind of robustness. Second and more interesting: as discussed in Section 5.1.2, there are various novel notions of dimension which can take non-integer values, and which are "cousins" of each other in various ways. They share the ideas of dimension as an exponent, and of taking successively finer covers or grids; and for wide classes of sets, their values agree. In particular, the values of Section 5.1.1 s scaling dimension are endorsed by Section 5.1.2 s Hausdorff and box dimension. So indeed it is fair to talk of 'emergent dimensions'.

\subsubsection{Emergence in the limit: with reduction - and without}

As to (1:Deduce): we have 'reduction as deduction' in as strong a sense as you could demand-provided we take the limit. The general situation is that at stage $N=0$, a "regular" set is given. Here "regular" can mean various things depending on the context, but I will take it to always imply having a well-defined topological dimension. Another set is then defined, yielding stage $N=1$, by a process that can be iterated to give sets at stages $N=2,3, \ldots$. At all finite stages, the defined sets are regular. And for a wide class of cases (including Section 5.1.1]s Cantor set $C$ and and Koch snowflake $K$ ), the stages' dimensions are all equal - and is the integer you would expect. For example, at stage $N$ for the Cantor set $C$, the defined set, $C_{N}$, is a union of closed sub-intervals of the unit interval; and its topological dimension is 1 , as you would expect. Similarly for the stages in defining $K$. But the "irregular" set is defined by taking the limit $N \rightarrow \infty$. In general it has a different topological dimension: thus $\operatorname{dim}(C)=0 \neq \operatorname{dim}\left(C_{N}\right) \equiv 1$. So topological dimension is not continuous in the limit; (footnote 3 notes how this shows discontinuous limits do not imply emergence). And more important for us: according to one or more of the novel notions of dimension (scaling, Hausdorff, box etc.), the set has a non-integral dimension. For example, $C$ 's dimension (according to all three notions) is about 0.63.

Thus the non-integral dimension, the emergent behaviour, is indeed deduced (and so reduced!) in the limit. In terms of my mnemonic notations: (1:Deduce) is illustrated as follows. Take as $T_{b}$ the theory of scaling dimension, and-or one or more of its generalizations like the Hausdorff or box dimension; and if you wish, include, as a sub-theory, the topological theory of dimension. Take as $T_{t}$ the assignments of non-integral dimensions to sets like $C, K$; (and if $T_{b}$ includes the generalizations, to other sets that are not exactly self-similar). Then clearly, we have reduction: $T_{b}$ contains $T_{t}$ ! (Or in terms of Section 3.3.2 s quantity $f$ whose value, 1 or 0 , records the presence or absence of the emergent property: $f_{\infty}=1$.)

But there is "the other side of the coin": the emergent behaviour is not deducible if we do not take the limit. Notice that the situation is a bit different from that for the method of arbitrary functions (Section 4.2.1). There, all one needed so as to deduce the emergent behaviour was consideration of the limit. Here, one needs ideas that go beyond the topological notion of dimension - discontinuous though it is, in the limits concerned. One needs the idea of dimension as an exponent, as developed in scaling dimension or its generalizations. But notwithstanding this difference, the main point is that (1:Deduce)'s second claim holds true again. Namely: if $T_{b}$ is just the traditional theory of dimension, 
there is no reduction; and because this weaker theory is salient, it is tempting to speak of irreducibility.

Finally, note another contrast with the method of arbitrary functions. Section 4.2.1 ended by noting that no roulette wheel has infinitely many arcs; nor is any wheel spun infinitely fast. In Section 3.1 s notation: there was no infinite system $\sigma(\infty)$. But in the fractals example, there are such infinite systems - the sets $C, K \subset \mathbb{R}^{n}$ etc.-and the whole discussion focusses on them.

\subsubsection{Emergence before the limit}

(2:Before) claims that before the limit, there is emergence in a weaker but still vivid sense. It is illustrated in a manner parallel to the method of arbitrary functions. Thus recall Section 4.2.2 $\mathrm{s}$ discussion of approximate equiprobability in, for example, a casino's roulette wheel. For fractals, the obvious analogue of the wheel is a computer running some software so as to produce a simulation of some fractal set, by iterating the steps of its definition some finite number $N$ of times. The most obvious case is computer graphics software, producing an approximate or coarse-grained image of a fractal set. Nowadays, such images are ubiquitous in films and games, superseding the static images in yesteryear's lavish books (e.g. Peitgen and Richter 1986).

It is easy to check that all of Section 4.2.2 $\mathrm{s}$ discussion-about how one can calculate, perhaps numerically, how closely a set-up approximates equiprobability, and how we philosophers can leave it to the casino-owners to worry about how close is close enough to be indiscernible by prospective gamblers - carries over to fractals, mutatis mutandis. I will save space by not spelling this out. In short: what was said there, about the practical purposes of a casino in making a wheel fair enough that a gambler cannot profit from assiduously observing its long-run statistics, carries over here to the practical purposes of a film studio in making a simulated image look fractal at spatial scales so small that even the most hawk-eyed cinema-goer cannot see that it is in fact not fractal.

But there are two other topics worth pausing over. One is obvious from the mention of computer graphics: the use of fractals to model naturally occurring objects like mountains, rocks, trees and leaves. This merits a separate discussion; cf. Section 5.3.

The other topic is an analogue for fractals of the quantifier-shift that Section 4.2.1 discussed as underlying the "two sides of the coin" in (1:Deduce). (This topic is also connected to the robustness requirement in my notion of emergence; but I will not pursue this.)

Thus take a traditional geometrical variable magnitude: in philosophers' jargon, a determinable property of a geometrical figure $F$. For example, consider 'contains a continuous arc of length greater than $L$ ' (variable $L$ ). And suppose we have an repeatable definitional process, that at its $M$ th iteration defines a figure (subset of $\mathbb{R}^{n}$ ), $F_{M}$, and that introduces successively finer structure so that for each value $L$ of the variable, $F_{M}$ lacks the property for sufficiently large $M$. That is: the property is lost after sufficiently many iterations. Or to put it more positively: an approximate or coarse-grained version 
of a fractal-like property is gained. For example, the definitional process might imply: $\forall L>0, \exists N, \forall M>N$ : the figure $F_{M}$ lacks arcs of length greater than $L$. But for smaller $L$, more iterations will be needed.

To make an analogy with Section 4.2.1 s quantifier-shift, we now develop this idea so as to both:

(a) use an 'resolution' $\varepsilon$, as is usual in definitions of convergence;

(b) make a "pointwise vs. uniform" contrast, by quantifying over some set $\mathcal{G}$ of geometrical properties, or sub-figures, of the figure $F_{M}$.

Thus suppose that in the figure $F_{M}$ at stage $M$, the only, or the largest, example of a property or sub-figure $G \in \mathcal{G}$ is of size (say, length) $L$. I will write this as: $\operatorname{Size}\left(F_{M}, G\right)=\varepsilon$. Then the successive loss of properties $G \in \mathcal{G}$-more exactly: the loss of visible, largeenough-to-be-seen, $G \in \mathcal{G}$-by a sequence of figures $\left\{F_{M}\right\}$ can happen: either pointwise across $\mathcal{G}$, viz.

$\forall \varepsilon, \forall G \in \mathcal{G}, \exists N \forall M>N: \operatorname{Size}\left(F_{M}, G\right)<\varepsilon ;$

or uniformly across $\mathcal{G}$, viz.

$\forall \varepsilon, \exists N \forall G \in \mathcal{G}, \forall M>N: \operatorname{Size}\left(F_{M}, G\right)<\varepsilon$.

Besides, there are alternatives to using such a set $\mathcal{G}$ so as to make the pointwise/uniform contrast. We could instead use different parts of the figures $F_{M}$. Thus one can imagine the stages of the definitional process to proceed at different "rates" in different regions: in different thirds, ninths,..., of the Cantor set; or sides, sub-sides, sub-sub-sides,..., of the Koch snowflake. If the rates vary in a suitably ever-slower way, across a denumerable sequence of sub-regions, one would get convergence to the fractal structure that is merely pointwise across the set.

\subsubsection{Supervenience is a red herring}

I shall be very brief about my third claim, (3:Herring): that although various supervenience theses are true, they yield little or no insight into emergence, or more generally, into "what is going on" in the example. For the situation is again like that for the method of arbitrary functions (Section 4.2.3): my claim holds true, essentially because supervenience makes no connection with the main ideas of the example - self-similarity and dimension as an exponent.

For any finite $N$, the property of interest, dimension, of the object concerned, i.e. of a subset $X \subset \mathbb{R}^{n}$, "supervenes on how $X$ is constituted from points" - in at least two obvious senses of this phrase. Namely: (i) the trivially strong sense in which only $X$ itself contains those very points (cf. set-theory's axiom of extensionality); (ii) the marginally weaker sense in which as regards its constitution from points, $X$ matches any congruent or scaled copy of $X$. And since in this example, there are infinite systems $\sigma(\infty)$, i.e. the "irregular" sets $C, K \subset \mathbb{R}^{n}$ etc., the same goes for $N=\infty$. That is: the dimension of these sets, in any of the several senses of dimension, thus supervenes.

But such supervenience theses are trivial and useless, for the two now-familiar reasons. (a): They provide no control on the infinity (infinite disjunction) they are concerned with, because no kind of limit is taken. (b): Their infinity makes no connection with the limit, 
$N \rightarrow \infty$, that the example is concerned with. In particular, the supervenience thesis gives no hint that we can use the idea of dimension as an exponent so as to define non-integral dimensions.

\subsection{The fractal geometry of nature?}

So far, the pure mathematics of dimension has dominated the discussion. But fractals have many empirical applications. As I discussed in Section 5.1.2. A, countless empirical studies have found power law behaviour with a dimension as a non-integral exponent: recall the examples of the coastline and electrodeposited copper. And Section 5.2.2 mentioned computer graphics' use of fractals to model objects like mountains, trees and leaves. This representational power of fractals is remarkable, indeed amazing 20 Thus fractals have been hailed as revealing the true geometry of nature, e.g. by Mandelbrot (1982). But this claim has been disputed (Shenker 1994, especially Sections 3-5; Smith 1998, pp. 31-38): hence this Subsection's title.

I will argue that with my claims (2:Before) and (4:Unreal), we can put this controversy to rest. I will distinguish two senses of the phrase 'geometry of nature', and propose that fractal geometry is a geometry of nature, in the second sense but not the first. It will be clear that (2:Before) corresponds to the second sense, while (4:Unreal) corresponds to the first. Finally, I will introduce an "abstract", rather than "natural history", sense of the phrase. In this last sense, fractal geometry is again a geometry of nature; and this again corresponds to (2:Before).

Suppose first that 'geometry of nature' means 'the completely accurate description of the shapes and sizes of macroscopic objects'. Then it sure looks like fractal geometry is the geometry of nature - as many a film with computer-generated graphics attests. But authors such as Shenker have objected that a fractal has an infinite sequence of intricate but similar structure on ever smaller length scales; while a mountain, rock, tree, fern and leaf do not, thanks to their atomic constitution. This objection is of course correct: recall my claim (4:Unreal) of Section 2, So despite initial appearances, fractal geometry is not in this sense the geometry of nature.

Indeed, the objection can be sharpened, in two ways: one theoretical, one practical. (Neither seems to have been noticed in the literature.) I touched on the theoretical sharpening, already in footnote 18, when I noted that while Euclidean geometry admits the similarity of triangles and other figures, on which self-similarity and so fractals depend, non-Euclidean geometries do not. This means that if physical space is in fact slightly nonEuclidean on even the tiniest scales, as general relativity and cosmology nowadays say, then macroscopic objects could not be exactly fractal - even if atomism was false and they

\footnotetext{
${ }^{20}$ And noticed by the wider culture: in Stoppard's play Arcadia (1993), the hero Valentine describes a stage-by-stage computer-simulation: 'If you knew the algorithm and fed it back say ten thousand times, each time there'd be a dot somewhere on the screen. You'd never know where to expect the next dot. But gradually you'd start to see this shape, because every dot would be inside the shape of this leaf. It wouldn't be a leaf, it would be a mathematical object.' In another passage he is lyrical about fractals' representation of other 'ordinary-sized stuff which is our lives, the things people write poetry about-clouds, daffodils, waterfalls'.
} 
were instead composed of continuous matter, even on arbitrarily small length scales. So here again, we meet my claim (4:Unreal) 21

The practical sharpening concerns the details of Section 5.1.2. A's empirical studies of power laws with a quantity $f$ proportional to a non-integral power of a resolution $\delta$ : $f=$ constant $\times \delta^{D}$. Suppose that faced with such a study, we ask: how many orders of magnitude of $\delta$ does the data report - or does the analysis in fact probe? The answer can be: disappointingly few. A survey of ninety-six Physical Review articles (in the years 1990-1996) reporting fractal analysis of data found that among these articles: (i) the average spread of resolutions that were probed was 1.3 orders of magnitude; and (ii) at most three orders of magnitude were probed (Avnir et al. 1998). In terms of measuring the length of a coastline: an "average paper" in the set surveyed by Avnir et al. would describe the coastline or its length as 'fractal', though the authors considered a spread of resolutions that went only from some length $L$ to about thirteen times $L, \approx 13 L$. And even the papers that were most stringent, or cautious, in describing their phenomenon as 'fractal' probed their resolution only up to three orders of magnitude 22

To sum up about this first sense of 'geometry of nature': if we ask the question,

Do fractals describe, with complete accuracy, the shapes and sizes of naturally occurring macroscopic objects?

we have to answer 'No'.

But despite this answer 'No', the representational power of fractals remains very striking. Power laws with a non-integral exponent describe very many phenomena; and our understanding of the phenomenon is often enhanced, empirically as well as theoretically, by adding to the bare power law, the suggestive language and exact theorems of fractal geometry. Here again we see that in a suitably weak sense, emergence can occur before the relevant limit: (2:Before) again!

Besides, this is consistent with (4:Unreal), since (2:Before) applies to values of the parameter $N$ which are typically much smaller than those making true (4:Unreal). That is: our 'No' answer turned upon our question's requiring complete accuracy. If instead we ask, in the context of modelling some specific phenomenon involving naturally occurring macroscopic objects, 'Do fractals describe, with sufficient accuracy, the shapes and sizes of these objects?', our answer would very often be 'Yes'. In this weaker sense, fractal geometry undoubtedly is a geometry of nature.

There is another aspect to this resolution of the controversy; (which, like the foregoing, should not be controversial!). So far I have considered the shapes and sizes of macroscopic

\footnotetext{
${ }^{21}$ I stress the phrases 'nowadays say' and 'macroscopic objects'. I of course agree that, for all we know, fractals may be involved as fundamental structures in the ultimate theory, at present unknown, of matter and-or space. But that is not our concern.

${ }^{22}$ Thanks to Leo Kadanoff for commenting that, happily, the range probed can also be much larger. He mentions the work of Libchaber and co-authors on turbulence, and Nagel and co-authors on glassy behaviour. Indeed, the former have probed five orders of magnitude (e.g. Castaing et al. 1989), and the latter have probed thirteen (Dixon et al. 1990). I presume that the latter group's Physical Review papers have been omitted from Avnir et al's survey for the ironic reason that they meritoriously avoid using the word 'fractal'.
} 
objects in physical space. But suppose we allow that 'geometry' applies to objects or structures in other spaces: in physical theories' state-spaces, which indeed often include objects and structures the theory calls 'geometric'. Some of these theories are strikingly successful, in the depth and accuracy of their theoretical descriptions and observational predictions. So some of these postulated geometric descriptions surely deserve to be called (a) 'geometry of nature': i.e. a geometry of an object or structure in a physically real space, albeit a more abstract (and often less visualizable) space than physical space. So if we ask instead a third question,

Do some of our successful physical theories use fractals to describe certain subsets of their abstract spaces, in particular attributing a non-integer dimension to such objects?

the answer is again: Yes.

Here are just two examples, out of many one could cite. One can check that in each of them, the justification for using fractals, i.e. for the $N \rightarrow \infty$ limit, is the Straightforward Justification of Section 3.3.3, with its two obvious reasons, mathematical convenience and empirical success.

(1): In classical mechanics, there are physically important fractal subsets of the phase spaces of systems. In particular, the famous Lorenz and Hénon attractors have fractal dimension. (The philosophical literature on chaos theory has discussed these, along the lines of the Straightforward Justification; e.g. Smith's (1998, pp. 41-43, 50-56) discussion of the Lorenz attractor.)

(2): Statistical mechanics describes some aspects (viz. critical points) of some processes (phase transitions, like boiling and freezing) with scale-free (regimes of) theories, which involve power-law behaviour on all scales and self-similarity, and therefore fractals. Section 7 will give more details. In particular, Section 7.2 .2 will discuss the phenomenon of cross-over: in which, as the parameter $N$ grows, the system's behaviour crosses over from illustrating (2:Before), at intermediate values of $N$, to illustrating (4:Unreal), at larger values of $N$, to again illustrating (2:Before) at yet larger values of $N$. Thus crossover will be a vivid illustration of my swings-and-roundabouts, (2:Before)-and-(4:Unreal), Yes-and-No, answer to the question 'Is fractal geometry the geometry of nature?'

\subsection{The story so far: summing up fractals}

Let me sum up the fractals example as a list of six morals. It will be obvious, without making explicit my four claims, or Section 3.3.3 s Straightforward Justification, or the parallels with the method of arbitrary functions, that this list also sums up the whole story so far.

(i): The large finite is often well-modelled by the infinite.

(ii): Such models are often justified in a straightforward, even obvious, way, by mathematical convenience and empirical success.

(iii): The infinite often brings new mathematical structure: in the fractals example, non-integer dimension. 
(iv): Nevertheless, there is often a reduction: the emergent non-integer dimensions are reducible to a sufficiently rich theory that takes the infinite limit.

(v): On the other hand, one can often see emergent behaviour on the way to the limit. Thus in the fractals example: the larger i.e. worse the spatial resolution - the worse your eyesight - the sooner in the iterative definitional process you see (more precisely: think you see!) the fractal structure.

(vi): Various supervenience theses hold-but they are trivial, or at least scientifically useless.

\section{Superselection}

\subsection{Introduction}

I turn to the first of my two examples from physics proper: superselection in the $N \rightarrow \infty$ limit of quantum mechanics. As in the previous two examples, I will first expound the technical details without reference to my claims (Section 6.2), and then illustrate the claims (Section 6.3). But to make those illustrations richer, I will give more details than previously. So the aim of this Subsection is to describe the lie of the land - and so indicate which details matter more. I will do this by describing (i) the general topic of the quantum-classical transition (Section 6.1.1) and (ii) the basic idea of superselection in the limit (Section 6.1.2).

\subsubsection{Out of the quantum soup}

This example is an aspect of a much larger topic: the emergence of the classical world from the quantum world. This is often discussed in terms of a limit $\hbar \rightarrow 0$. But the topic involves a lot else, such as proposals for the importance of specific states (e.g. coherent states), and-or of specific physical processes (e.g. decoherence). My example is just one case of the general idea that classical physics should emerge for "large" quantum systems: so in this example of my theme that $N \rightarrow \infty, N$ will be the number of degrees of freedom. (We will see how this $N \rightarrow \infty$ relates to $\hbar \rightarrow 0$. But as I announced at the end of Section 2. I will not in this Section pursue my fourth claim (4:Unreal).)

By way of glimpsing the larger topic, I note that there are now many well-understood examples of classical physics emerging for large (i.e. $N \rightarrow \infty$ ) quantum systems. Much of this work uses the algebraic approach to quantum theory, in which systems are primarily described by algebras of quantities, on which the states are linear expectation functionals; and I shall follow suit. For example, Sewell's recent monograph uses this approach to articulate a 'rather general scheme for ... deriving the irreversible deterministic macroscopic dynamical laws of many-particle systems, such as those of hydrodynamics or heat conduction, from their underlying quantum dynamics' (2002, p. 87). This scheme forms a girder across the rest of Sewell's book: it is realised in detail, in several examples 23

\footnotetext{
${ }^{23}$ The first is a toy-model reminiscent of quantum Brownian motion: a massive particle at one end of
} 
To illustrate my claims, I can treat superselection more simply than by following Sewell's scheme: the main simplification will lie in ignoring dynamics (especially, the deduction of classical equations of motion) and aiming only to deduce, as $N \rightarrow \infty$, classical kinematics (more precisely, commutativity of quantities). But to give a general perspective, it is worth first quoting Sewell's scheme. (These details are not needed later.)

Sewell's macroscopic picture is given by a classical dynamical system $\mathcal{M}=(\mathcal{Y}, T)$ where $\mathcal{Y}$ is a topological space, and $\left\{T(t) \mid t \in \mathbb{R}_{+}\right\}$is a one-parameter semigroup of transformations of $\mathcal{Y} . \mathcal{M}$ is to correspond to the dynamics of a one-parameter family $Y_{\Omega}$ of finite sets of quantities of a quantum system $\Sigma$; where $\Sigma$ 's evolution will be given by a one-parameter group $\alpha(\mathbb{R})$ of automorphisms of $\Sigma$ 's algebra of quantities. So we write $Y_{\Omega}=\left\{Y_{\Omega}^{(1)}, \ldots, Y_{\Omega}^{(k)}\right\}$

Here $\Omega$ is a "large", dimensionless, positive parameter whose magnitude provides a measure of the quantities' macroscopicality. We then require that there is a set $\Delta$ of states of $\Sigma$ such that:

(a): For each state $\phi \in \Delta$, the means and dispersions of $Y_{\Omega}$ converge to limits, $Y(\phi) \equiv Y$ and 0 , respectively, as $\Omega \rightarrow \infty$.

(b): As $\phi$ runs through $\Delta$, the resultant range of the limiting values $Y \equiv Y(\phi)$ is just the classical phase space $\mathcal{Y}$. (So $\mathcal{Y}$ is a subset of $\mathbb{R}^{k}$.)

(c) The classical dynamical semigroup $T\left(\mathbb{R}_{+}\right)$of $\mathcal{M}$ is induced by the evolution of $\Sigma$ from states in $\Delta$, on a "macroscopic" time scale $\Omega^{\gamma}$, with $\gamma>0$. To be precise: we require that the mean and dispersion, for a state $\phi \in \Delta$, of the $k$ macroscopically time-evolved quantities $\alpha\left(\Omega^{\gamma} t\right) Y_{\Omega}$ should converge to $T(t) Y$ and 0 , respectively, as $\Omega \rightarrow \infty$.

Accordingly, Section 6.2 will give analogues of Sewell's kinematic (a) and (b), though not of his dynamical (c). But in two other respects, I will go beyond the above scheme. First, I will emphasise that there are two different infinite limits to be considered:

(i) the classical limit of ever-larger (increasing $N$ ) quantum systems, which will have all quantities commuting, and which corresponds to Sewell's (a) and (b); and

(ii) the quantum limit of ever-larger quantum systems, which will exhibit superselection, i.e. some quantities apart from multiples of the identity operator commuting with all quantities: in algebraic terms, the centre of the algebra of quantities being non-trivial.

Second, I will discuss these limits in terms of deformation quantization: which has the merits not only of generality and precision, but also of showing how (both of) these limits are continuous - pace the frequent emphasis on the singularity of $\hbar \rightarrow 0$, and philosophers' frequent emphasis on discontinuous limits as a signature of emergence. (Recall the discussions in Sections 1.1 and 3.3.) In both these respects, my treatment follows (but simplifies) some material in Landsman's masterly account of the quantum-classical transition (2006).

a semi-infinite chain of much lighter particles, with harmonic nearest-neighbour interactions; p. 94-106. His Chapters 7, 10 and 11 describe much more advanced cases, e.g. lasers. 


\subsubsection{The idea of superselection in the limit}

Setting aside all details of both mathematics and physics, I will state in a nutshell the idea of superselection emerging in the limit $N \rightarrow \infty$, by using the idea that a sequence of numbers, each less than one, has in general an infinite product equal to zero. Thinking of each number as an inner product of two vectors allows us to think of this zero as orthogonality: a hallmark of superselection.

Imagine that we assign two real vectors $\in \mathbb{R}^{3}$, each of unit-length, with angle $\theta$ between them, the "score" $\cos \theta$. So if they are parallel, the score is $\cos 0=1$; but if they are not parallel, it is less than 1. And suppose we assign two sequences of unit vectors, each with $N$ members, a score that is the product of the cosines of the angles between corresponding members:

$$
\begin{array}{r}
\operatorname{score}\left(<v_{1}, v_{2}, \ldots, v_{N}>,<u_{1}, u_{2}, \ldots, u_{N}>\right):= \\
\cos \theta_{v_{1} u_{1}} \cos \theta_{v_{2} u_{2}} \ldots \cos \theta_{v_{N} u_{N}}
\end{array}
$$

We now let $N$ tend to infinity, and consider the limiting values of the score we have defined. We note two sorts of case:-

(1): A pair of infinite sequences $\left\langle v_{i}\right\rangle,<u_{i}>$ in which the vectors at corresponding positions $i$ are not parallel, only for finitely many $i$. Then only finitely many factors in the score will be different from 1; infinitely many factors will be 1 . So the total infinite product of numbers is a product of finitely many cosines each less than 1 . This is some real number of modulus less than 1 . It might be zero: namely if at least one pair of vector $v_{i}, u_{i}$ are perpendicular.

(2): A pair of infinite sequences $\left\langle v_{i}\right\rangle,<u_{i}>$ in which the vectors at corresponding $i$ are not parallel, for infinitely many $i$. So the total infinite product of numbers is a product including infinitely many numbers that are each less than 1 . In general, this infinite product is zero (and even if there are also infinitely many factors each equal to $1)$.

These elementary considerations underly the emergence of superselection in the $N \rightarrow$ $\infty$ limit of quantum mechanics. (1) corresponds to two quantum states (two infinite sequences of unit-vectors) being in the same superselection sector. And (2) corresponds to two quantum states being in different superselection sectors.

Now I enter in to details. As in my previous two examples, I first expound the technicalities without reference to my claims (Section 6.2), and then illustrate the claims (Section 6.3).

\subsection{Superselection in the $N \rightarrow \infty$ limit of quantum mechanics}

In Section 6.2.1, I describe our prototype systems: finite and infinite spin chains. This will already exhibit the idea just expounded, of a "score" of sequences of vectors that converges to zero. In Section 6.2.2, I introduce the ideas of deformation quantization (especially continuous fields of algebras of quantities), in terms of which our $N \rightarrow \infty$ limits will be continuous. In Section 6.2.3, I treat the classical infinite- $N$ limit of quantum systems: 
for spin chains, this means identifying macroscopic quantities with averages of quantum observables, with the average being taken over greater and greater segments of the chain. In Section 6.2.4, I treat the quantum infinite- $N$ limit: here the limiting quantities are local in the sense that they "ignore" all but a finite part of the system. In Section 6.2.5, I discuss classical states of the quantum-infinite, and thus connect with superselection. NB: Each Section begins with a brief statement and then, after the announcement 'In Detail', gives details - which the reader in a hurry can skip.

For all this material, and much more, I recommend Landsman (2006, especially Sections 4.3, 6.1-6.4), who also gives many references (and whose notation I adopt). Compared with Landsman, I have chosen to down-play infinite tensor products and the representation of algebras, and to emphasise deformation quantization. This avoids repeating material which is well-known in the philosophy of physics literature; and more important, gives what we need for Section 6.3] s illustrations of my claims.

\subsubsection{Spin chains}

I begin with three claims about spin chains, which serve as ideal infinite models of ferromagnets. I shall take a doubly infinite spin-half chain, with sites labelled by the integers Z. But it will be clear that similar claims would hold for higher spin, and for a onedimensional half-infinite chain (sites labelled by $\mathbf{N}$ ) or for a two- or three-dimensional spin lattice $\left(\mathbf{Z}^{2}\right.$ or $\left.\mathbf{Z}^{3}\right)$. In these claims, we eschew infinite tensor products and non-separable Hilbert spaces. Rather we define a continuous family of separable Hilbert spaces, each of which will later turn out to be a superselection sector.

The overall physical idea is that:

(a) The vacuum i.e. ground state has all the spins aligned in one spatial direction, with other (higher-energy) states built up by flipping a finite number of spins from the preferred direction (and superposing); yielding a separable Hilbert space representing the spin-algebra.

(b) But nature does not prefer one such direction. So for any direction, there is a vacuum state with the spins thus aligned; and the higher-energy states built up from this vacuum yield an associated Hilbert space.

(c) These Hilbert space representations differ in a global/macroscopic quantity (spin density).

(d) The representations are thereby unitarily inequivalent - even though intuitively, a global rotation (an element of $\mathrm{SO}(3)$ ) of course rotates one vacuum to another.

In Detail: One shows four claims, e.g. for the one-dimensional doubly infinite spin chain; (cf. e.g. Sewell 1986: 16-18, or 2002: 15-18).

(a): For each direction (where positive and negative $z$-directions count as two directions), there is an irreducible representation of the infinite spin algebra generated by denumerably many pairwise-commuting copies of the trio of the Pauli matrices, i.e. generated by matrices: $\left\{\sigma_{\mathbf{n}}:=\left(\sigma_{n, x}, \sigma_{n, y}, \sigma_{n, z}\right) \mid n=0, \pm 1, \pm 2, \ldots\right\}$.

Let $S$ be the set of doubly infinite $(+1,-1)$-sequences, $s=\left(s_{n}\right)_{n \in \mathbb{Z}}, s_{n}= \pm 1$. We 
think of this as the set of "classical" configurations of the eigenvalues \pm 1 of $\sigma_{z}$ at each site. Let $S^{(+)} \subset S$ be those configurations $s$ with all but finitely many $s_{n}=+1$. We think of these as local modifications of the $z$-up (classical) vacuum $s^{(+)}=(. ., 1, \ldots)$. Let $\mathcal{H}^{(+)}$ be the square-summable functions on $S^{(+)}$:

$$
\mathcal{H}^{(+)}:=\left\{\phi:\left.S^{(+)} \rightarrow \mathbb{C}\left|\Sigma_{s \in S^{(+)}}\right| \phi(s)\right|^{2}<\infty\right\}
$$

with inner product

$$
\langle\phi, \psi\rangle^{(+)}:=\Sigma_{s \in S^{(+)}} \bar{\phi}(s) \psi(s) .
$$

The vectors $\phi_{s}^{(+)}, s \in S^{(+)}$defined by $\phi_{s}^{(+)}\left(s^{\prime}\right)=\delta_{s, s^{\prime}}, s, s^{\prime} \in S^{(+)}$, are in one-one correspondence with the configurations $s$. They form an orthonormal basis of $H^{(+)}$.

We can now define operators whose action on the basis vectors $\phi_{s}^{(+)}$is the analogue of the action of the three Pauli matrices on a single spin. That is, we define $\left\{\sigma_{n, u}^{(+)} \mid n \in\right.$ $\mathbb{Z}, u=x, y, z\}$ on $\mathcal{H}^{(+)}$in the obvious way, so as to build a representation on $\mathcal{H}^{(+)}$of the abstract spin-algebra:

$$
\left[\sigma_{n, x}, \sigma_{n, y}\right]=2 i \sigma_{n, z} \text { etc. ; }\left[\sigma_{m, x}, \sigma_{n, y}\right]=0 \text { for } m \neq n \text { etc. }
$$

The representation is irreducible since we can pass from any basis vector $\phi_{s}^{(+)}$to any other $\phi_{s^{\prime}}^{(+)}$by a sequence of operators $\sigma_{n, u}^{(+)}$.

Note that because we fixed on the denumerable $S^{(+)} \subset S$, not the continuously large $S$, we got a separable Hilbert space $\mathcal{H}^{(+)}$. So this representation does not requires us to make sense of a denumerable tensor product (of copies of $\mathbb{C}^{2}$ ).

(b): We can "play the same game", starting with $z$-down. That is: let $S^{(-)} \subset S$ be those configurations $s$ with all but finitely many $s_{n}=-1$. We think of these as local modifications of the $z$-down (classical) vacuum. We get a representation on $\mathcal{H}^{(-)}$.

Of course, the choice of $z$ was arbitrary. So we can build a representation for any direction. And each such representation: (a) takes the ground state to have all spins aligned along the direction; and (b) builds elements of the representation as all linear combinations of product states obtained from the ground state by a finite number of unitary transformations (spin-flips or rotations) at individual sites.

(c): For each such representation, every state in it (indeed every density matrix on it) has a common value of a global quantity, (aka: classical or macroscopic quantity): namely, the (vector) spin density defined as the limit as $N \rightarrow \infty$ of the average of the spin matrices at the sites $-N,-N+1, \ldots, N-1, N$, i.e. $\lim _{N \rightarrow \infty} \frac{1}{2 N+1} \Sigma_{n=-N}^{+N} \sigma_{\mathbf{n}}$.

In more detail: On our first space $\mathcal{H}^{(+)}$, define

$$
\mathbf{m}_{N}^{(+)}:=\frac{1}{2 N+1} \Sigma_{n=-N}^{+N} \sigma_{\mathbf{n}}^{(+)}
$$

so that the expectation value on basis states is

$$
\left\langle\phi_{s}^{(+)}, \mathbf{m}_{N}^{(+)} \phi_{s}^{(+)}\right\rangle=\left(0,0, \frac{1}{2 N+1} \Sigma_{n=-N}^{+N} s_{n}\right)
$$


Since all but a finite number of $s_{n}$ are $+1,6.6$ implies, with $\mathbf{k}$ the unit vector along $0 z$ :

$$
\lim _{N \rightarrow \infty}\left\langle\phi_{s}^{(+)}, \mathbf{m}_{N}^{(+)} \phi_{s}^{(+)}\right\rangle=\mathbf{k}, \forall s \in S^{(+)} .
$$

Similarly, one has:

$$
\lim _{N \rightarrow \infty}\left\langle\phi_{s}^{(+)}, \mathbf{m}_{N}^{(+)} \phi_{s^{\prime}}^{(+)}\right\rangle=0, \text { for } s \neq s^{\prime}:
$$

for in the $z$-component, $\mathbf{m}_{N}^{(+)}$gives no spin-flip or rotation, so that the orthogonality, $\left\langle\phi_{s}^{(+)}, \phi_{s^{\prime}}^{(+)}\right\rangle=0$, yields 0 ; while in the $x$ and $y$ components, the eventual agreement of $s$ and $s^{\prime}$ in having thereafter always the value +1 means that $\mathbf{m}_{N}^{(+)}$'s spin-flips and rotations give inner product zero at those sites, while the increasing $2 N+1$ denominator kills any initial non-zero contribution got from $\mathbf{m}_{N}^{(+)}$'s action. Thus from 6.6] and 6.8, it follows that for any unit vector $\Psi^{(+)} \in \mathcal{H}^{(+)}$,

$$
\lim _{N \rightarrow \infty}\left\langle\Psi^{(+)}, \mathbf{m}_{N}^{(+)} \Psi^{(+)}\right\rangle=\mathbf{k}
$$

So this limiting spin density is a global property of the representation. And the representations built from other vacua will have different unit vectors in $\mathbb{R}^{3}$ as their limiting spin densities.

As we will discuss in more detail: these are the system's superselection sectors. There are continuously many sectors, each of denumerable dimension.

(d): Each such representation is unitarily inequivalent to every other. For example: if the representations on $\mathcal{H}^{(+)}$and $\mathcal{H}^{(-)}$were unitarily equivalent, there would be a unitary $U: \mathcal{H}^{(+)} \rightarrow H^{(-)}$with $U \sigma_{\mathbf{n}}^{(+)} U^{-1}=\sigma_{\mathbf{n}}^{(-)}$. This would imply that $U \mathbf{m}_{N}^{(+)} U^{-1}=\mathbf{m}_{N}^{(-)}$. This in turn would imply that, for any unit vectors $\Psi^{( \pm)} \in \mathcal{H}^{( \pm)}$with $\Psi^{(+)}=U^{-1} \Psi^{(-)}$

$$
\left\langle\Psi^{(+)}, \mathbf{m}_{N}^{(+)} \Psi^{(+)}\right\rangle=\left\langle\Psi^{(-)}, \mathbf{m}_{N}^{(-)} \Psi^{(-)}\right\rangle:
$$

but this must be false, since the two sides have different limits, viz. $\pm \mathbf{k}$, as $N \rightarrow \infty$.

\subsubsection{Continuous fields of algebras and deformation quantization}

The main idea we need from the modern theory of deformation quantization (in the $C^{*}$-algebraic approach) is the idea of a continuous field of algebras of quantities. For Sections 6.2.3 and 6.2.4 will define such fields in such a way that their $N \rightarrow \infty$ limits are continuous. But when I enter details, I will also define two other central ideas of the theory.

The idea is that a continuous field of algebras of quantities is like a bundle in differential geometry. The base space is a set $I \ni \hbar$ of real numbers, and the fibre above each point $\hbar \in I$ is a $C^{*}$-algebra $\mathcal{A}_{\hbar}$ representing the system's quantities for that value of $\hbar$. (Of course, this is not meant to suggest that the value of $\hbar$ really varies. As usual, this is shorthand, essentially for the ratio of $\hbar$ to the typical values of the system's, or problem's, actions.) The topology of the bundle is defined indirectly by specifying what are its continuous sections. As we will see: the value $\hbar=0$ can correspond to a $N \rightarrow \infty$ limit; 
and we can choose the algebras (and the definitions of continuous sections) so as to make this either a classical limit or a quantum limit.

More formally: a continuous field of $C^{*}$-algebras over $I \ni \hbar$ consists of a $C^{*}$-algebra $\mathcal{A}$, and a collection of $C^{*}$-algebras $\left\{\mathcal{A}_{\hbar}\right\}_{\hbar \in I}$, subject to certain conditions which imply that:

(i): the family $\left(\mathcal{A}_{\hbar}\right)_{\hbar \in I}$ of $C^{*}$-algebras is glued together by specifying the space of continuous sections of the bundle $\coprod_{\hbar \in I} \mathcal{A}_{\hbar}$ (where $\coprod$ indicates disjoint union);

(ii): the $C^{*}$-algebra $\mathcal{A}$ can be identified with this space of sections.

In Detail: A continuous field of $C^{*}$-algebras over $I \ni \hbar$ consists of a $C^{*}$-algebra $\mathcal{A}$, a collection of $C^{*}$-algebras $\left\{\mathcal{A}_{\hbar}\right\}_{\hbar \in I}$, and a surjective morphism $\varphi_{\hbar}: \mathcal{A} \rightarrow \mathcal{A}_{\hbar}$ for each $\hbar \in I$ such that:

(i): the function $\hbar \mapsto\left\|\varphi_{\hbar}(A)\right\|_{\hbar}$ is in $C_{0}(I)$ for all $A \in \mathcal{A}$

(ii): the norm of any $A \in \mathcal{A}$ is $\|A\|=\sup _{\hbar \in I}\left\|\varphi_{\hbar}(A)\right\|$;

(iii): for any $f \in C_{0}(I)$ and $A \in \mathcal{A}$ there is an element $f A \in \mathcal{A}$ for which $\varphi_{\hbar}(f A)=$ $f(\hbar) \varphi_{\hbar}(A)$ for all $\hbar \in I$.

The idea is that the $C^{*}$-algebras $\left(\mathcal{A}_{\hbar}\right)_{\hbar \in I}$ are glued together by a topology on the disjoint union $\coprod_{\hbar \in[0,1]} \mathcal{A}_{\hbar}$. But this topology is defined indirectly, by specifying the space of continuous sections of the "bundle". Namely, a continuous section of the field is defined to be a map $\hbar \mapsto A_{\hbar}$ where $A_{\hbar} \in \mathcal{A}_{\hbar}$ for which there is an $A \in \mathcal{A}$ such that $A_{\hbar}=\varphi_{\hbar}(A)$ for all $\hbar \in I$. So the $C^{*}$-algebra $\mathcal{A}$ may actually be identified with the space of continuous sections of the field: if we do so, the morphism $\varphi_{\hbar}$ is just the evaluation map at $\hbar$.

With this idea of a continuous field of algebras, we can define a deformation quantization. We begin with a classical phase space $M$, on which the continuous complex functions $f$ represent quantities (through their real and imaginary parts). We want to define, for each value of $\hbar$, a quantization map $\mathcal{Q}_{\hbar}$ mapping such functions $f$ to elements of $\mathcal{A}_{\hbar}$, subject to various conditions - in particular, the "Dirac" condition that the Poisson bracket on $M$ is mapped to the quantum mechanical commutator (times $\frac{i}{\hbar}$ ). Two technical comments: (i) $M$ can be a Poisson manifold - a mild generalization of the usual symplectic manifold; (ii) on the other hand, we take $f$ to be smooth with compact support - the space $C_{c}^{\infty}(M)$ of these functions is a norm-dense sub-algebra of $C_{0}(M)$.

Thus we define: a deformation quantization of a phase space $M$ is a continuous field of $C^{*}$-algebras $\left(\mathcal{A}_{\hbar}\right)_{\hbar \in[0,1]}$ (with $\mathcal{A}_{0}=C_{0}(M)$ ), along with a family of linear maps $\mathcal{Q}_{\hbar}$ : $C_{c}^{\infty}(M) \rightarrow \mathcal{A}_{\hbar}, \hbar \in(0,1]$, that are self-adjoint (i.e. $\left.\mathcal{Q}_{\hbar}(\bar{f})=\mathcal{Q}_{\hbar}(f)^{*}\right)$, and such that:

(i) for each $f \in C_{c}^{\infty}(M)$ the map defined by $0 \mapsto f$ and $\hbar \mapsto \mathcal{Q}_{\hbar}(f)(\hbar \neq 0)$ is a continuous section of the given continuous field of algebras;

(ii) for all $f, g \in C_{c}^{\infty}(M)$ one has the "Dirac" condition

$$
\lim _{\hbar \rightarrow 0}\left\|\frac{i}{\hbar}\left[\mathcal{Q}_{\hbar}(f), \mathcal{Q}_{\hbar}(g)\right]-\mathcal{Q}_{\hbar}(\{f, g\})\right\|_{\hbar}=0 .
$$

\footnotetext{
${ }^{24}$ Here \|\|$_{\hbar}$ is the norm in $\mathcal{A}_{\hbar}$, and $C_{0}(I)$ is the continuous complex functions on $I$ that vanish at infinity in the usual sense that for any $\varepsilon>0$, there is a compact set beyond which the function is less than $\varepsilon$. For any locally compact Hausdorff space $X, C_{0}(X)$ is a $C^{*}$-algebra when equipped with the supremum norm, $\|f\|_{\infty}:=\sup _{x \in X}|f(x)|$. In any case, for our examples $I$ is itself compact.
} 
This definition turns out to imply other natural "meshing" conditions, such as:

$$
\lim _{\hbar \rightarrow 0}\left\|\mathcal{Q}_{\hbar}(f) \mathcal{Q}_{\hbar}(g)-\mathcal{Q}_{\hbar}(f g)\right\|_{\hbar}=0 ; \lim _{\hbar \rightarrow 0}\left\|\mathcal{Q}_{\hbar}(f)\right\|_{\hbar}=\|f\|_{\infty}
$$

where $\|f\|_{\infty}$ is the supremum norm $\sup _{z \in M}|f(z)|$ on $\mathcal{A}_{0}=C_{0}(M)$.

By way of indicating the power of deformation quantization, let me sketch how a definition which it enables, viz. of a continuous field of states (treated as linear functionals on quantities), gives a natural generalization of the notion of coherent states - which are the focus of many discussions of the $\hbar \rightarrow 0$ limit of quantum mechanics. (The details of Sections 6.2.5 and 6.3.1 will also need this definition.)

Given a state $\omega_{\hbar}$ on $\mathcal{A}_{\hbar}$ for each $\hbar \in[0,1]$ : we define the family of states to be a continuous field (relative to a given deformation quantization), whenever the function $\hbar \mapsto \omega_{\hbar}\left(\mathcal{Q}_{\hbar}(f)\right)$ is continuous on $[0,1]$ for each $f \in C_{c}^{\infty}(M)$. (In fact, this notion is intrinsically defined by the continuous field of $C^{*}$-algebras, and is therefore independent of the quantization maps $\mathcal{Q}_{\hbar}$.) In particular, one has

$$
\lim _{\hbar \rightarrow 0} \omega_{\hbar}\left(\mathcal{Q}_{\hbar}(f)\right)=\omega_{0}(f) .
$$

Now recall the idea of a sequence of coherent states $\left(\Psi_{z}^{\hbar}\right)_{\hbar \in[0,1]}$ that tend, as $\hbar \rightarrow 0$, to be ever more peaked about the classical phase space state $z \in M$, so that in the limit, the quantum expectation value tends to the value of the classical quantity at the state $z$ :

$$
\lim _{\hbar \rightarrow 0}\left(\Psi_{z}^{\hbar}, \mathcal{Q}_{\hbar}(f) \Psi_{z}^{\hbar}\right)=f(z)
$$

(This of course exemplifies (a) and (b) in Sewell's scheme, reported in Section 6.1.1; the coherent states are Sewell's set $\Delta$ of quantum states, $\hbar$ is the reciprocal of his $\Omega$, and $M$ is his phase space $\mathcal{Y}$.) Clearly, eq. 6.13 generalizes eq. 6.14. Indeed, one can show that coherent states are examples of continuous fields of (pure) states; (for details, cf. Landsman (2006, Section 4.2, 5.1).

\subsubsection{The classical infinite: macroscopic quantities from symmetric sequences}

I turn to the classical $N \rightarrow \infty$ limit of $N$ copies of a quantum system that has an algebra of quantities $\mathcal{A}_{1}$. The idea is to identify a classical, macroscopic quantity with a limiting average of a microscopic quantum quantity. Thus the microscopic quantity is defined over say $M$ copies of the system; (so it is often called an " $M$-particle observable"). But this quantity is averaged over $N$ copies $(N>M)$, and then we take the limit $N \rightarrow \infty$. For spin chains (Section 6.2.1), this means the average is taken over greater and greater segments of the chain.

This is made precise in terms of a continuous field of $C^{*}$-algebras $\mathcal{A}^{(c)}$ over different values of $N$. The four main points are:-

(i): To conform to Section 6.2.2 s notation for $\hbar \rightarrow 0$, we in fact use the reciprocal of $N, 1 / N$, rather than $N$. Thus $\left.\mathcal{A}_{1 / N}^{(} c\right)$ will be the usual algebra of quantities for $N$ 
copies of the basic quantum system, viz. $\hat{\otimes}^{N} \mathcal{A}_{1}$, i.e. the $N$-fold tensor power of the basic algebra $\mathcal{A}_{1}$.

(ii): We associate a macroscopic quantity with a sequence $A=\left(A_{1}, A_{2}, \cdots\right)$ of algebra-

elements, with $A_{N} \in \hat{\otimes}^{N} \mathcal{A}_{1}=: \mathcal{A}_{1}^{N}$, that is symmetric in the sense that its tail consists of some finite-particle quantity averaged over an ever-larger number of systems or sites. That is: a sequence is symmetric if each of its elements $A_{P}$ for all $P$ greater than some fixed $N$ consists of some quantity on $M$ (with $M<N$ ) copies of the system (an " $M$ particle observable") averaged over $P$ copies.

(iii): In the limit $N \rightarrow \infty$, symmetric sequences commute; and this will mean that the macroscopic quantities form a commutative $C^{*}$-algebra. In fact this algebra is isomorphic to $C\left(\mathcal{S}\left(\mathcal{A}_{1}\right)\right.$ ), i.e. the continuous complex functions on the quantum state-space for (the density matrices on) the basic algebra $\mathcal{A}_{1}$.

(iv): The important features (both here and in subsequent Sections) are present in the construction for the simplest basic algebra $\mathcal{A}_{1}:=M_{2}(\mathbb{C})$, i.e. a spin chain with a spinhalf system at each site. So we can throughout the discussion keep this system in mind. For example, $\mathcal{S}\left(M_{2}(\mathbb{C})\right)$ is the Bloch sphere $B^{3}$ in $\mathbb{R}^{3}$, with pure states on the boundary $\partial B^{3}=S^{2}$. So according to (iii), the macroscopic quantities for a chain of spin-halves are given by the continuous complex functions on the Bloch sphere.

In Detail: From $\mathcal{A}_{1}=$ say $M_{2}(\mathbb{C})$, we will construct a continuous field of $C^{*}$-algebras $\mathcal{A}^{(c)}$ over the discrete set

$$
I=0 \cup 1 / \mathbb{N}=\left\{0, \ldots, 1 / N, \ldots, \frac{1}{3}, \frac{1}{2}, 1\right\} \subset[0,1],
$$

by putting

$$
\begin{array}{r}
\mathcal{A}_{0}^{(c)}:=C\left(\mathcal{S}\left(\mathcal{A}_{1}\right)\right) \\
\mathcal{A}_{1 / N}^{(c)}:=\mathcal{A}_{1}^{N}:=\hat{\otimes}^{N} \mathcal{A}_{1} .
\end{array}
$$

Thus if $\mathcal{A}_{1}=M_{2}(\mathbb{C})$, then $\mathcal{S}\left(M_{2}(\mathbb{C})\right)$ is the Bloch sphere $B^{3}$ in $\mathbb{R}^{3}$, and $\mathcal{A}_{0}^{(c)}$ is the set of continuous functions on $B^{3}$.

To define symmetric sequences, we first say that the symmetrization operator $S_{N}$ : $\mathcal{A}_{1}^{N} \rightarrow \mathcal{A}_{1}^{N}$ is given by (linear and continuous) extension of

$$
S_{N}\left(B_{1} \otimes \cdots \otimes B_{N}\right):=\frac{1}{N !} \sum_{\sigma \in \mathfrak{S}_{N}} B_{\sigma(1)} \otimes \cdots \otimes B_{\sigma(N)},
$$

where $\mathfrak{S}_{N}$ is the permutation group on $N$ elements and $B_{i} \in \mathcal{A}_{1}$ for all $i=1, \ldots, N$. Then we define Symmetrization maps $j_{N M}: \mathcal{A}_{1}^{M} \rightarrow \mathcal{A}_{1}^{N}$ by

$$
j_{N M}\left(A_{M}\right)=S_{N}\left(A_{M} \otimes 1 \otimes \cdots \otimes 1\right)
$$

with $N-M$ copies of $1 \in \mathcal{A}_{1}$ so as to get an element of $\mathcal{A}_{1}^{N}$. For example, $j_{N 1}: \mathcal{A}_{1} \rightarrow \mathcal{A}_{1}^{N}$ is given by

$$
j_{N 1}(B)=\bar{B}^{(N)}=\frac{1}{N} \sum_{k=1}^{N} 1 \otimes \cdots \otimes B_{(k)} \otimes 1 \cdots \otimes 1
$$


where $B_{(k)}$ is $B$ as an element of the $k$ 'th copy of $\mathcal{A}_{1}$ within $\mathcal{A}_{1}^{N}$. As $\bar{B}^{(N)}$ indicates, this is the 'average' of $B$ over all copies of $\mathcal{A}_{1}$. More generally, in forming $j_{N M}\left(A_{M}\right)$ an operator $A_{M} \in \mathcal{A}_{1}^{M}$ that involves $M$ sites is averaged over $N \geq M$ sites. When $N \rightarrow \infty$ this means that one forms a macroscopic average of an $M$-particle operator.

A sequence $A=\left(A_{1}, A_{2}, \cdots\right)$ of algebra elements with $A_{N} \in \mathcal{A}_{1}^{N}$ is symmetric when

$$
A_{N}=j_{N M}\left(A_{M}\right)
$$

for some fixed $M$ and all $N \geq M$. So the tail of a symmetric sequence consists of 'averaged' quantities, which become macroscopic in the limit $N \rightarrow \infty$. The important point is that symmetric sequences commute in this limit; more precisely

$$
\lim _{N \rightarrow \infty}\left\|A_{N} A_{N}^{\prime}-A_{N}^{\prime} A_{N}\right\|=0 .
$$

The averaging of 1-particle spin operators provides a clear example, and illustrates how $N \rightarrow \infty$ corresponds to $\hbar \rightarrow 0$. Thus let $A_{N}:=j_{N 1}(B)$ and $A_{N}^{\prime}:=j_{N 1}(C)$ with $B, C \in \mathcal{A}_{1}$. Then the fact that $\left[B_{(k)}, C_{(l)}\right]=0$ for $k \neq l$ implies that

$$
\left[\bar{B}^{(N)}, \bar{C}^{(N)}\right]=\frac{1}{N} \overline{[B, C]}^{(N)} \text {. }
$$

For example, if $\mathcal{A}_{1}=M_{2}(\mathbb{C})$ and if for $B$ and $C$ one takes the spin- $\frac{1}{2}$ operators $S_{j}=\frac{\hbar}{2} \sigma_{j}$ for $j=1,2,3$ ( $\sigma_{j}$ the Pauli matrices), then

$$
\left[\bar{S}_{j}^{(N)}, \bar{S}_{k}^{(N)}\right]=i \frac{\hbar}{N} \epsilon_{j k l} \bar{S}_{l}^{(N)}
$$

Thus we get commutation relations formally like those of the one-particle operators, except that Planck's constant $\hbar$ is replaced by $\hbar / N$.

We are now ready to define our continuous field of algebras. A section of the field with fibers 6.16 is a sequence $A=\left(A_{0}, A_{1}, A_{2}, \cdots\right)$, with $A_{0} \in \mathcal{A}_{0}^{(c)}$ and $A_{N} \in \mathcal{A}_{1}^{N}$. We say that a sequence $A$ defines a continuous section of the field iff:

- $\left(A_{1}, A_{2}, \cdots\right)$ is approximately symmetric; i.e. for any $\varepsilon>0$ there is an $N_{\varepsilon}$ and a symmetric sequence $A^{\prime}$ such that $\left\|A_{N}-A_{N}^{\prime}\right\|<\varepsilon$ for all $N \geq N_{\varepsilon}$;

- $A_{0}(\omega)=\lim _{N \rightarrow \infty} \omega^{N}\left(A_{N}\right)$, where $\omega \in \mathcal{S}\left(\mathcal{A}_{1}\right)$ and $\omega^{N} \in \mathcal{S}\left(\mathcal{A}_{1}^{N}\right)$ is the tensor product of $N$ copies of $\omega$, i.e.

$$
\omega^{N}\left(B_{1} \otimes \cdots \otimes B_{N}\right)=\omega\left(B_{1}\right) \cdots \omega\left(B_{N}\right)
$$

This choice of continuous sections defines a continuous field of $C^{*}$-algebras over $I=0 \cup 1 / \mathbb{N}$ with fibers 6.16. In fact it follows that

$$
\lim _{N \rightarrow \infty}\left\|A_{N}\right\|=\left\|A_{0}\right\| .
$$

To sum up: the main point is that, in accordance with 6.21, the macroscopic quantities are organized in the limit $N \rightarrow \infty$ in to a commutative $C^{*}$-algebra isomorphic to $C\left(\mathcal{S}\left(\mathcal{A}_{1}\right)\right)$. 


\subsubsection{The quantum infinite: quasi-local sequences}

I now treat the quantum $N \rightarrow \infty$ limit of $N$ copies of the algebra $\mathcal{A}_{1}$. The key idea will be that of a quasilocal sequence $\left(A_{1}, A_{2}, \cdots\right)$ of algebra elements, with $A_{N} \in \mathcal{A}_{1}^{N}$. In constructing the continuous field of $C^{*}$-algebras, this notion will play an analogous role to that played in Section 6.2.3 by symmetric sequences. The idea is that the tail of a quasilocal sequence becomes arbitrarily close (in norm) to an element $A_{M} \otimes 1 \otimes 1 \otimes \cdots$, so that the sequence "ignores, in the limit" all but a finite part of the system. The intuition behind this restriction is that human limitation means we can observe only a finite part of the system. In any case, the restriction allows us to make precise the heuristic idea of the algebra for an infinite quantum system, which we might write heuristically as $\mathcal{A}_{1}^{\infty}=$ say $M_{2}(\mathbb{C})^{\infty}$.

Formally, the infinite quantum system is the inductive limit $C^{*}$-algebra

$$
\overline{\cup_{N \in \mathbb{N}} \mathcal{A}_{1}^{N}}
$$

of the family of $C^{*}$-algebras $\left(\mathcal{A}_{1}^{N}\right)$. Eq. 6.26 consists of all equivalence classes $[A] \equiv$ $A_{0}$ of quasilocal sequences $A=\left(A_{1}, A_{2}, \cdots\right)$, under the equivalence relation $A \sim B$ iff $\lim _{N \rightarrow \infty}\left\|A_{N}-B_{N}\right\|=0$. As the notation suggests, each $\mathcal{A}_{1}^{N}$ is contained in $\overline{\cup_{N \in \mathbb{N}} \mathcal{A}_{1}^{N}}$ as a $C^{*}$-subalgebra by identifying $A_{N} \in \mathcal{A}_{1}^{N}$ with a quasilocal sequence that after the $N$ th term just tensors with the identity, viz. the sequence $A=\left(0, \cdots, 0, A_{N}, A_{N} \otimes 1, A_{N} \otimes 1 \otimes 1, \cdots\right)$, and forming its equivalence class $[A] \equiv A_{0}$ in $\overline{\cup_{N \in \mathbb{N}} \mathcal{A}_{1}^{N}}$.

In Detail: A sequence $A=\left(A_{1}, A_{2}, \cdots\right)\left(A_{N} \in \mathcal{A}_{1}^{N}\right)$ is local if for some fixed $M$ and all $N \geq M: A_{N}=A_{M} \otimes 1 \otimes \cdots \otimes 1$ (with $N-M$ copies of the unit $1 \in \mathcal{A}_{1}$ ). A sequence is quasilocal when for any $\varepsilon>0$ there is an $N_{\varepsilon}$ and a local sequence $A^{\prime}$ such that $\left\|A_{N}-A_{N}^{\prime}\right\|<\varepsilon$ for all $N \geq N_{\varepsilon}$.

We now define the inductive limit $C^{*}$-algebra

$$
\overline{\cup_{N \in \mathbb{N}} \mathcal{A}_{1}^{N}}
$$

of the family of $C^{*}$-algebras $\left(\mathcal{A}_{1}^{N}\right)$ with respect to the inclusion maps $\mathcal{A}_{1}^{N} \hookrightarrow \mathcal{A}_{1}^{N+1}$ given by $A_{N} \mapsto A_{N} \otimes 1$. As a set, 6.27 consists of all equivalence classes $[A] \equiv A_{0}$ of quasilocal sequences $A$ under the equivalence relation $A \sim B$ when $\lim _{N \rightarrow \infty}\left\|A_{N}-B_{N}\right\|=0$. The norm on $\overline{\cup_{N \in \mathbb{N}} \mathcal{A}_{1}^{N}}$ is

$$
\left\|A_{0}\right\|=\lim _{N \rightarrow \infty}\left\|A_{N}\right\|,
$$

and other $C^{*}$-algebraic structure is inherited from the quasilocal sequences in the obvious way (e.g., $A_{0}^{*}=\left[A^{*}\right]$ with $A^{*}=\left(A_{1}^{*}, A_{2}^{*}, \cdots\right)$, etc.). Thus each $\mathcal{A}_{1}^{N}$ is contained in $\overline{\cup_{N \in \mathbb{N}} \mathcal{A}_{1}^{N}}$ as a $C^{*}$-subalgebra by identifying $A_{N} \in \mathcal{A}_{1}^{N}$ with the local sequence $A=\left(0, \cdots, 0, A_{N}, A_{N} \otimes 1, A_{N} \otimes 1 \otimes 1, \cdots\right)$, and forming its equivalence class $A_{0}$ in $\cup_{N \in \mathbb{N}} \mathcal{A}_{1}^{N}$.

So we define a second continuous field of $C^{*}$-algebras $\mathcal{A}^{(q)}$ over $0 \cup 1 / \mathbb{N}$, with fibers

$$
\begin{aligned}
\mathcal{A}_{0}^{(q)} & =\overline{\cup_{N \in \mathbb{N}} \mathcal{A}_{1}^{N}} ; \\
\mathcal{A}_{1 / N}^{(q)} & =\mathcal{A}_{1}^{N} .
\end{aligned}
$$


by declaring that the continuous sections are of the form $\left(A_{0}, A_{1}, A_{2}, \cdots\right)$ where $\left(A_{1}, A_{2}, \cdots\right)$ is quasilocal and $A_{0}$ is defined to be the equivalence class of this quasilocal sequence, as just explained.

For $N<\infty$ this field has the same fibers

$$
\mathcal{A}_{1 / N}^{(q)}=\mathcal{A}_{1 / N}^{(c)}=\mathcal{A}_{1}^{N}
$$

as Section 6.2.3 s continuous field $\mathcal{A}^{(c)}$, but the fiber $\mathcal{A}_{0}^{(q)}$ is completely different from $\mathcal{A}_{0}^{(c)}$. For if $\mathcal{A}_{1}$ is noncommutative then so is $\mathcal{A}_{0}^{(q)}$, since it contains all $\mathcal{A}_{1}^{N}$.

\subsubsection{Comparing the classical and quantum limits: classical states and the de Finetti theorem}

One natural way to study the relations between the fields $\mathcal{A}^{(c)}$ and $\mathcal{A}^{(q)}$ is to consider those families of abstract states $\left(\omega_{1}, \omega_{1 / 2}, \cdots, \omega_{1 / N}, \cdots\right)\left(\omega_{1 / N}\right.$ is a state on $\left.\mathcal{A}_{1}^{N}\right)$ that have appropriate limit states on both $\mathcal{A}^{(c)}$ and $\mathcal{A}^{(q)}$. (Here 'appropriate' is made precise in terms of Section 6.2.2 s notion of a continuous field of states.) In this Section, I introduce the most important such family, the permutation-invariant states. We will see in Section 6.3.1 how they yield superselection sectors, especially in Section 6.2.1 s prototype system, spin chains.

Of course, any state $\omega_{0}^{(q)}$ on $\mathcal{A}_{0}^{(q)}$ defines a state $\omega_{0 \mid 1 / N}^{(q)}$ on each $\mathcal{A}_{1}^{N}$ by restriction; and the sequence of these states have the given $\omega_{0}^{(q)}$ as their appropriate limit state on $\mathcal{A}_{0}^{(q)}$. If this sequence of states also converges with respect to the other continuous field of algebras $\mathcal{A}^{(c)}$, i.e. converges to some state $\omega_{0}^{(c)}$ on $\mathcal{A}_{0}^{(c)}$, then the given state $\omega_{0}^{(q)}$ is called classical.

We specialize to an important class of classical states, viz. those that are "indifferent to" the label $N$ of the component systems (e.g. of the sites in the spin chain). Formally, we say that a state $\omega_{0}^{(q)}$ on $\mathcal{A}_{0}^{(q)}$ is permutation-invariant if its restrictions to each of the $\mathcal{A}_{1}^{N}$ is invariant under the natural action of the symmetric group $S_{N}$ on $\mathcal{A}_{1}^{N}$.

For our purposes, the important point about these states is that they give a close quantum analogue of the de Finetti representation theorem. Roughly speaking, this theorem says that any classical probability measure on an infinite Cartesian power probability space $X^{\infty}:=X \times X \times \cdots$ that is permutation-invariant (under permutations between copies of the factor space $X$ ) is a unique mixture of (i.e. has a unique integral decomposition in terms of) infinite product probability measures $p^{\infty}$ given by $p^{\infty}\left(Y_{1} \times Y_{2} \times \cdots\right):=p\left(Y_{1}\right) \cdot p\left(Y_{2}\right) \ldots$ (with $Y_{i}$ in the sigma-algebra of the $i$ th copy of $X$ ) for some probability measure $p$ on $X$. Section 6.3.1 will describe how the quantum analogue of this theorem gives a precise yet general framework for the emergence of superselection.

In Detail: Consider those families of states $\left(\omega_{1}, \omega_{1 / 2}, \cdots, \omega_{1 / N}, \cdots\right)$ (where $\omega_{1 / N}$ is a state on $\mathcal{A}_{1}^{N}$ ) that have limit states both $\omega_{0}^{(c)}$ on $\mathcal{A}_{0}^{(c)}$ and and $\omega_{0}^{(q)}$ on $\mathcal{A}_{0}^{(q)}$, such that the ensuing families $\left(\omega_{0}^{(c)}, \omega_{1}, \omega_{1 / 2}, \cdots\right)$ and $\left(\omega_{0}^{(q)}, \omega_{1}, \omega_{1 / 2}, \cdots\right)$ are continuous fields of states on $\mathcal{A}^{(c)}$ and on $\mathcal{A}^{(q)}$ (in the sense of Section 6.2.2).

Any state $\omega_{0}^{(q)}$ on $\mathcal{A}_{0}^{(q)}$ defines a state $\omega_{0 \mid 1 / N}^{(q)}$ on $\mathcal{A}_{1}^{N}$ by restriction, and the en- 
suing field of states on $\mathcal{A}^{(q)}$ is clearly continuous. (Conversely, any continuous field $\left(\omega_{0}^{(q)}, \omega_{1}, \omega_{1 / 2}, \ldots, \omega_{1 / N}, \ldots\right)$ of states on $\mathcal{A}^{(q)}$ becomes arbitrarily close to a field of the above type for $N$ large.) But the restrictions $\omega_{0 \mid 1 / N}^{(q)}$ of a given state $\omega_{0}^{(q)}$ on $\mathcal{A}_{0}^{(q)}$ to $\mathcal{A}_{1}^{N}$ may well not converge to a state $\omega_{0}^{(c)}$ on $\mathcal{A}_{0}^{(c)}$ for $N \rightarrow \infty$. States $\omega_{0}^{(q)}$ on $\overline{\cup_{N \in \mathbb{N}} \mathcal{A}_{1}^{N}}$ whose restrictions $\omega_{0 \mid 1 / N}^{(q)}$ do converge to a state $\omega_{0}^{(c)}$ on $\mathcal{A}_{0}^{(c)}$ are called classical.

In other words (cf. the definition of $\mathcal{A}_{0}^{(c)}$ especially eq. 6.24): $\omega_{0}^{(q)}$ is classical when there exists a probability measure $\mu_{0}$ on $\mathcal{S}\left(\mathcal{A}_{1}\right)$ such that

$$
\lim _{N \rightarrow \infty} \int_{\mathcal{S}\left(\mathcal{A}_{1}\right)} d \mu_{0}(\rho)\left(\rho^{N}\left(A_{N}\right)-\omega_{0 \mid 1 / N}^{(q)}\left(A_{N}\right)\right)=0
$$

for each approximately symmetric sequence $\left(A_{1}, A_{2}, \ldots\right)$. In other words: a classical state $\omega_{0}^{(q)}$ with limit state $\omega_{0}^{(c)}$ on $C\left(\mathcal{S}\left(\mathcal{A}_{1}\right)\right)$ defines a probability measure $\mu_{0}$ on $\mathcal{S}\left(\mathcal{A}_{1}\right)$ by

$$
\omega_{0}^{(c)}(f)=\int_{\mathcal{S}\left(\mathcal{A}_{1}\right)} d \mu_{0} f
$$

which describes the probability distribution of the macroscopic quantities in that state.

We now make this more concrete by specializing to an important class of classical states. We say that a state $\omega$ on $\overline{\cup_{N \in \mathbb{N}} \mathcal{A}_{1}^{N}}$ is permutation-invariant when each of its restrictions to $\mathcal{A}_{1}^{N}$ is invariant under the natural action of $\mathfrak{S}_{N}$ on $\mathcal{A}_{1}^{N}$ (i.e. $\sigma \in \mathfrak{S}_{N}$ maps an elementary tensor $A_{N}=B_{1} \otimes \cdots \otimes B_{N} \in \mathcal{A}_{1}^{N}$ to $B_{\sigma(1)} \otimes \cdots \otimes B_{\sigma(N)}$, cf. 6.17).

We can now state the quantum analogue of the de Finetti representation theorem: a permutation-invariant state on $\mathcal{A}_{0}^{(q)}=\overline{\cup_{N \in \mathbb{N}} \mathcal{A}_{1}^{N}}$ is a unique mixture of (i.e. has a unique integral decomposition in terms of) infinite product states $\rho^{\infty}$, that are defined (with $\left.\rho \in \mathcal{S}\left(\mathcal{A}_{1}\right)\right)$ by saying that if $A_{0} \in \mathcal{A}_{0}^{(q)}$ is an equivalence class $\left[A_{1}, A_{2}, \cdots\right]$, then (cf. 6.24)

$$
\rho^{\infty}\left(A_{0}\right)=\lim _{N \rightarrow \infty} \rho^{N}\left(A_{N}\right) .
$$

An equivalent definition is to say that the restriction of $\rho^{\infty}$ to any $\mathcal{A}_{1}^{N} \subset \mathcal{A}_{0}^{(q)}$ is given by $\otimes^{N} \rho$.

In other words, the theorem says: any permutation-invariant state $\omega_{0}^{(q)}$ has a unique decomposition

$$
\omega_{0}^{(q)}\left(A_{0}\right)=\int_{\mathcal{S}\left(\mathcal{A}_{1}\right)} d \mu(\rho) \rho^{\infty}\left(A_{0}\right)
$$

where $\mu$ is a probability measure on $\mathcal{S}\left(\mathcal{A}_{1}\right)$ and $A_{0} \in \mathcal{A}_{0}^{(q)}$. We can also state this in more geometric language, as follows. The set $\mathcal{S}^{\mathfrak{S}}$ of all permutation-invariant states in $\mathcal{S}\left(\mathcal{A}_{0}^{(q)}\right)$ is a compact convex set, and is the (weak*-closed) convex hull of its (extreme) boundary $\partial_{e} \mathcal{S}^{\mathfrak{S}}$. So the claim of the theorem is that this boundary consists of the infinite product states (and so is isomorphic to $\mathcal{S}\left(\mathcal{A}_{1}\right)$ in the obvious way).

To sum up this Section, especially 6.31, 6.32 and 6.34 - If $\omega_{0}^{(q)}$ is permutationinvariant, then it is classical. The associated limit state $\omega_{0}^{(c)}$ on $\mathcal{A}_{0}^{(c)}$ is characterized by the fact that the measure $\mu_{0}$ in 6.32 coincides with the measure $\mu$ in 6.34 . 


\subsection{The claims illustrated by emergent superselection}

The material in Section 6.2 gives a precise and powerful framework for understanding the emergence of superselection in the $N \rightarrow \infty$ limit. It will be clearest to first summarize this (Section 6.3.1, again following Landsman (2006)), and then spell out the illustrations of my claims (Section 6.3.2 et seq.).

\subsubsection{Superselection from permutation-invariant states, in spin chains}

I begin with generalities, and then return to spin-chains. In the algebraic approach to quantum theory, a superselection sector is taken to be an appropriate equivalence class (under unitary isomorphism) of irreducible representations of the system's abstract the algebra of quantities $\mathcal{A}$. The word 'appropriate' reflects the fact that most irreducible representations of a typical $C^{*}$-algebra $\mathcal{A}$ used in physics are physically irrelevant, and so need to be excluded; (jargon: one needs a selection criterion). Here, we take the algebra of quantities to be $\mathcal{A}_{0}^{(q)}$; and take an (equivalence class of) irreducible representations of $\mathcal{A}_{0}^{(q)}$ to be a superselection sector iff it corresponds to a permutation-invariant pure state on $\mathcal{A}_{0}^{(q)}$. (Here 'correspond' is made precise using the GNS theorem, viz. as equivalence to the GNS-representation of the permutation-invariant state.) With this selection criterion, the results in Section 6.2, especially at 6.33 onwards, trivially imply that there is a bijective correspondence between pure states on $\mathcal{A}_{1}$ and superselection sectors of $\mathcal{A}_{0}^{(q)}$.

The results are vividly illustrated by spin chains. In Section 6.2.1, I did not give details about how to build the infinite tensor product state space $\mathcal{H}_{1}^{\infty}$, with say $\mathcal{H}_{1}=\mathbb{C}^{2}$. But as one would hope, we have: If $\left(e_{i}\right)$ is some basis of $\mathbb{C}^{2}$, an orthonormal basis of $\mathcal{H}_{1}^{\infty}$ consists of all different infinite strings $e_{i_{1}} \otimes \cdots e_{i_{n}} \otimes \cdots$, where $e_{i_{n}}$ is $e_{i}$ regarded as a vector in $\mathbb{C}^{2}$. (And similarly, when we choose the "building-block" algebra $\mathcal{A}_{1} \neq \mathbb{C}^{2}$.) We denote the multi-index $\left(i_{1}, \ldots, i_{n}, \ldots\right)$ simply by $I$, and the corresponding basis vector by $e_{I}$. This Hilbert space $\mathcal{H}_{1}^{\infty}$ carries a natural faithful representation $\pi$ of $\mathcal{A}_{0}^{(q)}$ : if $A_{0} \in \mathcal{A}_{0}^{(q)}$ is an equivalence class $\left[A_{1}, A_{2}, \cdots\right]$, then $\pi\left(A_{0}\right) e_{I}=\lim _{N \rightarrow \infty} A_{N} e_{i}$, where $A_{N}$ acts on the first $N$ components of $e_{I}$ and leaves the remainder unchanged.

Now the important point is that although each $\mathcal{A}_{1}^{N}$ acts irreducibly on $\mathcal{H}_{1}^{N}$, the representation $\pi\left(\mathcal{A}_{0}^{(q)}\right)$ on $\mathcal{H}_{1}^{\infty}$ thus constructed is highly reducible. The reason for this is that by definition (quasi-) local elements of $\mathcal{A}_{0}^{(q)}$ leave the infinite tail of a vector in $\mathcal{H}_{1}^{\infty}$ (almost) unaffected, so that vectors with different tails lie in different superselection sectors. Without the quasi-locality condition on the elements of $\mathcal{A}_{0}^{(q)}$, no superselection rules would arise.

For example, in terms of the usual basis $\left\{\uparrow=\left(\begin{array}{l}1 \\ 0\end{array}\right), \downarrow=\left(\begin{array}{l}0 \\ 1\end{array}\right)\right\}$ of $\mathbb{C}^{2}$, the vectors $\Psi_{\uparrow}=\uparrow \otimes \uparrow \cdots \uparrow \cdots$ (i.e. an infinite product of 'up' vectors) and $\Psi_{\downarrow}=\downarrow \otimes \downarrow \cdots \downarrow \cdots$ (i.e. an infinite product of 'down' vectors) lie in different sectors. (Cf. items (a) and (b) in Section 6.2.1, and (iv) in Section 6.2.3.) The reason why the inner product $\left(\Psi_{\uparrow}, \pi(A) \Psi_{\downarrow}\right)$ vanishes for any $A \in \mathcal{A}_{0}^{(q)}$ is that for local quantities $A$ one has $\pi(A)=A_{M} \otimes 1 \otimes \cdots 1 \cdots$ for some $A_{M} \in \mathcal{B}\left(\mathcal{H}_{M}\right)$; the inner product in question therefore involves infinitely many 
factors $(\uparrow, 1 \downarrow)=(\uparrow, \downarrow)=0$. For quasilocal $A$ the operator $\pi(A)$ might have a small nontrivial tail, but again the inner product vanishes by an approximation argument.

More generally, elementary analysis shows that $\left(\Psi_{u}, \pi(A) \Psi_{v}\right)=0$ whenever $\Psi_{u}=\otimes^{\infty} u$ and $\Psi_{v}=\otimes^{\infty} v$ for unit vectors $u, v \in \mathbb{C}^{2}$ with $u \neq v$. The corresponding vector states $\psi_{u}$ and $\psi_{v}$ on $\mathcal{A}_{0}^{(q)}$ (i.e. $\psi_{u}(A)=\left(\Psi_{u}, \pi(A) \Psi_{u}\right)$ etc.) are obviously permutation-invariant and hence classical. Identifying $\mathcal{S}\left(M_{2}(\mathbb{C})\right)$ with $B^{3} \subset \mathbb{R}^{3}$, the corresponding limit state $\left(\psi_{u}\right)_{0}$ on $\mathcal{A}_{0}^{(c)}$ defined by $\psi_{u}$ is given by (evaluation at) the point $\tilde{u}=(x, y, z)$ of $\partial B^{3}=S^{2}$ (i.e. the two-sphere) for which the corresponding density matrix $\rho(\tilde{u})$ is the projection operator onto $u$. (It follows that $\psi_{u}$ and $\psi_{v}$ are unitarily inequivalent.)

We conclude that each unit vector $u \in \mathbb{C}^{2}$ determines a superselection sector $\pi_{u}$, namely the GNS-representation of the corresponding state $\psi_{u}$, and that each such sector is realized as a subspace $\mathcal{H}_{u}$ of $\mathcal{H}_{1}^{\infty}$ (viz. $\mathcal{H}_{u}=\overline{\pi\left(\mathcal{A}_{0}^{(q)}\right) \Psi_{u}}$ ). Moreover, since a permutation-invariant state on $\mathcal{A}_{0}^{(q)}$ is pure iff it is of the form $\psi_{u}$, these are all the superselection sectors. Thus we have the subspace (of $\mathcal{H}_{1}^{\infty}$ ) and subrepresentation (of $\pi$ )

$$
\begin{aligned}
\mathcal{H}_{\mathfrak{S}} & :=\oplus_{\tilde{u} \in S^{2}} \mathcal{H}_{u} \\
\pi_{\mathfrak{S}}\left(\mathcal{A}_{0}^{(q)}\right) & :=\oplus_{\tilde{u} \in S^{2}} \pi_{u}\left(\mathcal{A}_{0}^{(q)}\right),
\end{aligned}
$$

where $\pi_{u}$ is simply the restriction of $\pi$ to $\mathcal{H}_{u} \subset \mathcal{H}_{1}^{\infty}$.

In the presence of superselection, there are operators that distinguish different sectors whilst being a multiple of the unit in each sector; cf. items (c) and (d) of Section 6.2.1. In the framework developed from Section 6.2.2 onwards, these operators are the macroscopic quantities of Section 6.2.3. In fact, one can show for any approximately symmetric sequence $\left(A_{1}, A_{2}, \cdots\right)$ the limit

$$
\bar{A}=\lim _{N \rightarrow \infty} \pi_{\mathfrak{S}}\left(A_{N}\right)
$$

exists in the strong operator topology on $\mathcal{B}\left(\mathcal{H}_{\mathfrak{S}}\right)$. Moreover, let $A_{0} \in \mathcal{A}_{0}^{(c)}=C\left(\mathcal{S}\left(\mathcal{A}_{1}\right)\right)$ be the function defined by the given sequence (recall that $A_{0}(\omega)=\lim _{N \rightarrow \infty} \omega^{N}\left(A_{N}\right)$, cf. eq. 6.24). Then the map $A_{0} \mapsto \bar{A}$ defines a faithful representation of $\mathcal{A}_{0}^{(c)}$ on $\mathcal{H}_{\mathfrak{S}}$, which we again call $\pi_{\mathfrak{S}}$. A calculation shows that $\pi_{\mathfrak{S}}\left(A_{0}\right) \Psi=A_{0}(\tilde{u}) \Psi$ for $\Psi \in \mathcal{H}_{u}$, or, in other words,

$$
\pi_{\mathfrak{S}}\left(A_{0}\right)=\oplus_{\tilde{u} \in S^{2}} A_{0}(\tilde{u}) 1_{\mathcal{H}_{u}} .
$$

Thus the $\pi_{\mathfrak{S}}\left(A_{0}\right)$ are indeed the promised operators.

\subsubsection{Emergence in the limit: with reduction - and without}

I turn at last to how the example of superselection illustrates my claims. It is clear from the wealth of details in the preceding Sections that my positive claims, (1:Deduce) and (2:Before), can be richly illustrated, in terms of both quantities and states. So I will confine myself to mentioning the obvious illustrations, and referring to the previous discussions and equations. 
As to (1:Deduce): we again have 'reduction as deduction' in as strong a sense as you could demand-provided we take the limit. There are several illustrations, some concerning quantities or algebras of them, some concerning states. I begin with two brief cases, referring back to equations in Subsections of Section 6.2.

An obvious case, which concerns quantities, is that symmetric sequences $\left(A_{N}\right)$ of quantities, $A_{N} \in \mathcal{A}_{1}^{N}$, commute in the limit $N \rightarrow \infty$. Recall eq. 6.21 in Section 6.2.3, Another obvious case, which equally concerns states, is spin density in spin chains, as in part (c) of Section 6.2.1. Thus recall the limiting behaviour of the average, over $2 N+1$ sites, of the spin matrices, i.e. the limiting behaviour of $\mathbf{m}_{N}^{(+)}$defined by eq. 6.5. The discussion from eq. 6.6 to eq. 6.9 deduced that the limiting spin density has the same value, $\mathbf{k}$, for all states in the representation $\mathcal{H}^{(+)}$; and so it is a classical quantity, or superselection operator.

Here are three more substantial cases of such a deduction, using my mnemonic notations, $T_{b}$ and $T_{t}$ : that is, cases where $T_{b}$ implies $T_{t}$. The first two concern quantities, the third concerns states. Here, I shall mostly refer back to the summary in Section 6.3.1.

(A): Take as $T_{b}$ the continuous field of $C^{*}$-algebras $\mathcal{A}^{(q)}$, together with its representation theory: or more modestly, together with that part of its representation theory that deals with permutation-invariant states. Take as $T_{t}$ a statement of superselection, e.g. that $\mathcal{A}_{0}^{(q)}$ acts (highly!) reducibly on the infinite-system state space $\mathcal{H}_{1}^{\infty}$. Then we have just seen in Section 6.3.1 that $T_{b}$ implies $T_{t}$. For the requirement for sequences of operators to be quasi-local makes the inner products between states in different sectors of $\mathcal{H}_{1}^{\infty}$ vanish. Compare the discussion leading up to eq. 6.35,

(B): Let $T_{b}$ be the continuous field of $C^{*}$-algebras $\mathcal{A}^{(c)}$, together with $\pi_{\mathfrak{S}}$, the faithful representation of $\mathcal{A}_{0}^{(c)}$ on $\mathcal{H}_{\mathfrak{S}}$ defined by eq. 6.36. Take $T_{t}$ to state that there are superselection operators (classical quantities) that restrict to the identity on each sector $\mathcal{H}_{\mathfrak{S}}$. Then we have just seen at the end of Section 6.3.1 that $T_{b}$ implies $T_{t}$. For compare the discussion leading up to eq. 6.37; any approximately symmetric sequence $\left(A_{N}\right)$ (and so any continuous section of the field) defines such a classical quantity, viz. $\pi_{\mathfrak{S}}\left(A_{0}\right)$.

(C): We can focus instead on states. Take $T_{b}$ to encompass both continuous fields of $C^{*}$-algebras, $\mathcal{A}^{(q)}$ and $\mathcal{A}^{(c)}$, and to consider families of states on them. Take $T_{t}$ to be the theory of classical states, especially permutation-invariant, states $\omega_{0}^{(q)}$; or more modestly, to be just the quantum de Finetti representation theorem, eq. 6.34. Then Section 6.2.5 shows that $T_{b}$ implies $T_{t}$.

I turn to the "the other side of the coin" of (1:Deduce): the failure of the reduction at finite $N$. For (A) and (B), the point is familiar from elementary quantum theory. Finite $N$ means there is no algebra $\mathcal{A}_{0}^{(c)}$ or $\mathcal{A}_{0}^{(q)}$ to consider: there are only the algebras $\mathcal{A}_{N}^{(c)} \equiv \mathcal{A}_{N}^{(q)}:=\mathcal{A}_{1}^{N}$. But in general, if $\mathcal{A}_{1}$ acts irreducibly on $\mathcal{H}_{1}$, then so does $\mathcal{A}_{1}^{N}$ on $\mathcal{H}_{1}^{N}$. So (A) fails. And then Schur's Lemma - that if an algebra acts irreducibly, its commutant is trivial -immmediately implies that (B) fails, i.e. there are only trivial superselection operators.

As to (C), the point is less familiar. But it is again straightforward, especially if we limit ourselves by choosing $T_{t}$ to be the quantum de Finetti representation theorem, 
not the whole theory of classical states; (of course choosing a weaker $T_{t}$ adds to the dialectical force of the point that it is not implied). Here the point is that for finite $N$, the theorem fails. The reason is essentially the same as for the corresponding failure of the classical theorem for finite $N$. In short, finite $N$ corresponds to drawing from an urn without replacement, rather than with replacement. In the classical case, this means that a permutation-invariant probability measure is a unique mixture, not of product measures, but of hypergeometric measures (cf. Diaconis 1977, Diaconis and Freedman 1980, Jeffrey 1988, pp. 240-245). Similar remarks apply in the quantum case: (C) of Section 6.3.3 will give a few more details.

Finally, I note two respects in which this example's illustration of (1:Deduce) is similar to that given by fractals, and dissimilar to that given by the method of arbitrary functions. I noted these respects at the end of Section 5.2.1. First: the emergent behaviour involves mathematical structures which are new at the limit (such as unitarily inequivalent representations, and so superselection). This is like fractals, where the emergent behaviour involved non-integer dimension at the limit; but unlike the method of arbitrary functions, where the emergent behaviour involved, more simply, deducing the limiting behaviour of functions given at finite $N$.

The second respect is related to the first. Namely, this example considers an infinite quantum system: in Section 3.1's notation, an infinite system $\sigma(\infty)$. Again, this is like fractals, where the Cantor set, Koch snowflake etc. count as infinite systems; but unlike the method of arbitrary functions, since, for example, no roulette wheel has infinitely many arcs.

\subsubsection{Emergence before the limit}

(2:Before) claims that before the limit, there is emergence in a weaker but still vivid sense. This claim is illustrated in a manner parallel to my previous examples: the method of arbitrary functions, and fractals. In short, one just has to interpret (sensibly!) the example's formalism describing finite $N$. And as in those examples, the discussion can be made vivid by referring to practical purposes. There, I cited the practical purposes of a casino in making a wheel that is fair enough; and the purposes of a film studio in making an image look fractal at small enough spatial scales. Similarly here: imagine an experimental physicist making, or a theoretical physicist describing, a sample or device comprising $N$ atoms or sites (perhaps for a nanotechnology project), with $N$ large enough for behaviour characteristic of superselection to occur.

Again, one can pick several illustrations. I shall give three. The first concerns states in spin chains as in Section 6.2.1, the second concerns symmetrized quantities as in Section 6.2.3, the third returns us to the quantum de Finetti representation theorem, as in Section 6.3.2. All three have the merit (absent from my discussion of my previous examples!) of being quantitative about the "rate of emergence": about how large an $N$ is needed for emergent behaviour.

(A): The discussion in part (c) of Section 6.2.1 concentrated on the limiting behaviour of the expectation value of $\mathbf{m}_{N}^{(+)}$on states in $\mathcal{H}^{(+)}$. We deduced (cf. eq. 6.6 to eq. 6.9) 
that the limiting value is $\mathbf{k}$, the unit vector in the $z$-direction. But that discussion can of course be adapted to entail statements about the situation for finite $N$. To give one example: consider the $x$ or $y$ component of the matrix element of $\mathbf{m}_{N}^{(+)}$between two elements $\phi_{s}^{(+)}, \phi_{s^{\prime}}^{(+)}$of the basis of $\mathcal{H}^{(+)}$that correspond to two doubly infinite $(+1,-1)$ sequences, $s=\left(s_{n}\right)_{n \in Z}$ and $s^{\prime}=\left(s_{n}^{\prime}\right)_{n \in Z}$, both with all but finitely many elements $\left(s_{n}\right.$ or $s_{n}^{\prime}$ ) equal to +1 . In part (c) of Section 6.2.1, we argued that these matrix elements tend to 0 as $N \rightarrow \infty$. But as to finite $N$ : if we know that $s_{n}=s_{n}^{\prime}$ for all $n$ with $|n|>M$, we can readily calculate how large $N$ needs to be for the $2 N+1$ denominator to make the $x$ or $y$ component of the matrix element have modulus less than any given $\varepsilon$.

(B): The discussion surrounding eq. 6.22 and 6.23 in Section 6.2.1 concentrated on the limiting behaviour, as $N \rightarrow \infty$, of the commutator of averages over $N$ particles of 1-particle spin operators. But as in (A), that discussion of course entails statements about the situation for finite $N$. Indeed, eq. 6.22 and 6.23 say explicitly that these commutators are proportional to $1 / N$.

(C): Section 6.3 .2 reported that the quantum de Finetti representation theorem, eq. 6.34, failed for finite $N$ : a permutation-invariant state on $N$ quantum systems need not be mixture of product states. But in recent years, various finite- $N$ analogues have been proven, with the following flavour: any state of $M$ systems, with $M<N$, that is obtained from a permutation-invariant state on $N$ systems, by tracing out (partial tracing over) $N-M$ systems, can be approximated by a mixture of product states on the $M$ systems (i.e. states $\left.\omega^{\otimes M}\right)$, with an error that goes to zero with the ratio $M / N$, e.g. an error like $O(M / N)$; (Koenig and Renner 2005, Renner 2007, Koenig and Mitchison 2007).

Note finally that as with my previous two examples, in these three illustrations of (2:Before) we again see the Straightforward Justification of Section 3.3.3 in action. I will not labour the point: I will just quote Landsman's own statement of the idea of that Section's fifth paragraph, as applied to the averaging process used (in Section 6.2.3) in defining macroscopic quantities. Thus Landsman writes: 'the limit $N \rightarrow \infty$ is valid whenever averaging over $N=10^{23}$ particles is well approximated by averaging over an arbitrarily larger number $N$ (which, then, one might as well let go to infinity)' (Landsman 2006, preamble to Section 6; p. 493).

\subsubsection{Supervenience is a red herring}

As in two previous examples, I shall be brief about my third claim, (3:Herring): that although various supervenience theses are true, they give little or no insight into the emergent behaviour, or more generally into "what is going on" in the example. The reason is as in the previous examples: there is no connection between supervenience's idea of a variety of ways to have a higher-level property $P$ (in particular the example's emergent property) and the limit processes on which the example turns.

But to save space, I will not formulate any such supervenience theses. I leave it as an exercise (!) to formulate how a system's having an emergent property, such as a specific value for a specific classical quantity, or a superselection operator, supervenes on the system's microstate, i.e. on the sequence of states assigned to the algebras $\mathcal{A}_{1}^{N}$ 
for successively larger $N$-particle (or site) sub-systems. Suffice it to say here that the important points will be that:

(i) the value (indeed, even just the well-definedness) of the quantity or operator, in the strong sense of (1:Deduce) but not the weak sense of (2:Before), needs one to take the limit $N \rightarrow \infty$, in the classical (Section 6.2.3) and-or quantum (Section 6.2.4) version; cf. the end of Section 6.3.1;

(ii) the idea of supervenience on the microstate makes no connection with taking this limit.

\subsection{Summing up superselection}

To emphasise the parallel between this long example and the previous two, let me sum up with a list of six morals which are parallel to those in Section 5.4. As in that Section, it would be flogging a dead horse to again make explicit my four claims, or Section 3.3.3 s Straightforward Justification, or the parallels with previous examples.

(i): The large finite is often well-modelled by the infinite.

(ii): Such models are often justified in a straightforward, even obvious, way, by mathematical convenience and empirical success.

(iii): The infinite often brings new mathematical structure: in this example, superselection, and associated notions like unitarily inequivalent representations.

(iv): Nevertheless, there is often a reduction: the emergent superselection properties, and the associated behaviour, are reducible to a sufficiently rich theory that takes the infinite limit.

(v): On the other hand, one can often see emergent behaviour on the way to the limit. Indeed, the larger your error bar, e.g. for detecting experimental statistics characteristic of some commuting operators, the lower the number of particles (or lattice sites) $N$ for which your experiments will confirm (more precisely: you think your experiments confirm!) the properties and behaviour that are characteristic of superselection.

(vi): Various supervenience theses hold-but they are trivial, or at least scientifically useless.

\section{$7 \quad$ Phase transitions}

Lack of space means I must deal much more briefly with my fourth example. This is unfortunate, for two reasons. Scientifically, this example represents a much larger and more controversial topic than my previous ones. And philosophically, it has been a prominent example in recent controversy about whether the $N=\infty$ limit is "physical real", and whether a "singular" limit is necessary for emergence (e.g. Callender (2001, Section 5, pp. 547-551), Liu (2001, Sections 2-3, pp. S326-S341), Batterman (2005, Section 4, pp. 233-237); and more recently, Mainwood (2006, Chapters 3,4; 2006a), Bangu (2009, Section 5, pp. 496-502), Menon and Callender (2011, especially Sections 3,4)). But sufficient unto the day is the work thereof! I have already declared my general position in these 
controversies (especially in Sections 1 and 3). So in this Section, it will be enough to sketch: (i) how statistical mechanics treats phase transitions by taking a limit, in which the number of constituent particles (or sites in a lattice) $N$ goes to infinity (Section 7.1); and (ii) how this treatment illustrates my claims (Section 7.2).

For more information, I especially recommend: (i) accounts by masters of the subject, such as Emch and Liu (2002, Chapters 11-14) and Kadanoff (2009, 2010, 2010a), which treat the technicalities and history, as well as the conceptual foundations, of the subject; and (ii) Mainwood (2006, Chapters 3,4; 2006a), to which I am much indebted, especially in Section 7.2.1s treatment of phase transitions in finite $N$ systems, and Section [7.2.2 $\mathrm{s}$ discussion of cross-over.

\subsection{Phase transitions and thermodynamics}

\subsubsection{Separating issues and limiting scope}

This example is an aspect of a very large topic, the "emergence" of thermodynamics from statistical mechanics - around which debates about the reducibility of one to the other continue. This topic is very large, for various reasons. Three obvious ones are: (i) both thermodynamics and statistical mechanics are entire sciences; (ii) statistical mechanics, and so this topic, can be developed in either classical or quantum terms; (iii) there is no single agreed formalism for statistical mechanics (unlike e.g. quantum mechanics).

Phase transitions are themselves a large topic: there are several classification schemes for them, and various approaches to understanding them - some of which come in both classical and quantum versions. I will specialize to just one aspect; which will however be enough to illustrate my claims. Namely: the fact (on most approaches!) that for statistical mechanical systems, getting a (theoretical description of) a phase transition requires that one take a limit (often called 'the thermodynamic limit'), in which the number of constituent particles (or sites in a lattice) $N$ goes to infinity. In brief, this means something like: both the number $N$ of constituent particles (or sites), and the volume $V$ of the system tend to infinity, while the density $\rho=N / V$ remains fixed. More details in Section 7.1.2.

Even for this one aspect, I will have to restrict myself severely. Three main limitations are:

1): As regards philosophy, I impose a self-denying ordinance about the controversies mentioned in this Section's preamble: apart from what I have already said in Sections 1 and 3 - and one remark in Section 7.2.1.

2): In physics, how to understand phase transitions is an ongoing research area. For our purposes, the main limiting (i.e. embarrassing!) fact is that most systems do not have a well-defined thermodynamic limit - so that all that follows is of limited scope.

3): Various detailed justifications can be given for phase transitions requiring us to take the thermodynamic limit; and Section 7.1 .2 will only sketch a general argument, and mention two examples. Much needs to be (and has been!) said by way of assessing these

justifications - but I will not enter into this here. But by way of emphasizing how open all 
these issues are, I note that some physicists have developed frameworks for understanding phase transitions without taking the thermodynamic limit (Gross (2001)).

\subsubsection{The thermodynamic limit}

I will first give a broad description of the need for the thermodynamic limit; and then a classical and a quantum example to show how the limit secures new mathematical structure appropriate for describing phase transitions; (Section 7.1.2, A). Then in Section 7.1.2.B, I will give a classical example of approaching the limit. This example will be the phase transition of a ferromagnet at sub-critical temperatures. It has the merit of being very simple, and of developing Section 3.3.2 s dissolution of the "mystery" of describing a finite- $N$ system with a model using infinite $N$.

7.1.2. A: The need for the limit:- For classical physics, the brutal summary of why we need this limit is as follows. Statistical mechanics follows thermodynamics in representing phase transitions by non-analyticities of the free energy $F$. But a non-analyticity cannot occur for the free energy of a system with finitely many constituent particles (or analogously: lattice sites). So statistical mechanics considers a system with infinitely many

particles or sites, $N=\infty$. One gets some control over this idea by subjecting the limiting process, $N \rightarrow \infty$, to physically-motivated conditions like keeping the density constant, i.e. letting the volume $V$ of the system also go to infinity, while $N / V$ is constant. As we would expect - especially given my previous examples! - this infinite limit gives new mathematical structures: which happily turn out to describe phase transitions - in many cases, in remarkable quantitative detail.

But to make my ferromagnet example comprehensible, I need to spell out this line of argument in a bit more detail. In Gibbsian statistical mechanics, we postulate that the probability of a state $s$ is proportional to $\exp (-H(s) / k T) \equiv \exp (-\beta H(s))$, where $\beta:=\frac{1}{k T}$ is the inverse temperature and $k$ is Boltzmann's constant. That is:

$$
\operatorname{prob}(s)=\exp (-\beta H(s)) / Z
$$

where the normalization factor $Z$, the partition function, is the sum (or integral) over all states, and defines the free energy $F$ as:

$$
Z=\Sigma_{s} \exp (-\beta H(s))=: \exp (-\beta F)
$$

Thus $F$ is essentially, the logarithm of the partition function; which is itself the sum (or integral) over all states of the exponential of the Hamiltonian. It turns out that $Z$ and $F$ encode, in their functional forms, a great deal of information about the system: various quantities, in particular the system's thermodynamic quantities, can be obtained from them, especially by taking their derivatives. For example, in a ferromagnet, the magnetization is the first derivative of the free energy with respect to the applied magnetic field, and the magnetic susceptibility is the second derivative.

Now, broadly speaking: phase transitions involve abrupt changes, in time and-or space, in thermodynamic quantities: for example, think of the change of particle density in a 
solid-liquid, or liquid-gas, transition. Thermodynamics describes these changes as discontinuities in thermodynamic quantities (or their derivatives), and statistical mechanics follows suit. This means that the statistical mechanical description of phase transitions requires non-analyticities of the free energy $F$. But under widely applicable assumptions, the free energy of a system with finitely many constituent particles (or analogously: sites) is an analytic function of the thermodynamic quantities within it. For example, we will soon see that in the Ising model with $N$ sites, the Hamiltonian $H$ is a quadratic polynomial in spin variables (cf. eq. 7.4). This means that the partition function $Z$, which by eq. 7.2 is a sum of exponentials of $-\beta H$, is analytic; and so also is its logarithm, and the free energy. (This and similar arguments about more general forms of the Hamiltonian (or partition function or free energy), are widespread: e.g. Ruelle (1969, p. 108f.), Thompson (1972, p. 79), Le Bellac (1991, p. 9), Lavis and Bell (1999, pp. 72-3)) .

I of course admit that - as my phrases 'broadly speaking' and 'under widely applicable assumptions' indicate - this argument why phase transitions need the thermodynamic limit is not a rigorous theorem. Hence the effort mentioned in Section 7.1.1 to develop a theory of phase transitions in finite systems; and the philosophical debate among Callender et al. mentioned in this Section's preamble. Hence also the historical struggle to recognize the need for infinite systems: both Emch and Liu (2002, p. 394) and Kadanoff (2009, p. 782; 2010, Section 4.4) cite the famous incident of Kramers putting the matter to a vote at a meeting in memory of Van der Waals in 193725

But this argument, although not a rigorous theorem, is very "robust" - and recognized as such by the literature. For example, Kadanoff makes it one of the main themes of his recent discussions, and even dubs it the 'extended singularity theorem' (2010, Sections 2.2, 6.7.1; 2010a, Section 4.1). He also makes it a playful variation on Anderson's slogan that 'more is different' (as I mentioned in footnote 1). Namely, he summarizes it in Section titles like 'more is the same; infinitely more is different' (2009, Section 1.5; 2010, Section $3)$. In any case, for the rest of this paper, I accept the argument.

Taking the thermodynamic limit introduces new mathematical structures. But (as one might expect from my previous examples) the variety of formalisms in statistical mechanics (and indeed, the variety of justifications for taking the limit) means that there is a concomitant variety of new structures that in the limit get revealed. I mention one classical, and one quantum, example.

In Yang-Lee theory (initiated by Yang and Lee 1952), one uses complex generalizations of the partition function and free energy, and then argues that for any $z \in \mathbb{C}$, there can be a phase transition (i.e. a non-analyticity of $F$ or $Z$ ) at $z$ only if there are zeroes of $Z$ arbitrarily close to $z$. For finite $N, Z$ has finitely many zeroes, so that there can be a

\footnotetext{
${ }^{25}$ Of course, since I have not precisely defined 'thermodynamic limit' - let alone 'phase transition'! - the argument could hardly be a rigorous theorem. Ruelle (1969, Sections 2.3-4, 3.3-5) rigorously discusses conditions for the thermodynamic limit; (cf. also Emch (1972, p. 299; 2006, p. 1159) Lavis and Bell (1999a, pp. 116, 260)). Such discussions bring out how in some models, the limit is not just the idea that, keeping the density constant, the number $N$ of molecules or sites tends to infinity: there are also conditions on the limiting behaviour of short-range forces. This means that the models eventually run up against both aspects of my fourth claim, (4:Unreal), i.e. against what Section 2 called 'atomism' and 'cosmology'.
} 
phase transition only at the zeroes themselves: but all of them lie off the real line, and so are not physical. Taking the limit $N \rightarrow \infty$ "breaks" this last argument: there can be a curve of zeroes that intersects the real axis. Indeed, in Yang-Lee theory one goes on to classify phase transitions in terms of the behaviour of the density of zeroes in $\mathbb{C}$ : (cf. Thompson 1972, pp. 85-88; Ruelle 1969, pp. 108-112; Lavis and Bell 1999a, pp. 114, 125-134).

My quantum example concerns Gibbs states and KMS states. This follows on from Section [6]s discussion of superselection: especially Section 6.2.1 on unitarily inequivalent representations of an algebra of quantities, and these representations differing in the value of a global/macroscopic quantity. (For more details, cf. Emch (1972, pp. 213-223; 2006, Section 5.6-7, pp. 1144-1154); Sewell (1986, pp. 73-80; 2002, pp. 113-123); Emch and Liu (2002, pp. 346-357), Liu and Emch (2005, pp. 142-145, 157-161).)

Thus we recall that the Gibbs state of a finite quantum system with Hamiltonian $H$ at inverse temperature $\beta=\frac{1}{k T}$ is given by the density matrix

$$
\rho=\exp (-\beta H) / \operatorname{tr}(\exp (-\beta H))
$$

and represents the (Gibbsian) equilibrium state of the system. (Note the beautiful analogy with eq.s 7.1 and 7.2 .) It is unique (for given $\beta$ ): thereby precluding the representation of two phases of the system at a common temperature - as one would want for a phase transition, 26

So how can we give a quantum description of phase transitions? The algebraic approach to quantum statistical mechanics proposes some states, viz. KMS states, which are defined on infinite quantum systems and which generalize the notion of a Gibbs state in a way that is (a) compelling mathematically, and (b) well-suited to describing phase transitions. A word about each of (a) and (b):-

(a): A mathematical property of Gibbs states (the 'KMS condition') is made into a definition of an equilibrium state that is applicable to both infinite and finite systems: (for the latter it coincides with the Gibbs state at the given temperature). KMS states can be shown to have various stability or robustness properties that makes them very well suited to describe (stable) physical equilibria. (Emch (2006, Section 5.4, pp. 1128-1142) is a excellent survey of these properties. Such a survey brings out how KMS states could themselves form an example of emergent behaviour, in my sense of novel and robust properties!)

(b): The set $K_{\beta}$ of KMS states at a given inverse temperature $\beta$ is in general not a singleton set. Rather, it is convex, with: (i) every element having a unique expression as a mixture of its extremal points; and (ii) its extremal points being well-suited to describe pure thermodynamical phases (mathematically, they are factor states). Taken together, (i) and (ii) suggest that a compelling representative of the state of a system undergoing a phase transition at inverse temperature $\beta$ is a non-extremal $\omega \in K_{\beta}$.

\footnotetext{
${ }^{26}$ This uniqueness also precludes spontaneous symmetry breaking, understood (as usual) as the allowance of distinct equilibria that differ by a dynamical symmetry - e.g. in Section [6.2.1]s scenario, ground states each with all spins aligned, but in different spatial directions. Spontaneous symmetry breaking is (yet another!) important aspect of phase transitions which I cannot here pursue: a fine recent philosophical discussion is Liu and Emch (2005).
} 
So much by way of indicating justifications for taking "the" thermodynamic limit. I turn to discussing the approach to the limit.

7.1.2.B: Approaching the limit:- I will give a classical example of approaching the limit $N \rightarrow \infty$ of infinitely many sites in a lattice: namely, the phase transition (change of magnetization) of a ferromagnet at sub-critical temperatures, as described by the Ising model with $N$ sites (in two or more spatial dimensions). As I mentioned at the start of this Section, this will develop Section 3.3.2 s dissolving of the "mystery" of describing a finite- $N$ system with a model using infinite $N$.

The Ising model postulates that at each of $N$ sites, a classical "spin" variable $\sigma$ (which we think of as defined with respect to some spatial direction) takes the values \pm 1 . To do Gibbsian statistical mechanics, i.e. to apply eq.s 7.1 and 7.2 , we need to define a Hamiltonian and then sum over configurations. The Hamiltonian is chosen to give a simple representation of the ideas that (i) neighbouring spins interact and tend to be aligned (i.e. their having equal values has lower energy) and (ii) the spins are coupled to an external magnetic field which points along the given spatial direction. Thus the Hamiltonian is

$$
H=J \Sigma_{n n} \sigma_{p} \sigma_{q}+J^{\prime} \Sigma \sigma_{p} .
$$

where: the first sum is over all pairs of nearest-neighbour sites, the second sum is over all sites, $J$ (with the dimension energy) is negative to represent that the neighbouring spins "like" to be aligned, and $J^{\prime}$ is given by the magnetic moment times the external magnetic field.

The simplest possible case is the case of $N=1$ ! With only one site, the Hamiltonian becomes

$$
H=J^{\prime} \sigma
$$

so that if we define a dimensionless coupling $h:=-J^{\prime} / k T$, then eq.s 7.1 and 7.2 give

$$
\operatorname{prob}(+1)=e^{h} / z \text { and } \operatorname{prob}(-1)=e^{-h} / z \text {, with } z=e^{h}+e^{-h}=2 \cosh h .
$$

This implies that the magnetization, i.e. the average value of the spin, is

$$
\langle\sigma\rangle=e^{h} / z-e^{-h} / z=\tanh h .
$$

This is as we would hope: the statistical mechanical treatment of a single spin predicts the magnetization increases smoothly from -1 , through zero, to +1 as the applied field along the given axis increases from minus infinity through zero to plus infinity.

What about larger $N$ ? The analytical problem becomes much more complicated (though the magnetization is still a smooth function of the applied field). But the effect is what we would expect: a larger $N$ acts as a brake on the ferromagnet's response to the applied field increasing from negative to positive values (along the given axis). That is: the increased number of nearest neighbours means that the ferromagnet "lingers longer", has "more inertia", before the rising value of the applied field succeeds in flipping the magnetization from -1 to +1 . More precisely: as $N$ increases, most of the change in the magnetization occurs more and more steeply, i.e. occurs in a smaller and smaller interval around the applied field being zero. Thus the magnetic susceptibility, defined as 
the derivative of magnetization with respect to magnetic field, is, in the neighbourhood of 0 , larger for larger $N$, and tends to infinity as $N \rightarrow \infty$. As Kadanoff says: 'at a very large number of sites ... the transition will become so steep that the causal observer might say that it has occurred suddenly. The astute observer will look more closely, see that there is a very steep rise, and perhaps conclude that the discontinuous jump occurs only in the infinite system' (2009, p. 783; and Figure 4; cf. also 2010, p. 20, Figure 5).

Clearly, this example corresponds closely to that in Section 3.3.2 s dissolving of the "mystery". And this general picture of the approach to the $N \rightarrow \infty$ limits applies much more widely. In particular, very similar remarks apply to liquid-gas phase transition, i.e. boiling. There the quantity which becomes infinite in the $N \rightarrow \infty$ limit, i.e. the analogue of the magnetic susceptibility, is the compressibility, defined as the derivative of the density with respect to the pressure. And I am happy to give a hostage to fortune: as I declared in Section 3.3.2 s footnote 9, I believe this example is a good prototype for dissolving the corresponding alleged mystery in physics' other 'singular' limits. Though I cannot argue for that here, I note that the views of some philosophers discussing phase transitions seem to mesh with it: e.g. Mainwood's proposal in Section 7.2.1 below; Bangu's appeal (2009, p. 497f.) to Bogen and Woodward's (1988) distinction between data and phenomena; and Menon and Callender (2011, Sections 3, 4).

\subsection{The claims illustrated by emergent phase transitions}

I will not now devote a Subsection to each of my three main claims, (1:Deduce), (2:Before) and (3:Herring), as I did for my first three examples. It would take too much space - and much more detail that Section 7.1 has supplied - to do so. Thus (1:Deduce) would require me to properly define: (a) a handful of novel and robust behaviours shown in phase transitions (a handful of $T_{t} \mathrm{~s}$ ), and (b) a corresponding handful of statistical mechanical theories $T_{b}$ in which the behaviours are rigorously deducible if one takes an appropriate version of "the" thermodynamic limit, $N \rightarrow \infty$-but not otherwise. Similarly, for (2:Before) and (3:Herring). Doing all that properly would require a Section as long as Section 6 ... and as to (3:Herring), I would anyway prefer not to flog horses that by now should be dead!

Instead, I will just summarize how phase transitions illustrate the three claims, and endorse a proposal of Mainwood's about emergence before the limit: i.e. about how to think of phase transitions in finite- $N$ systems (Section 7.2.1). Then I will briefly report a remarkable class of phenomena associated with phase transitions, viz. crossover phenomena; (Section 7.2.2). These phenomena make emergence before the limit even more vivid than it was in my previous examples; for they show how an emergent phenomenon can be first gained, then lost, as we approach a phase transition. And this will illustrate (4:Unreal) as well as (2:Before). (For (ii), as for (i), I learnt what follows from Paul Mainwood (2006, especially Chapters 3 and 4). So all this Subsection owes a great deal to him.) 


\subsubsection{Emergence in the limit, and before it: Mainwood's proposal}

My main claims are my two reconciling claims, (1:Deduce) and (2:Before). Applied to phase transitions, they would say, roughly speaking:

(1:Deduce): Some of the emergent behaviours shown in phase transitions are, when understood (as in thermodynamics) in terms of non-analyticities, rigorously deducible within a statistical mechanical theory that takes an appropriate version of the $N \rightarrow \infty$ limit. But they are not deducible in a theory that sticks to finite $N$; so that if one concentrates on finite $N$, one will claim irreducibility.

(2:Before): But these behaviours can also be understood more weakly; (no doubt, this is in part a matter of understanding them phenomenologically). And thus understood, they occur before the limit, i.e. in finite- $N$ systems.

Here I admit that the phrases 'some of the emergent', 'appropriate version' and 'can be understood more weakly' are vague in ways which, as I said in the preamble, I have not the space to make precise: hence my saying 'roughly speaking'. But I still submit that the claims are true, for a wide class of emergent behaviours; and that Section 7.1.2 gives good evidence for this. More precisely, Section 7.1.2.A supports (1:Deduce), and Section 7.1.2.B supports (2:Before) 27 By way of summing up: one can check that the six previous morals, (i) to (vi), that were used in Sections 5.4 and 6.4 to sum up the fractals and superselection examples, apply again.

Finally, I would like to briefly report and endorse a proposal of Mainwood's (2006, Section 4.4.1, p. 238; 2006a, Section 4.1) which fits well with the swings-and-roundabouts flavour of my combining (1:Deduce), especially its negative claim of non-deducibility at finite $N$, with (2:Before). Mainwood's topic is, not emergence in general, but the recent philosophical debate about phase transitions in finite systems, especially as focussed by Callender's (2001, p. 549) presentation of four jointly contradictory propositions about phase transitions. Mainwood first gives a very judicious survey of the pros and cons of denying each of the four propositions, and then uses its conclusions to argue for a proposal that evidently reconciles: (a) statistical mechanics' use of the thermodynamic limit to describe phase transitions in terms of non-analyticities; and (b) our saying that phase transitions occur in the finite system. That is, to take a stock example: Mainwood's proposal secures that a kettle of water, though a finite system, can boil!

Mainwood's proposal is attractively simple. It is that for a system with $N$ degrees of freedom, with a free energy $F_{N}$ that has a well-defined thermodynamic limit, $F_{N} \rightarrow F_{\infty}$, we should just say:

phase transitions occur in the finite system iff $F_{\infty}$ has non-analyticities.

And if we wish, we can add requirements that avoid our having to say that small systems (e.g. a lattice of four Ising spins laid out in a square) undergo phase transitions. Namely: we can add to the above right-hand side conditions along the following lines: and if $N$ is large enough, or the gradient of $F_{N}$ is steep enough etc. (Of course, 'large enough' etc.

\footnotetext{
${ }^{27}$ Besides, a theory of phase transitions in finite systems of the kind argued for by Gross (mentioned in Section 7.1.1) would surely illustrate (2:Before), rather than refute (1:Deduce)'s negative claim of nondeducibility at finite $N$. For of course, a theory like Gross' cannot overturn pure mathematical arguments, for example about the analyticity of certain forms of partition function.
} 
are vague words. But Mainwood thinks that the consequent vagueness about whether a phase transition occurs is acceptable; and I agree - after all, 'boil' etc. are vague.)

\subsubsection{Cross-over: gaining and losing emergence at finite $N$}

I end by describing cross-over phenomena. I again follow Mainwood, who uses it (2006, Sections 4.4.2-3, pp. 242-247; 2006a, Section 4.2) to illustrate and defend his proposal for phase transitions in finite systems. I concur with that use of it. But my own aims are rather different. The main idea (mentioned at the start of Section 2 and the end of Section 5.3) will be that cross-over phenomena yield striking illustrations of "oscillations" between (2:Before) and (4:Unreal). That is: a system can be:

(i) first, manipulated so as to illustrate (2:Before), i.e. an emergent behaviour at finite $N$; and

(ii) then manipulated so as to lose this behaviour, i.e. to illustrate (4:Unreal); by the manipulation corresponding to higher, and unrealistic, values of $N$; and

(iii) then manipulated so as to either (a) enter a regime illustrating some other emergent behaviour, or (b) revert to the first emergent behaviour; so that either (a) or (b) illustrate (2:Before) again.

In short: cross-over will illustrate my swings-and-roundabouts combination of (2:Before) and (4:Unreal).

Besides, cross-over will illustrate a simpler point about (2:Before), which we already saw for my first two examples: viz., how the emergent behaviour that one "sees" at large but finite $N$, can be "lost" if one alters certain features of the situation. We saw this:

(a) for the method of arbitrary functions: by raising one's standard of how close to exact equiprobability was "close enough", or by widening the class of density functions with respect to each of which one required approximate equiprobability; (Sections 4.2.1, 4.2.2) and

(b) for fractals: by "getting better eyesight", i.e. by reducing the length-scale on which one could resolve spatial structure - and so see that at the given finite $N$, there was not yet the infinitely descending tower of structure characteristic of a fractal; (Section 5.2.2).

Cross-over occurs near a critical phase transition. This is one where a quantity called the correlation length $\xi$, which summarizes the average length-scale on which microscopic quantities' values are correlated, diverges (in the modest sense of growing without bound, reviewed in Section 3.1). Understanding many such transitions (quantitatively as well as qualitatively), and understanding cross-over in particular, is one of the great successes of the renormalization group $(\mathrm{RG})$ techniques that have been developed over the last fifty years.

Beware: some recent philosophical literature, waxing enthusiastic about the RG, suggests that every phase transition has a "singular" thermodynamic limit and-or infinite correlation length. Not so: witness the fact that until now, this Section has not had to mention the RG! More generally, I reiterate my point (e.g. in the preamble to Section 3 and in Section 6.2.2) that there can be emergent limiting behaviour, with nothing "singular" about the limit. But enough admonition! I turn to describing cross-over; (for details, 
cf. e.g. Yeomans (1992, p. 112), Cardy (1997, pp. 61, 69-72), Chaikin and Lubensky (2000, pp. 216, 270-3); Hadzibabic et al. (2006) is a recent example of experiments).

As substances approach a critical phase transition, they typically show behaviour characteristic of one of a small number of universality classes. Cross-over happens when a substance appears to show behaviour characteristic of one universality class, but then suddenly changes to another as it is brought even closer to its critical point. To explain this, we first recall the basic idea of the $\mathrm{RG}$, as follows.

(1): We define a space $X$ coordinatized by the parameter values that define the microscopic Hamiltonian, e.g. interaction strengths between particles, and the strength of the coupling to an external field; (and typically, also temperature).

(2): We define a transformation $T$ on $X$ designed to preserve the large-scale physics of the system. Typically, $T$ is a coarse-graining, defined by local collective variables that take some sort of majority vote about the local quantities' values, followed by a rescaling, so that the resulting system can be assigned to a point in $X 28$

(3): This assignment of the resulting system to a point within $\mathrm{X}$ enables one to consider iterating $T$, so that we get a flow on $X$. Critical points where $\xi$ diverges will be among the fixed points of this flow. For the fact that $\xi$ diverges means that the system "looks the same" at all length-scales, so that $T$ fixes (makes no change in) the description of the system. (Besides, this scale-invariance can involve power-law behaviour on all scales and self-similarity, and so lead to the use of fractals, e.g. to describe the distribution of sizes of the "islands" of aligned spins in a two- or three-dimensional Ising model; cf. the end of Section 5.3)

I can now describe cross-over. I choose a kind called finite-size cross-over. This occurs when the ratio of $\xi$ to the system's size determines the fixed point towards which the $\mathrm{RG}$ flow sends the system. When $\xi$ is small compared to the size of the system, though very large on a microscopic length-scale, the system flows towards a certain fixed point representing a phase transition; and so exemplifies a certain universality class. Or to put it more prosaically: coarser and coarser (and suitably rescaled) descriptions of the system are more and more like descriptions of a phase transition. So in the jargon of my claims: the system illustrates (2:Before). But as $\xi$ grows even larger, and becomes comparable with the system size, the flow crosses over and moves away - in general, eventually, towards a different fixed point. In my jargon: the system runs up against (4:Unreal), and goes over to another universality class - eventually to another behaviour illustrating (2:Before).

Of course, the correlation length will only approach a system's physical size when the system has been brought very close indeed to the phase transition, well within the usual experimental error. That is: until we enter the cross-over regime, experimental data about quantities such as the gradient of the free energy will strongly suggest non-analyticities, such as a sharp corner or an infinite peak. Or in other words: until we enter this regime, the behaviour will be as if the system is infinite in extent. But once we enter this regime, and the cross-over occurs, the appearance of non-analyticities goes away: peaks become tall and narrow - but finitely high. Again, in my jargon, we have: (2:Before) followed by

\footnotetext{
${ }^{28}$ Hence another playful variation by Kadanoff on Anderson's slogan (cf. footnote 1); Section 6.4 of Kadanoff (2010) is called 'Less is the same'.
} 
(4:Unreal).

Finally, I remark that a similar discussion would apply to other kinds of cross-over, such as dimensional cross-over. For example, this occurs when the behaviour of a thin film crosses over from a universality class typical of three-dimensional systems to one for two-dimensional systems, as $\xi$ becomes comparable with the film's thickness.

\subsection{Envoi}

I believe that my claims, in particular my two main ones, (1:Deduce) and (2:Before), are illustrated by many examples beyond the four I have chosen. For instance, to stick to the area of my main physics example, viz. the $N \rightarrow \infty$ limit of quantum theory: there are Sewell's own examples of his scheme in Section 6.1.1, and KMS states' description of thermodynamic phases (Section 7.1.2,A). Showing my claims in many such examples would indeed be strong testimony to the reconciliation of emergence and reduction. Work for another day!

\section{Acknowledgements:-}

I dedicate this paper to the memory of Peter Lipton, a superb philosopher and a wonderful person. I am indebted to many audiences and colleagues. For invitations to lecture, I am very grateful to: the Royal Society, Bristol University, the Italian Society for Philosophy of Science, Lee Gohlicke and the Seven Pines Symposium, the Lorentz Centre at the University of Leiden, the University of Pittsburgh and Sandra Mitchell, Princeton University, and the organizers of the conferences Frontiers of Fundamental Physics 11, and Emergence in Physics. I am also grateful: to my hosts and audiences at these occasions, and at seminars in Cambridge, Chicago (twice), Maryland, Minnesota, Oxford and Sydney; to A. Caulton, R. Bishop, S. Bangu, E. Curiel, C. Callender, P. Humphreys, O. Shenker, E. Sober and A. Wayne, and especially to Paul Mainwood, Leo Kadanoff and two referees, for comments on previous versions and discussions; and to the editors - not least for their patience.

\section{References}

Avnir, D., Biham O., et al (1998), 'Is the geometry of nature fractal?' Science $\mathbf{2 7 9}$, 39-40.

Anderson, P. (1972), 'More is different', Science 177, pp. 393-396; reprinted in Bedau and Humphreys (2008).

Bangu, S. (2009), 'Understanding thermodynamic singularities: phase transitions, data and phenomena', Philosophy of Science 76, pp. 488-505.

Barnsley, M. (1988), Fractals Everywhere, Academic Press, Boston, 1988.

Barrenblatt, G. (1996), Scaling, Self-similarity and Intermediate Asymptotics, Cam- 
bridge University Press.

Batterman, R. (1992), 'Explanatory instability', Nous 26, pp. 325-348.

Batterman, R. (2002), The Devil in the Details, Oxford University Press.

Batterman, R. (2005), 'Critical phenomena and breaking drops: infinite idealizations in physics', Studies in History and Philosophy of Modern Physics 36B, pp. 225-244.

Batterman, R. (2006), 'Hydrodynamic vs. molecular dynamics: intertheory relations in condensed matters physics', Philosophy of Science 73, pp. 888-904.

Batterman, R. (2009), 'Emergence, singularities, and symmetry breaking', Pittsburgh arXive.

Batterman, R. (2010), 'On the explanatory role of mathematics in empirical science', British Journal for the Philosophy of Science 61, pp. 1-25.

Bedau, M. and Humphreys, P. (eds.) (2008), Emergence: contemporary readings in philosophy and science, MIT Press: Bradford Books.

Belot, G. (2005), 'Whose Devil? Which Details?, Philosophy of Science 72, pp. 128153; a fuller version is available at: http://philsci-archive.pitt.edu/archive/00001515/

Berry, M. (1994), 'Asymptotics, singularities and the reduction of theories', in D. Prawitz, B. Skyrms and D. Westerdahl eds. Logic, Methodology and Philosophy of Science IX: Proceedings of the Ninth International Congress of Logic, Methodology and Philosophy of Science, Uppsala, Sweden 1991, Elsevier Science, pp. 597-607.

Bogen, J. and Woodward, J. (1988), 'Saving the phenomena', Philosophical Review 97, pp. 303-352.

Bouatta, N. and Butterfield, J. (2011), 'Emergence and reduction combined in phase transitions', forthcoming in J. Kouneiher ed. Frontiers of Fundamental Physics 11, (Proceedings of the Conference), American Institute of Physics.

Brady, R. and Ball, R. (1984), 'Fractal growth of copper electrodeposits', Nature 309, Issue 5965, pp. 225-229.

Butterfield, J. (2010), 'Emergence, reduction and supervenience: a varied landscape', in this issue of Foundations of Physics.

Callender, C. (2001), 'Taking thermodynamics too seriously', Studies in History and Philosophy of Modern Physics 32B, pp. 539-554.

Cardy, J. (1997), Scaling and Renormalization in Statistical Physics, Cambridge Lecture Notes in Physics, volume 5; Cambridge University Press.

Castaing, B., Gunaratne, G. et al. (1989), 'Scaling of hard thermal turbulence in Rayleigh-Benard convection', Journal of Fluid Mechanics 204, pp. 1-30.

Cat, J., (1998), 'The physicists' debates on unification in physics at the end of the twentieth century', Historical Studies in the Physical and Biological Sciences, 28 pp. 253300.

Chaikin, P. and Lubensky, T. (2000), Principles of Condensed Matter Physics, Cam- 
bridge University Press.

Colyvan, M. (2005), 'Probability and ecological complexity': a review of Strevens (2003), Biology and Philosophy 20, pp. 869-879.

Diaconis, P. (1977), 'Finite forms of de Finetti's theorem on exchangeability' Synthese 36, pp. 271-281.

Diaconis, P. and Freedman D. (1980), 'De Finetti's generalizations of exchangeability', in R. Jeffrey ed. Studies in Inductive Logic and Probability, volume 2, University of California Press, pp. 233-249.

Diaconis, P. Holmes, S. and Montgomery R.(2007), 'Dynamical bias in the coin toss', SIAM Review 49 pp. 211-235.

Dixon, P., Wu, L. et al. (1990), 'Scaling in the relaxation of supercooled liquids', Physical Review Letters 65, pp. 1108-1111.

Emch, G. (1972), Algebraic Methods in Statistical Mechanics and Quantum Field Theory, John Wiley.

Emch, G. (2006), 'Quantum Statistical Physics', in Philosophy of Physics, Part B, a volume of The Handbook of the Philosophy of Science, ed. J. Butterfield and J. Earman, North Holland, pp. 1075-1182.

Emch, G., and Liu, C. (2002), The Logic of Thermo-statistical Physics, Springer-Verlag

Engel, E. (1992), A Road to Randomness in Physical Systems, Springer.

Falconer, K. (2003), Fractal Geometry, John Wiley.

Frigg, R. and Hoefer, C. (2010), 'Determinism and chance from a Humean perspective', forthcoming in The Present Situation in the Philosophy of Science, edited by D. Dieks, W. Gonzalez, S. Hartmann, F. Stadler, T.Uebel and M. Weber, Springer.

Goldenfeld, N., Martin O. and Oono, Y. (1989), 'Intermediate asymptotics and renormalization group theory', Journal of Scientific Computing 4, pp. 355-372.

Gross, D. (2001), Microcanonocal Thermodynamics; phase transitions in small systems, World Scientific.

Hadzibabic, Z. et al. (2006), 'Berezinskii-Kosterlitz-Thouless crossover in a trapped atomic gas', Nature 441, 29 June 2006, pp. 1118-1121.

Hastings, H. and Sugihara, G. (1993), Fractals: a User's Guide for the Natural Sciences, Oxford University Press.

Hooker, C. (2004), 'Asymptotics, reduction and emergence', British Journal for the Philosophy of Science 55, pp. 435-479.

Jeffrey, R. (1988), 'Conditioning, Kinenatics and Exchangeability', in B. Skyrms and W. Harper ed.s, Causation, Chance and Credence volume 1, pp. 221-255, Kluwer.

Kadanoff, L. (2009), 'More is the same: phase transitions and mean field theories', Journal of Statistical Physics 137, pp. 777-797; available at http://arxiv.org/abs/0906.0653

Kadanoff, L. (2010), 'Theories of matter: infinities and renormalization', forthcoming 
in R. Batterman ed., The Oxford Handbook of the Philosophy of Physics, Oxford University Press; available at http://arxiv.org/abs/1002.2985; and at http://jfi.uchicago.edu/ leop/AboutPapers/Trans2.pdf.

Kadanoff, L. (2010a), 'Relating theories via renormalization', available at http://jfi.uchicago.edu/ leop/AboutPapers/RenormalizationV4.0.pdf

Keller, J. (1986), 'The probability of heads', American Mathematical Monthly 93, pp. 191-197.

Koenig, R. and Renner, R. (2005), 'A de Finetti representation for finite symmetric quantum states', Journal of Mathematical Physics 46, 012105; available at arXiv:quant-ph/0410229v1

Koenig, R. and Mitchison, G. (2007), 'A most compendious and facile quantum de Finetti theorem', Journal of Mathematical Physics 50, 012105; available at arXiv:quant-ph/0703210

Kritzer, P. (2003), Sensitivity and Randomness: the Development of the Theory of Arbitrary Functions, Diplom thesis, University of Salzburg.

Landsman, N. (2006), 'Between Classical and Quantum', in Philosophy of Physics, Part A, a volume of The Handbook of the Philosophy of Science, ed. J. Butterfield and J. Earman, North Holland, pp. 417-554; available at arxiv:quant-ph/0506082 and at http://philsci-archive.pitt.edu/archive/00002328.

Lavis, D. and Bell, G. (1999), Statistical Mechanics of Lattice Systems 1; Closed Forms and Exact Solutions, Springer: second and enlarged edition.

Lavis, D. and Bell, G. (1999a), Statistical Mechanics of Lattice Systems 2; Exact, Series and Renormalization Group Methods, Springer.

Le Bellac, M. (1991), Quantum and Statistical Field Theory, (translated by G. Barton) Oxford University Press.

Liu, C. (2001), 'Infinite systems in SM explanation: thermodynamic limit, renormalization (semi)-groups and ireversibility' Philosophy of Science 68 (Proceedings), pp. S325-S344.

Liu, C. and Emch, G. (2005), 'Explaining quantum spontaneous symmetry breaking', Studies in History and Philosophy of Modern Physics 36, pp. 137-164.

Mainwood, P. (2006), Is More Different? Emergent Properties in Physics, D.Phil. dissertation, Oxford University. At: http://philsci-archive.pitt.edu/8339/

Mainwood, P. (2006a), 'Phase transitions in finite systems', unpublished MS; (corresponds to Chapter 4 of Mainwood (2006). At: http://philsci-archive.pitt.edu/8340/

Mandelbrot, B. (1982), The Fractal Geometry of Nature. San Francisco: Freeman.

Menon, T. and Callender C. (2011), 'Going through a phase: philosophical questions raised by phase transitions', forthcoming in R. Batterman ed. The Oxford Handbook of Philosophy of Physics, Oxford University Press.

Myrvold, W. (2011), 'Deterministic laws and epistemic chances', unpublished manuscript.

Nagel, E. (1961), The Structure of Science: problems in the Logic of Scientific Expla- 
nation, Harcourt.

Nelson, E. (1987), Radicaly Elementary Probability Theory, Princeton University Press, Annals of Mathematics Studies number 117.

Peitgen, H.-O. and Richter P. (1986), The Beauty of Fractals, Springer-Verlag, Heidelberg.

Poincaré, H. (1896), Calcul de Probabilities, Gauthier-Villars, Paris; page reference to 1912 edition.

Richardson, L. (1961), 'The problem of contiguity: an appendix of statistics of deadly quarrels', General Systems Yearbook 6, pp. 139-187.

Renner, R. (2007), 'Symmetry implies independence', Nature Physics 3, pp. 645 - 649.

Rueger, A. (2000), 'Physical emergence, diachronic and synchronic', Synthese 124, pp. 297-322.

Rueger, A. (2006), 'Functional reduction and emergence in the physical sciences', Synthese 151, pp. 335-346.

Ruelle, D. (1969), Statistical Mechanics: Rigourous Results, W.A. Benjamin.

Sewell, G. (1986), Quantum Theory of Collective Phenomena, Oxford University Press.

Sewell, G. (2002), Quantum Mechanics and its Emergent Microphysics, Princeton University Press.

Shenker, O. (1994), 'Fractal Geometry is not the Geometry of Nature', Studies in the History and Philosophy of Science 25, pp. 967-982.

Simon, H. (1996), 'Alternative views of complexity', Chapter 7 of his The Sciences of the Artificial, third edition, MIT Press; the Chapter is reprinted in Bedau and Humphreys (2008); page reference to the reprint.

Smith, P. (1998), Explaining Chaos, Cambridge University Press.

Sober, E. (2010), 'Evolutionary Theory and the Reality of Macro-probabilities', in E. Eells and J. Fetzer ed.s, The Place of Probability in Science (Boston Studies in the Philosophy of Science volume 284), Springer, pp. 133-161.

Stoppard, T. (1993), Arcadia. London: Faber and Faber.

Strevens, M. (2003), Bigger than Chaos: Understanding Complexity through Probability, Harvard University Press.

Thompson, C. (1972), Mathematical Statistical Mechanics, Princeton University Press.

von Plato, J. (1983), 'The method of arbitrary functions', British Journal for the Philosophy of Science 34, pp. 37-47.

von Plato, J. (1994), Creating Modern Probability, Cambridge University Press.

Wayne, A. (2009), 'Emergence and singular limits', forthcoming in Synthese; available at http://philsci-archive.pitt.edu/archive/00004962/.

Weinberg, S. (1987), 'Newtonianism, reductionism and the art of congressional testi- 
mony', Nature 330, pp. 433-437; reprinted in Bedau and Humphreys (2008); page reference to the reprint; also reprinted in Weinberg, S. Facing Up: Science and its Cultural Adversaries, Harvard University Press, pp. 8-25.

Werndl, C. (2010), Review of Strevens (2003), forthcoming in British Journal for the Philosophy of Science.

Wimsatt, W. (1997), 'Aggregativity: reductive heuristics for finding emergence', Philosophy of Science 64, pp. S372-S384; reprinted in Bedau and Humphreys (2008); page reference to the reprint.

Yeomans, J. (1992), Statistical Mechanics of Phase Transitions, Oxford University Press. 